On how we experience ourselves as intentional agents An examination of the role of intentional action in the sense of agency

Samantha Antusch 


\title{
On how we experience ourselves as intentional agents
}

\author{
An examination of the role of intentional action \\ in the sense of agency
}

Hoe wij onszelf als intentionele individuen ervaren

Een studie naar de rol van intentionele actie in het gevoel van "agency"

(met een samenvatting in het Nederlands)

\section{Proefschrift}

ter verkrijging van de graad van doctor aan de Universiteit Utrecht op gezag van de rector magnificus, prof. dr. H.R.B.M. Kummeling, ingevolge het besluit van het college voor promoties

in het openbaar te verdedigen op vrijdag 8 januari 2021 des middags te 12.45 uur

door

\section{Samantha Antusch}

geboren op 26 februari 1992

te Berlijn, Duitsland 
Promotor: $\quad$ Prof. dr. H. Aarts

Copromotor: Dr. R. Custers 


\section{Table of Contents}

Chapter 1 Intentional action and the sense of agency: Introduction and overview

Chapter 2

Intentionality and temporal binding: Do causality beliefs increase the perceived temporal attraction between events?

Chapter 3

Sense of agency in the absence of motor movement: An investigation into temporal binding of tactile sensations and auditory effects

Chapter 4

Intentional action and limitation of personal autonomy. Do restrictions of action selection decrease the sense of agency?

Chapter 5

The role of intentional strength in shaping the sense of agency

Addendum

Supplementary material 149

Contributions

Dutch summary (Nederlandse samenvatting)

References

Acknowledgements 
Chapter 1

Intentional action and the sense of agency: Introduction and Overview 
Whether it is pushing the power-button on the remote control to switch on the TV to watch a movie, opening the fridge to get a chilled soda or turning the car key to start the motor engine - people engage in intentional actions on a daily basis. Intentional actions derive from a personal goal in which an individual represents and controls his actions in terms of their effects. Thus, for example, when a person wants to watch the news, she may form the intention to switch on the TV (effect), which leads to pushing the power-button (action). Although intentional action is a pervasive part of life, we barely ever think much about how it comes about and the consequences it has. At a more general level, intentional action constitutes the basis for judgments of responsibility and moral reasoning. In doing so, intentional action is reflected in the juridical system, which builds on the assumption that humans are able to act intentionally in line with their goals (Haggard, 2017; Haggard \& Tsakiris, 2009). At a more individual level of analysis, intentional actions are associated with distinctive experiences involved in sensorimotor and perceptual processes. Specifically, research on the cognitive neuroscience of volition suggests that intentional action leads to perceptual integration of our actions and their effects and is closely linked to a person's sense of agency, i.e. the intrinsic experiences of being the author of one's own actions and resulting effects in the environment.

The current dissertation addresses this ability of people to consider themselves as intentional agents. The role of intentions in shaping human action and experiences of control is still debated, and empirical evidence is rather mixed. I will present a series of studies that examined the role of intentional action in forming the sense of agency. Specifically, I scrutinized the key aspects of intentions that are thought to cause people to perceptually bind their actions and the resulting effects together, forming the basis for the sense of agency. In this chapter, I will provide a short overview of the field and the theoretical underpinnings. Next, I will identify open questions with regards to the empirical understanding of the relation 
between intentional actions and the sense of agency. I will also integrate my findings in the current literature and will end with a set of conclusions and a general discussion.

\section{What is intentional action?}

Philosophers have long aimed to understand and define the concepts of intentionality and intentional action. Early empiricist philosophers, such as John Locke (1823), John Stuart Mill (1861) and Jeremy Bentham (1789), focused on the ethical and moral value of voluntariness of human actions and maintained that a voluntary act is a movement of the body caused by an act of will. Locke called the act of will a 'volition', whereas Bentham and Mill preferred the term 'intention', but their conception of it was the same. Volition or intention was construed as a kind of conscious choosing or deciding.

At the end of the $19^{\text {th }}$ century, Franz Brentano (1874) introduced the idea of intentionality as a means to distinguish between physical and mental phenomena. In this view, intentionality is the mind's ability to form representations - either behavioral in nature or causal and self-referential. John Searle (1983) distinguished between prior intentions and intentions in action. Prior intentions precede an action and can be distant (e.g., to go running tomorrow morning) while intentions in action are immediately linked to the action (e.g., moving one's feet to run). Elisabeth Pacherie (2006) made a similar distinction about the functionality of intentions. She distinguishes between future-intentions (F-intentions), present-directed intentions (P-intentions) and motor-intentions (M-intentions). F-intentions are similar to Searle's definition of prior intentions in that they are detached from a context and are not directly linked to an immediate action. P-intentions and $\mathrm{M}$-intentions are more similar to Searle's intentions in action as they are both involved in the online control of motor action. Importantly, because M-intentions involve motor representations they are therefore directly responsible for the transformation of intentions into actions. 
Other philosophers have focused on understanding the origin of intentions. Central to this, stands the question whether intentions are the antecedents of human behavior or whether they are retrospectively constructed based on our actions. This problem defines the core of the debate between determinism versus free will (Van Inwagen, 1975). While proponents of a deterministic construal of human action argue that all intentional action is predetermined and is only experienced as intentional in retrospect, opponents of this view defend the idea that human action originates from free will and intentions thus precede action.

In his book “The illusion of conscious will”, Daniel Wegner (2002) describes why, despite us experiencing ourselves as if we willed our actions, intentions are constructed, and this experience of consciously willing an action is distinct from the causation of an action by a person's mind. Rather than understanding intentions as the cause of our behavior, he views them as a means to experience control and responsibility over our actions, he termed this process apparent mental causation. Several recent studies offer evidence for this apparent causation take on intentional actions, showing how the mere thought of intentions shapes the illusory conscious experience of intentionality and control over actions (Aarts et al. 2005; Wegner \& Wheately, 1999).

Daniel Dennett (1993), on the other hand, argues for the existence of free will but claims that the way the concept has been viewed, as the antidote to determinism is not correct. Instead, he argues, both notions can be partially reconciled into a unified concept of free will. To Dennett, free will is our capacity to anticipate our future and apply corrective behavior if desired. That is, the future might be determined but that does not mean that individuals cannot correct it. As an example, he mentions an incoming brick. We can see the incoming brick and can anticipate what will happen if the brick hits us; hence to avoid it, we can duck and escape the brick. In that, he does not reject the idea of determinism completely but he argues that its existence does not preclude free will. 


\section{Where does intentional action start?: The Libet clock experiment}

In 1983, Benjamin Libet and colleagues published a paper that presented a now famous experiment that might settle these two views. Putting the idea of human free will to the test, the experiment aimed to understand if conscious intentions precede voluntary action or whether this is only a retrospectively formed experience. In his experiment, participants were attending to a numbered clock face with a rotating clock hand while they were planning and executing an action (a key press) and their readiness potentials (brain activity in the motor cortex and supplementary motor area, preceding motor actions) were measured (Libet et al., 1983). The task of the participants was to indicate where the clock hand was when they felt the urge to move (W-time). Findings were that, contrary to popular belief, conscious intentions did not precede action initiation but vice versa: on average, the readiness potential rose $350 \mathrm{~ms}$ before the reported conscious urge to act, indicating that motor preparation took place before a conscious intention was experienced.

With the publication of the results a controversial discussion, which still lasts to the current day, concerning the existence of free will and conscious intentions was set in motion. While remaining highly cited, the original Libet clock experiment is often criticized for its conceptualization of free will as well as for the methodology it used (e.g., Haggard \& Eimer, 1999; Klemm, 2010; Roskies, 2011). A major concern is the definition of free will as only pertaining to the ability to voluntarily decide to prepare and execute an action. Brass and colleagues, for example, argue that an intentional action is determined by three ingredients: the what, whether and when component (Brass \& Haggard, 2008). The what and when components correspond to being able to decide which action to execute (what) and at what time to do so (when). The whether component signals the possibility to refrain from executing any action (similar to the idea of a free won't, see Obhi \& Haggard, 2004). Libet himself later 
admitted that having a free will would also entail components of a so-called free won't, that is being able to inhibit an action (Obhi \& Haggard, 2004).

\section{The sense of agency as a window to intentional action}

In the original Libet clock experiment, participants were simply asked to move their index finger without it having any consequences. Thus they executed an action that was not followed by an action-effect (e.g., pressing a key did not cause an effect such as a light or a sound). When considering intentional action in a broader perspective of human goal pursuit, one also needs to take into account how people cause an effect to occur with their action. Therefore, findings of the original Libet clock experiment might be informative in the context of action awareness, but they cannot directly serve the understanding of the role of intentional action in the perceptual experience of being in control, as part of the goal-directed control of human action.

More recent work has addressed the importance of action-effect links in examining intentional action and the associated action experiences in conscious awareness (Haggard et al., 2002b; Wegner, 2002). Executing intentional actions is argued to create coherent representations of action performance and is linked to an experience of being in control of one's actions and effects following them. For example, when we applaud a speech of a colleague, we move our hands toward each other, feel the hands touching each other and hear a noise when they hit each other. While being separated by successive action steps, clapping hands is usually being experienced as a coherent action event that we cause ourselves. This experience is also called the sense of agency (Moore \& Obhi, 2012). Whereas the sense of agency appears to come rather naturally to most people, there are cases in which such experiences are disturbed. Specifically, distortions in the sense of agency are associated with psychopathological disorders such as schizophrenia or autism-spectrum disorders (Haggard et 
al., 2003; Renes et al., 2016; Sperduti et al., 2014; Voss et al., 2010). This highlights the importance of understanding intentional action and its reflections in agentic experiences.

\section{The Comparator Model of the Sense of Agency}

The occurrence of a sense of agency is often argued to be rooted in internal comparative predictive mechanisms. Forward prediction models like the comparator model propose that when an action is executed, an efferent copy of the motor command is dispatched to an internal prediction model (e.g., Blakemore et al., 2000). This efference copy is used by internal prediction processes to anticipate the sensory input following the action. Overlap between predictive and actual sensory input is believed to result in the formation of a coherent representation of the action-effect event, causing action and effect to be bound together in temporal perception (Frith et al., 2000). A mismatch between predicted and actual sensory input is believed to result in the absence of temporal integration of action and effect in perception.

On a sensorimotor level, a match in predicted and actual sensory input is reflected in the attenuated (i.e., weakened) sensory perception of self-caused effects versus externally caused effects. This effect is called sensory attenuation and is thought to be the neurobiological basis for distinguishing between our actions and those of other agents. When executing an action to cause an effect, the sensory input following the action can be anticipated based on the efferent motor copy, causing it to be attenuated in sensory perception. This effect has been demonstrated in several sensory domains. A famous example is the human inability to tickle oneself (Blakemore et al., 2000; Weiskrantz et al., 1971). When moving our hand, we can anticipate the tickle, resulting in the sensory experience to be attenuated and the tickle to be experienced as less ticklish than when someone else would tickle us. The most commonly studied sensory domain concerns auditory effects (Blakemore 
et al., 1999). Here sensory attenuation is demonstrated by a stimulus-related effect on the N1 event-related potential component of the EEG signal, such as the auditory N1 - the timelocked amplitude at $100 \mathrm{~ms}$ after presenting a tone - that diminishes for self-produced (versus externally produced) effects (i.e., smaller N1 amplitudes correspond to more attenuation). Accordingly, a tone that follows a self-executed action versus another faction is perceived as less loud. See Figure 2 for a typical experimental set-up in sensory attenuation research testing the sound intensity attenuation as also indicated by an attenuated N1-amplitude (see Figure 2C).

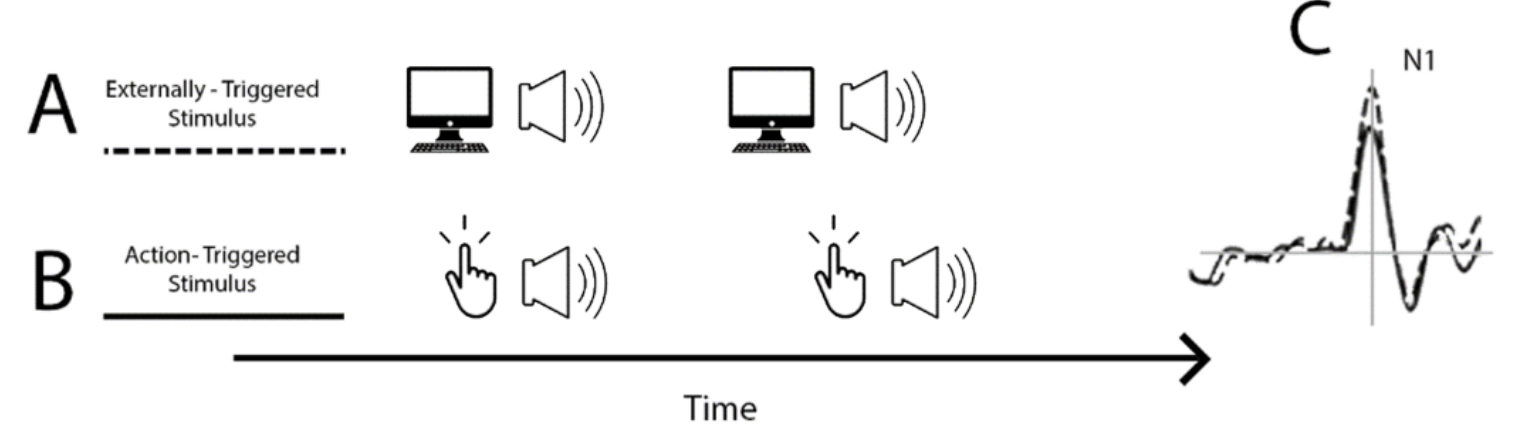

Figure 2. Conditions typically used in sensory attenuation research. In the externally triggered condition (A), a tone triggered by the computer is presented at randomly selected time points. In the action-triggered condition (B), the tone is triggered by self-paced actions performed by the participant. Comparing these conditions typically shows the attenuation of the primary auditory N1 response roughly occurring $100 \mathrm{~ms}$ after the onset of the tone (C). Image adapted based on: Hughes et al. (2013).

While the N1 component of sensory attenuation as detected by neurophysiology is often considered to form the basis for the role of intentional action in agentic experiences, it is unclear how the extrapolation of these models to effects outside of the body would work 
(Dogge et al., 2019). For instance, whereas reduced tickling sensations may result from an efference copy that accompanies sensorimotor processes of self-produced touching, it is less clear how such an efference copy can attenuate perceptual experiences of more externally directed effects, such as pressing a key to switch on a light or sound. It has been suggested that such effects have a stronger basis in perceptual and cognitive processes. In line with this, other methods have been proposed that rely more on perceptual and cognitive processes and might be more informative in the quest to understand the role of intentional action in the sense of agency.

\section{Methods to Assess Sense of Agency}

The sense of agency is often defined and operationalized at two levels of experiences that map well onto two independent processes that are associated with it. One level could be termed the feeling of agency, otherwise often called the implicit or pre-reflective sense of agency, which pertains to the experiences that accompany sensorimotor processes during intentional action. A second level of definition concerns the judgment of agency, which refers to the explicit sense of agency. Judgments of agency are typically obtained with self-report measures such as scales on which participants indicate how much control they experienced over an effect (e.g., "How much control did you have over causing the light to switch on?"). As the psychological output of self-reports and verbalizations relies on accurate access of internal content of the mind, the informative value for the sense of agency is restricted (Dewey \& Knoblich, 2014; Moore et al., 2012). Moreover, people's answers might be prone to biases in memory or motivation that do not necessarily come forth from the processes underlying the sense of agency. Hence, whereas self-reports may reveal how people think and feel about their behavior, this measure might be less suitable for estimating the role of 
intentional action in the sense of agency (Dogge et al., 2014; Renes et al., 2015; Synofzik et al., 2009).

An alternative to assessing the explicit experience of agency is offered by methods that assess the pre-reflective sense of agency, most commonly focusing on the intentional binding effect. This effect occurs when operant actions and their subsequent effects are shifted towards each other in temporal perception, causing the perceptual compression of the temporal interval between them. That is, the action is perceived to have occurred later than it did in actuality while the effect is perceived to have occurred earlier. As this binding effect seems to be absent when an action is induced involuntarily, it has been termed intentional binding (Haggard et al., 2002b). It is also the absence of the effect for involuntary actions that led researchers to consider temporal binding between action and effect as an implicit reflection of sense of agency and read-out of the involvement of intentional action (for a review see Moore \& Obhi, 2012). The intentional binding effect is widely replicated and has been shown to be robust, making it a well-accepted measurement in human agency research. There are two distinct methods for assessing the intentional binding effect, one is the Libet clock method and the other the interval estimation method.

Libet clock method. The Libet clock method is the most commonly used method. In the study that first introduced the method, Haggard and colleagues (2002b) had participants perform operant actions (i.e., key presses that were followed by tones), and used the Libet clock to measure the subjective timing of the actions and their effects. These timings were then compared against a controlled baseline conditions (where the participant only pressed a key without a subsequent tone or only perceived a tone without pressing a key). In the most common set-up of the task, participants complete four randomized blocks (i.e., two baseline and two operant blocks), consisting of several trials each. A trial begins with the presentation of a numbered clock face in the centre of the computer screen and a clock hand, which moves 
at a specific speed (e.g., $2560 \mathrm{~ms}$ per rotation), starting at a random point on the clock face. In baseline trials, participants either only execute an action (key press) or perceive an effect (tone) while attending to the clock face. In operant trials, participants' action is shortly followed by the effect (e.g., $250 \mathrm{~ms})^{1}$. To avoid attention effects, the clock hand rotates further for a random time interval after the last event before it disappears. Subsequently, participants either judge the temporal onset of the action or the effect by indicating where the clock hand was when they executed the action or perceived the tone. Perceptual shifts are calculated based on the difference scores between the baseline and the operant trials. The typically observed pattern is a positive action shift (meaning that the onset of the action was perceived to have occurred closer to the effect) and a negative effect shift (indicative of the onset of the effect tone to have been anticipated), leading to a compression of the temporal interval between action and effect. See Figure 2 for a visualisation of the experimental conditions and the typically occurring perceptual shifts.

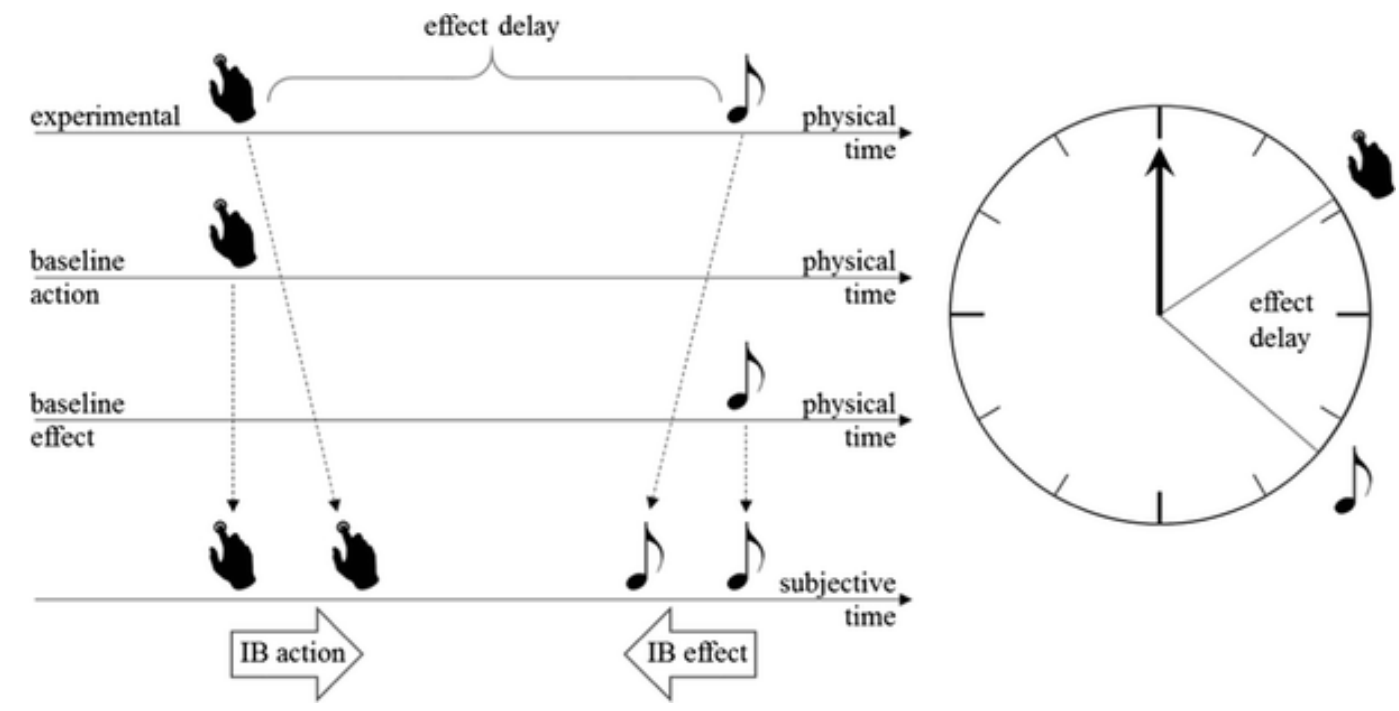

Figure 2. Visualisation of the experimental conditions in the Libet clock task and the perceptual shifts occurring on the action and effect side. Image reprinted with permission

\footnotetext{
${ }^{1}$ Note that the design and size of the Libet clock task has been slightly modified to accommodate limitations of programming software or experimental set-up (such as online research). Importantly, these slight deviations do not alter the typical intentional binding effect (e.g., Cornelio Martinez et al., 2018; Ruess et al., 2018).
} 
from Springer Nature: Attention, Perception, \& Psychophysics, 79(4), Ruess et al., Copyright (2017).

Time interval estimation tasks. Another method to assess intentional binding is offered by time interval estimation or time interval reproduction tasks. In these tasks participants also press a key that is followed by a tone. However, the temporal interval between key and tone is not constant but varies across trials (typically between $100-500 \mathrm{~ms}$ ). Participants' task is then to estimate the length of the temporal interval between action (key press) and effect (tone). While easier to use for participants, this method comes with a range of downsides as well.

One set of issues concerns methodological problems. To assure that a difference in perception can be detected, studies that use this method often have a practice phase in which participants learn to estimate intervals. Participants are typically instructed to use a certain range to estimate the interval (e.g., $0-1000 \mathrm{~ms}$ ). This might cause two primary problems. Firstly, participants might not have a readily accessible concept of an interval of $1000 \mathrm{~ms}$, especially since milliseconds is not a metric used often in daily life. Consequently, some participants do not show differences in perception (i.e., they do not vary in their estimation of the length of various intervals). Secondly, sometimes participants who do not show variation in their estimation of temporal intervals are excluded from the study (Caspar et al., 2016). From Libet clock research, we know that the intentional binding effect is a group-level effect but does not necessarily occur for every individual. Hence, excluding participants who do not show variation in estimating temporal intervals biases the sample and the results.

Besides methodological issues, there are also issues with the interpretation of the results. There seems to be no general consensus as to what constitutes intentional binding when using the interval estimation method, as different studies interpret underestimation of an 
interval as binding, and other studies allow overestimation as evidence for binding ${ }^{2}$ (Damen et al., 2015; Kühn et al., 2013; Wen et al., 2015). Especially overestimation of intervals as evidence for binding is counterintuitive given that binding is defined as a compression of the temporal interval between action and effect. Hence, overestimation would be interpreted as temporal repulsion (i.e., disintegration of the events, causing them to be shifted away from each other in temporal perception).

Libet Clock or Time Interval Estimations? A recent meta-analysis by Tanaka et al. (2019) further indicates several interesting differences between the Libet clock and time interval estimations. Firstly, effects are generally stronger for the Libet clock task than interval estimation procedures. Furthermore, interval estimation does not allow for assessing separate shifts indicating the perceptual change in action awareness and effect anticipation (Tanaka et al., 2019). Assessing the separate shifts reveals an interesting pattern: action awareness shifts are usually significantly smaller than effect awareness shifts. A possible explanation could be that the internal source for the conscious experience of intentional behavior is more focused on the action-effect than on the action itself (see also: van der Weiden et al., 2010). This assessment is not possible when using interval estimation as only the overall compression is captured. This also raises the issue that even when one of the events is actually temporally repulsed (i.e., shifted away from the other event in temporal perception) while the other is temporally bound, interval estimation effects will suggest binding. The Libet clock method is thus much more sensitive and can be used to confirm that the shifts occur indeed bidirectionally, suggesting an actual temporal binding effect.

\section{Causality Perception as an Alternative Explanatory Mechanism}

\footnotetext{
${ }^{2}$ Some authors interpret significant differences in subjectively perceived intervals between conditions as evidence for differences in binding strength.
} 
Opposing forward models are theories claiming that instead of being rooted in intentional processes, temporal binding is the result of causal integration (Buehner \& Humphreys, 2009; 2010). According to such accounts, binding would occur when individuals perceive a causal relationship between action and effect. Because intentional action is associated with causal beliefs about action and effect, such beliefs serve the causal integration of action and effect. Hence, a motor action and associated predicted processes would be superfluous in temporal binding of action and effect and in the sense of agency. This dates back to David Hume (1739/1888) who famously claimed that causation cannot be perceived directly but is inferred. Accordingly, causal inferences from action shape the sense of agency, rather than intentions themselves.

Primary support for the causal integration account comes from studies in which intentional binding was demonstrated in the absence of self-executed actions. Exemplary are studies in which an action is passively induced by a lever or another agent pressing/pulling down the agent's finger to cause a tone (e.g., Borhani et al., 2017; Dogge et al., 2012). The findings are always very similar: binding persists but is considerably decreased when no active motor action and hence no motor prediction is available. These findings are suggestive of another process than motor prediction that results in the integration of action and effect. For example, a possible process, could be causal integration following from beliefs about causal relations between action and effect, binding action and effect together in temporal perceptions. In line with this, a study by Dogge et al. (2012) suggests that such causal beliefs can elevate temporal binding for passively induced actions, likely because agents rely on such beliefs to make sense of why their finger is moved and why the resulting button press causes a tone to sound.

Other evidence comes from studies on action observation, in which an individual does not self-execute an action but observes another agent acting and causing an effect. For 
example, Obhi and Hall (2011) applied a joint-action task in conjunction with the Libet clock paradigm and found that binding strength was indistinguishable for self-initiated versus otherinitiated actions and effects. Further evidence comes from research in which individuals do not act themselves but observe another agent (Poonian \& Cunnington, 2013) or a machine (Buehner, 2012) acting and causing an effect. These findings show that binding persists even when the individual does not execute an action. Moreover, based on these findings, it is proposed that similar neural networks are active when perceiving another person acting and experiencing the consequences of the action (Poonian \& Cunnington, 2013). That would mean that the perceptual integration of observed actions stems from similar prediction processes as are involved in integrating self-executed actions and effects but that those predictions are independent of motor action.

In line with this, Moore et al. (2013) who applied an interval estimation task found evidence for stronger binding for the observation of actions believed to be intentional versus actions believed to be non-intentional. The authors also presented indirect support from fMRI data, suggesting that the sensorimotor system might be covertly activated during observation of an intentional causal action. They concluded that intentional binding is not self-specific and might be due to intentional causation processes rather than motor predictive processes.

\section{Unresolved issues in existing research}

Early research investigating the question of intention versus causality, in shaping perceptual integration, mainly focused on the relationship between action and effect. However, existing research often suffers from motor action confounds, not allowing for the influence of motor action or motor prediction to be ruled out entirely. For example, in studies in which an action is induced by pushing/pulling down a finger, participants could have still started up their motor system once they noticed the movement. The resulting slightly delayed 
motor prediction processes could have contributed to an agentic experience, hence explaining why the intentional binding emerged but was attenuated in magnitude. In some of these studies, participants were also subjected to conditions in which they executed an action and caused an effect (e.g., Borhani et al., 2017; Poonian \& Cunnington, 2013). This action experience could have led to carry-over effects, as participants might have used it to also imagine acting and causing the effect in the observational condition, facilitating intentionality experiences and giving rise to binding effects (for a model that links perception and action, see e.g., James, 1890; Prinz, 1997; Decety \& Grèzes, 2006). Hence, a good empirical test of causal integration as an alternative explanatory mechanism for temporal binding is still lacking.

One potential route to develop such a test is to consider intentional action in a broader context of human action, and to distinguish the different aspects that might be the origin of the temporal integration of action and effect and ultimately the formation of the sense of agency. Intentional actions are commonly considered to be motivated and directed by goals (Deci \& Ryan, 1985; Fishbein \& Ajzen, 1975). Furthermore, intentional action often involves manual action contexts, where people interact with an interface that enables or forces them to press a key to produce an effect. Accordingly, considering intentional action in the context of goaldirected manual actions allows us to distinguish four key-aspects that form targets for examining the role of intentional action in the sense of agency: (a) the sound of pressing a key (a click), i.e. auditory input, (b) the tactile experience of the key press, i.e. action-sensation input, (c) self-chosen or forced action, i.e. fully autonomous or no autonomous action selection, and (d) the degree of motivation to engage in intentional action, i.e. intentional strength. This offers two different starting points for the investigation of intentions in the sense of agency and intentional binding in specific: 
1. Taking all action away from the task and use the (a) auditory input or (b) actionsensation input as a predictor of the 'action'-effect. In this setting, it is also possible to test causal integration and predictive accounts.

2. Focusing on the importance of intentionality itself, especially the process of (c) undermining and (d) amplifying intentions.

Below I will present a more elaborate analysis of each of these aspects, including the studies that we conducted in our laboratory to further address the evidence of these aspects in the study of intentional action and the sense of agency.

\section{Sensory auditory input and the sense of agency}

Humans form coherent representations of their own behavior (Aarts \& Custers, 2009; Vallacher \& Wegner, 1985). In terms of intentional action, self-produced actions that result in sensory feedback, such as triggering a sound or light by pressing keys, are perceived as a coherent event. Whereas such coherency experiences might be action-based, it has been argued that coherency experiences also occur for complex visual scenes and auditory events that are otherwise disconnected in time or space (Serences \& Yantis, 2006; Shamma et al., 2011). Such coherency experiences could also account for the compression of the temporal interval in the binding effect.

Accordingly, an empirical test of the role of intentional action in the sense of agency could be offered by sensory-based non-motor tasks that measure temporal binding with the Libet clock. Such an empirical set-up, that rules out motor confounds (i.e., eliminating any influence of motor behavior and motor predictive processes) also allows to empirically test intention-based versus causal cognition-based theoretical accounts against each other. Instead of testing how action-effect perceptions change under varying conditions, non-motor tasks 
test the perception between external events that were not caused by intentional motor action (Desantis et al., 2012; Haggard et al., 2002a). By removing all motor action from the task, changes in temporal perception of external events can be unequivocally attributed to the manipulated factors (e.g., beliefs about the causal relationship between events).

In an initial study using a non-motor task by Haggard and colleagues (2002a), participants experienced two identical successive auditory stimuli that were separated by a $250 \mathrm{~ms}$ time interval and judged their temporal onsets using the Libet clock. Instead of being temporally bound in perception, the authors found temporal repulsion of the tones, suggesting that the two stimuli were experienced as two separate events. Support for this conclusion also comes from a similar study by Desantis and colleagues (2012) who found that two auditory stimuli, separated by $400 \mathrm{~ms}$, were perceived as temporally repulsed from each other as compared to an action-and-effect condition.

While suggesting the emergence of temporal repulsion, these studies did not statistically test the repulsion effect. Thus it is not clear whether auditory stimuli were indeed repulsed from each other or were simply not bound to each other. Additionally, methodological differences might have led to the disintegration of the tones, causing temporal repulsion instead of binding. For example, in the study by Desantis et al. (2012), an interstimulus interval of $400 \mathrm{~ms}$ was used, which differs from the conventional $250 \mathrm{~ms}$ interval. Moreover, the tone combinations were either congruent or incongruent (with a prior learned predictive association between tones), obstructing the formation of a stable predictive link and leading to participants being unable to anticipate the identity of the (second) tone. However, especially when an effect is not preceded by an intentional action, such a stable predictive link might be necessary to integrate the stimuli and perceive coherency.

A recent study by Antusch et al. (2020, see Chapter 2) elaborated on these early findings and tested how external auditory stimuli are perceived in time when motor action is 
absent. In two studies using a stimulus-based Libet clock task, participants perceived successive auditory stimuli, separated by $250 \mathrm{~ms}$, and judged their temporal onset. Additionally, participants' causal belief was manipulated. While participants in the control condition were not instructed about the relationship between the stimuli, participants in the causal belief condition were explicitly told that the stimuli were causally related. Findings showed and tested a robust temporal repulsion effect, suggesting no perceptual coherence between the two auditory events. Causal belief could attenuate temporal repulsion partially but this effect was fragile, even when participants were extensively trained to causally link the two stimuli to each other.

In short, these results support earlier findings demonstrating that two successive auditory stimuli do not bind together in temporal perception, even though beliefs about their causal relationship are strong. Instead they are repulsed, suggesting that causal beliefs do not drive temporal binding of successive stimuli events.

\section{Sensory Action-Input and the Sense of Agency}

A possible reason why the auditory stimuli in the aforementioned studies were not perceptually integrated could be their modality. In all studies, auditory stimuli were used which might cause disintegration rather than integration as the stimuli enter the sensory system in succession, whereas multisensory stimuli might be more readily integrated as they stem from different sensory sources (Soto-Faraco et al., 2004). Of special interest for understanding the role of intentional actions is tactile action-input. Tactile input occupies a special role in human sensory processing, and is important for making meaningful connections with the social and physical environment (Katz, 2013; Löken \& Olausson, 2010). When engaging in an action, besides being able to predict the identity and onset of the action and its effect, the agent also experiences sensory input. This sensory input stems from various sources. That is, when acting such as when pressing a key to cause a tone, this action does not 
only result in sensory input in the form of the action-effect (e.g., the tone) but is also accompanied by action-related sensory input. In this example, the key press would be accompanied by a distinct tactile sensation occurring when the finger touches the key surface.

So far, tactile sensory input associated with action has not been directly considered as a contributing factor in temporal binding and the sense of agency. A recent study by Antusch et al. ( $a$, under review; see also Chapter 3 ) however shed light on this issue, investigating if sensory action-input and a subsequent auditory tone that is caused by it, are bound together in temporal perception. Furthermore, it was tested whether being able to predict the temporal onset and the identity of the input (i.e., what kind of tactile sensation would occur and when) - two key components of intentional action control - facilitates temporal binding. A robust temporal repulsion effect was established, suggesting that multisensory stimuli with a stable predictive link are not readily integrated and bound together in temporal perception.

Interestingly, this repulsion effect was attenuated when participants could predict the temporal onset and the identity of the tactile sensation. That is, sensory action input was not sufficient to cause the two events to be perceived as coherent in temporal perception, and predictability benefitted coherence perception, but it did not reverse temporal repulsion into binding.

In another study on the role of tactile action-based input, Suzuki et al. (2019) asked participants to observe an artificial hand pressing a button and causing a tone while experiencing simultaneous tactile feedback. Using a time interval estimation task, their findings showed that participants experienced binding in the absence of motor action in such contexts. This suggests that tactile feedback helped participants to view action and effect as related and to integrate them in perception. However, this study also suffered from flaws, which might have confounded the results. Based on the visual input of the moving hand, participants could anticipate the onset of the sensory tactile action-based input. This ability to predict the sensory input and its temporal onset might have caused the integration of the 
stimuli in temporal perception. Moreover, because participants were free to move their own hand, viewing the virtual hand move might have (albeit overtly or covertly) triggered the action in the observer and installed an experience of intentionality, causing them to experience agency over the action and its effect. Temporal binding in such a context could involve similar processes that underlie normal intentional action, instead of being entirely due to the sensory input.

\section{Interim summary: non-motor action involvement and the sense of agency}

So far, we have examined whether causal beliefs are central to temporal binding between action and effect, and whether integration of cause and effect can explain the relation between intentional action and the sense of agency. We have explored this issue by completely taking away action from the task, and only examining binding between two (causally) related sensory events in a non-motor task. We discovered that auditory and tactile sensation that accompany manual actions in the service of goal-directed behavior do not bind together in temporal perception. This suggests neither sensory input alone nor causal beliefs suffice to shape temporal binding and the sense of agency, and that some form of motor movement is needed for the emergence of binding. It is then the question how intentions contribute to binding effects and the experience of agency. To answer this question, we will look at two empirical possibilities to investigate the role of intentions: undermining intentions and strengthening intentions. We will begin by examining the effect of undermining intentions on the sense of agency and will then continue with a review of the empirical evidence regarding the influence of strengthening intentions.

\section{Personal Autonomy: Undermining intentions and the Sense of Agency}


An important part of acting intentionally, is the ability to select and time the onset of one's actions. Taking this ability to choose away from the agent is likely to result in the agent to feel a threat to his personal autonomy. Restrictions in personal autonomy in return are assumed to undermine a person's intention for action, and hence, thwart the experience of agency (Caspar et al., 2016; 2017). Quite some literature addresses this relation between personal autonomy and agency as a vital part of human action (Koestner, 2008; Ryan \& Deci, 2006) but whereas there is some work showing that autonomy is associated with self-reported experienced control (judgment of agency; Schwarz et al., 2019), there is no systematic research done so far testing whether restrictions of personal autonomy weakens intentional binding.

Past research used various manipulations to undermine personal autonomy and also the terminology differed across studies, with some investigating 'freedom of choice' and others the influence of 'restricted actions'. All those studies however tapped into the same broader concept of personal autonomy. A first study by Wenke and colleagues (2009) investigated how the external versus interval action selection affects intentional binding. They showed that there was no difference in binding magnitude for the two types of actions, suggesting no effect of undermining personal autonomy on the sense of agency. However, in the externally instructed action timing condition participants were still able to freely time their action within a given action window, making the action not entirely externally determined.

Barlas and Obhi (2013) adopted a different approach. In their study, the choice alternatives from which an agent could select an action were manipulated. Participants pressed one key, choosing from seven (high level of choice), three (medium level of choice) or one action alternative (no choice). The key press was always followed by the same neutral effect tone. Findings showed stronger temporal integration when participants could choose from a high number of action alternatives (i.e., seven keys) as compared to either a medium 
level (i.e., three keys) or no choice alternatives. The authors took the findings as evidence for more freedom in choice leading to stronger agentic experiences. Nonetheless, these findings proved difficult to replicate in later studies (Tanaka \& Kawabata, 2019). Furthermore, the study by Barlas and Obhi (2013) showed a notable effect, namely in the one key alternative option no intentional binding was found. This is peculiar given that participants usually only press one key in the conventional set-up of the Libet clock task, leading to robust binding effects. Hence one would expect binding to also emerge in the one key (no choice) condition in the experiment by Barlas et al. (2013).

A recent study by Schwarz and colleagues (2019) also used the Libet clock task. In their experiment, participants either executed a free action (i.e., they could choose which of two keys to press) or had to execute an instructed action (i.e., the key they had to press was predetermined). The findings showed no differences in binding magnitude for instructed versus free actions, being indicative of implicit reflections of agentic experiences being unaffected when intentionality is undermined. Pointing at a functional difference between implicit and explicit markers of agency though, participants reported significantly lower experiences of control when an action was instructed as compared to free (cf. Synofzik et al., 2008).

Recent research conducted in our lab corroborates this conclusion (Antusch et al., $b$, under review, see Chapter 4). Expanding the work of Schwarz and colleagues (2019), additionally to restricting personal autonomy over selecting an action, we also manipulated autonomy over timing the action by using a cue-based action trigger. Specifically, action timing was strongly restricted by a forced key press in response to a randomly presented action-cue. In several experiments, we tested how having full autonomy (free action selection and free action timing), partial autonomy (forced action selection and free action timing) and having no autonomy (forced action selection and forced action timing) affects the feeling of 
agency as indexed by the intentional binding effect. Note, that the partial autonomy condition equaled the default condition in intentional binding research in which the participant can only press one key but can freely time when to press it. Critically, we found that all conditions resulted in similarly strong binding effect, with no differences between the conditions. Interestingly though, manipulations did affect the psychological experience of autonomy, with participants reporting higher experienced autonomy in the full autonomy condition as compared to the partial and no autonomy conditions. Thus whereas conscious experiences of autonomy are affected when undermining intentionality by restricting an agent's personal autonomy in selecting and timing an action, it does not influence the sense of agency. Because the sense of agency is suggested to arise from sensorimotor processes, it seems that endogenously starting up one's motor system to act (regardless of whether the action is predetermined of freely selected), is sufficient for binding to evolve.

\section{Intentional Strength and Sense of Agency}

The observation that undermining intentionality by personal autonomy restrictions does not diminish the sense of agency is remarkable, given the often claimed importance of autonomy in feeling like an intentional agent. If undermining intentionality does not affect binding, one may ask if strengthening intentionality would affect it. Established models in social psychology put forward this idea, stipulating that intentions to engage in an action vary in strength, depending on the expected value or importance of performing the behavior (Aarts et al., 2012; Ajzen \& Fishbein, 1977; Sheeran, 2002). Strong intentions facilitate action initiation, as they are more readily implemented and are associated with more effort exertion in the case of encountering obstacles during action execution (Brandstätter et al., 2001; Gollwitzer, 1993; 1999). Based on this, it is probable that also one's sense of agency is 
amplified when executing an action with stronger intentions. We refer to these differences in the motivational nature of intentions as intentional strength.

Initial studies primarily examined the role of motivational factors by manipulating the valence of the action-outcome by manipulating whether the effect following the agent's action was negative, neutral or positive in valence. The findings of these studies are rather mixed. In one study, for example, an agent's action was followed by a monetary gain or a monetary loss (Takahata et al., 2012). In another study, the action-outcomes had a positive (e.g., sounds of happiness) or negative (e.g., sounds of disgust) emotional valence (Yoshie \& Haggard, 2013). Findings from those studies suggest that binding decreases for negative outcomes as compared to positive outcomes. However, some studies did not find differences in binding and agency for emotional valence or even found the opposite pattern of results (Moreton et al., 2017; Moretto et al., 2011; Ruys \& Aarts, 2012). Instead of a decrease in intentional binding, research by Moretto et al. (2011) demonstrated an increase in intentional binding for severely compared to moderately negative (moral) outcomes. Whereas these diverse results are difficult to explain by a single motivation account, the main issue with these studies is that the motivation is implemented in the outcome of action, which confounds predictive (anticipation) and postdictive (inference) processes in affecting intentional binding (see Moore \& Haggard, 2008). More importantly, these studies leave open whether motivational factors that directly target the action, i.e. the intentional strength with which an action is executed), affect binding.

To test how intentional strength affects binding, instead of manipulating the valence of the action-outcome, one needs to focus on the motivational nature of the action itself. To do so, the incentive needs to be independent of the action-effect and should only be linked to the action. A recent study from our lab aimed to test this. In this study, participants learned to press one of two keys (Antusch et al., 2019, see Chapter 5). Which key they had to press was 
determined at the beginning of a trial. Each key had a different reward value (low vs. high). Whether participants earned the reward was entirely dependent on the correct execution of the action. This manipulation induced action intentions of different strength (i.e., stronger intentions when pressing the key with the high reward value). Because the reward value was known at the beginning of the trial and exclusively dependent on the action, postdictive modulation of binding was ruled out. This is a major difference to past research in which effect-valences were manipulated, making it impossible to tease apart the influence of intentional strength and postdictive modulation. The findings showed that actions that were executed with stronger intentions indeed resulted in stronger intentional binding. Intentional strength thus seems to not only be important for steering behavior and facilitating action initiation, but also affects the magnitude of the sense of agency.

\section{Discussion and Conclusion}

In the current chapter, I examined how people consider themselves as intentional agents, and addressed the key question of whether intentional action is related to the sense of agency. The role of intention in action and the sense of agency is debated, and research so far favors two different views: the sense of agency as arising from (1) intentional action as represented in intentional binding between action and effect or (2) causal beliefs and inferences that support the integration of action and effect. To examine the validity of these accounts, I located intentional action in a broader context of human goal pursuit and conducted a series of studies that allowed me to empirically investigate core aspects of intentional action separately. Below I will briefly summarize the general findings, draw conclusions on their basis and discuss the findings in the context of past and current research on the role of intentions in shaping experiences of personal causation and control. In addition, I will highlight the importance of the present findings for applied research. 
In the first set of studies, using a sensory-based non-motor task, we examined whether two auditory stimuli that are related to each other are integrated in temporal perception. Testing temporal binding in a sensory-based non-motor task has the advantage of being able to strictly rule out any motor confounds, and hence intentional action processes. Ruling out any influence of motor prediction, we found that such temporal binding between two related auditory stimuli did not occur, but that they were instead repulsed from each other. These findings actually suggest that participants processed the two stimuli as two separate events, which thus did not allow them to build a coherent representation of the full event (Antusch et al., 2020). These findings are in line with earlier research, which suggested but did not test temporal repulsion between external auditory stimuli (Desantis et al., 2012; Haggard et al., 2002a).

In the study from our lab, we also tested the causal integration account and whether holding a causal belief about the relationship between the stimuli facilitates binding. Findings revealed that while holding a belief about the causal relationship between two stimuli partially decreased temporal repulsion, this effect was fragile and did not cause repulsion to be reversed into binding. Temporal binding is thus unlikely to occur due to causal integration. Interestingly, there is some evidence from past research using different methods than the Libet clock task, suggesting integration of stimuli in causal contexts (Buehner \& Humphreys, 2010; Thanopoulos et al., 2018). One study for example, found evidence for temporal integration of events that are linked by a naturalistic causal association (e.g., a clap on a wooden plank and the resulting sound, Thanopoulos et al., 2018). It is important to note that our study used arbitrary selected auditory stimuli that were not naturally and causally related to each other. Therefore, in comparison to newly learned causal relations between events, it is conceivable that events that have a naturalistic or overlearned causal link might be more 
readily bound together in a coherent perception. This is however purely speculative and requires further research. Based on our studies we can draw the following specific conclusion:

\section{Conclusion 1: Two auditory stimuli that are (believed to be) causally related do not bind in temporal perception. In fact, they repulse from each other in temporal perception.}

Whereas external auditory stimuli are not readily bound together in temporal perception, this might be different for sensory input that accompanies motor action itself. In elaboration of this notion, we looked at research that examined the use of action-related tactile information in the temporal integration of two events. Tactile information is important in exploring and interacting with the external world, and constitutes part of the natural sensory input that an agent experiences when executing an intentional motor action.

Initial findings by a study using a virtual hand set-up in conjunction with the Libet clock task were promising. In a study by Suzuki and colleagues (2019), participants viewed a virtual hand moving and pressing a key to cause a tone, while they received parallel tactile feedback on their index finger. Findings showed that temporal binding occurred in these conditions, despite motor action being absent. When testing the role of tactile feedback in a stimulus-based paradigm, studies conducted in our lab however did not find evidence for temporal binding but a strong temporal repulsion effect (Antusch et al., $a$, under review; see Chapter 3). The effect was slightly weakened when the identity and the temporal onset of the sensory input was predictable but was not reversed into temporal binding.

These opposing findings might be due to difference in cognitive processes. Suzuki and colleagues (2019) provided their participants with visual input that allowed them to view a moving hand. This might have installed an experience of acting and giving rise to the formation of an intention in the agent, thereby resulting in temporal binding even in the 
absence of motor action (Deschrijver et al., 2016; Maselli et al., 2016). Hence, the mere sensations of causally related tactile input and auditory stimuli do not bound to each other in temporal perception. It is likely that tactile sensory input needs to be accompanied by intentionality for temporal binding to arise (Zhao et al., 2016). Based on our findings, we can draw the following specific conclusion:

Conclusion 2: Action-based tactile sensations and causally related auditory stimuli that are predictable in terms of identity and temporal onset do not bind together in temporal perception. Instead, they are repulsed from each other in temporal perception.

So far, we have seen that, in a non-motor task, causal relations between sensory stimuli pertaining to the same modality (auditory) or different modalities (tactile and auditory) are not readily integrated in temporal perception. Moreover, higher order beliefs about the causal relationship and cognitive information regarding predictability of the stimuli could only partially weaken this repulsion. Together, these findings suggest that temporal binding as an index of the sense of agency might require motor action. However, we do not know exactly whether the role of motor action in temporal binding is conditional on the action intention of the agent, and hence, whether temporal binding thus is a unique phenomenon related to intentional action experiences.

Capitalizing on this, we continued with an exploration of the exact role of intentionality in the binding effect. Intentionality is a core component of many social psychological models which, assume that intentions are central to achieving one's goals and enact behavior (Aarts et al., 2012; Ajzen \& Fishbein, 1977; Sheeran, 2002). To understand the benefit of intentions in intentional action experiences, we first looked at studies undermining intentionality by restricting an agent's personal autonomy. 
Specifically, we examined the influence of restricting autonomy over two core aspects of intentional action: action selection (what action to execute) and action timing (when to execute the action). Opposite to what would be expected, the findings of the studies pointed towards a persistence of the pre-reflective sense of agency when action selection and timing were externally determined (Antusch et al., $b$, under review; Schwarz et al., 2019; Wenke et al., 2009). Even when agents could not select what action to execute and when to execute it, binding scores were similar to when they could freely decide about both of these aspects. Interestingly, in contrary to the feeling of agency, the judgment of agency, that is, the explicit experience of autonomy, considerably decreased when agents faced increasing restrictions in personal autonomy. This suggests that judgment of agency and the sense of agency are different aspects of the process underlying experiences of being an intentional agent (Moore et al., 2012; Saito et al., 2015). Whereas judgments of agency rely on verbal reports and require access to the content of the mind, the sense of agency is argued to derive from the sensorimotor processes that result from intentional action. It seems, then, that restrictions in personal autonomy do not render these two aspects to converge, suggesting that the intentional binding might operate independently from the way the mind produces the experience of autonomy and control. Based on findings about the relation between our studies about personal autonomy and the sense of agency we can conclude:

\section{Conclusion 3: Restricting personal autonomy, i.e., being unable to choose how and when to} act, does not weaken intentional binding.

Another way to address whether intention plays a key role in the sense of agency is to examine how the motivational force of an intention affects intentional binding between action and effect. Confirming the idea that the amplification in intentional strength is associated with 
a boost in the sense of agency (Aarts et al., 2012), findings from a study in our lab demonstrated that when the intention with which an action is executed is more rewarding, intentional binding is also intensified. Instead of being linked to the effect of the action, the motivational value in this study was entirely dependent on the execution of the action. This is an important addition to past research that had already demonstrated that the binding effect can be modulated by the valence of action-outcomes but did not test the effect of the motivational value of intentions (e.g., Moretto et al., 2011; Takahata et al., 2012). Thus, we could demonstrate that stronger action intentions are associated with stronger intentional binding and thus an increased sense of agency, leading the following specific conclusion:

\section{Conclusion 4: Intentional strength amplifies the intentional binding effect.}

Based on the four specific conclusions, we can draw two more general conclusions. Firstly, motor action seems to be essential for temporal binding to occur. Neither auditory input nor tactile sensory input is sufficient to cause temporal binding when no motor action is present. However, causal perception and predictability might facilitate partial temporal integration but do not cause actual binding. Given the findings of the reviewed literature and our own findings, an explanation for intentional binding based purely on causal integration seems therefore not very probable. More likely, intentional binding is actually closely linked to the sense of agency.

Secondly, undermining intentionality by restricting personal autonomy over action selection and timing as imposed by the environment does not affect the strength of the binding effect. These findings indicate that endogenously initiating one's own motor action to cause an effect might be sufficient for temporal integration to occur. Indeed, conditions that bypass such self-induced actions, such as direct motor activation by transcranial-magnetic stimulation 
(Haggard et al., 2002b), might very well undermine the binding effect. Moreover, when actions are initiated and executed with more strength, this causes the intentional binding effect to increase as well.

\section{Future directions}

The present dissertation aimed to address the question of how we experience ourselves to be intentional agents. The metacognitive experiences of being an agent can be separated from the sense of agency evolving from sensorimotor processes as is reflected in the intentional binding effect. I demonstrated that motor action is required to perceive coherence between cause and effect and that temporal binding between action and effect is associated with intentional action. In other words, the brain seems to produce a sense of agency when one's own intended actions are experienced as a unit.

Agency is commonly assumed to be key to goal-directed behavior and self-regulation (Dijksterhuis \& Aarts, 2012). For example, agency is critical for setting goals (Locke \& Latham, 1990), planning and implementing instrumental actions (Gollwitzer, 1990), making subjective assessment of controlling one's own actions (Aarts et al., 2005), and motivating people to guide or change one's own behavior (Bandura, 1977; Damen et al., 2015; Karsh et al., 2016). Whereas the link between agency, self-regulation and behavioral change seems obvious, there is not much research that has sought to empirically link the sense of agency to behavioral change. Below I will discuss the present findings in the context of common practices in social and behavioral sciences to bring about behavior change (e.g., Tombor \& Michie, 2017; Tummers, 2019). I will make a clear distinction between the sense of agency as a coherence experience of the self as performing actions (i.e., the minimal self, cf. Gallagher, 
2000) and the metacognitive beliefs that we have about controlling behavior (i.e., the narrative self, cf. Gallagher, 2000).

\section{Sense of agency and Behavioral change: targeting the determinants of behavior}

Behavioral change is commonly addressed in an institutional context by targeting the determinants of behavior. Generally, there are three pathways to achieve this:

(a) Education to instruct and persuade humans on how to act in a desired way. This pathway addresses knowledge, beliefs and skills that people hold to support their behavior.

(b) Incentivization to reward behavior, addressing people's willingness to learn and to optimize behavior.

(c) Environmental adaptation to change the context in which humans act. This pathway targets determinants of behavior that are strongly associated with the environment, such as habits and temptations.

\section{Education}

A popular route to achieve behavioral change is appealing to individuals' cognitions by educating them about the pros and cons of existing behavior and the prevailing social norms. Education can equally be aimed at making individuals aware of their skills or challenging their beliefs and convictions. For example, politicians or policy-makers may urge citizens to act responsibly by smoking less, separating waste and recycling, or by reducing air travel. The underlying idea is that the more knowledgeable an individual is, the more likely he is to be convinced to change his intentions and behavior. As it is cognition-based, this route to behavioral change predominantly targets the narrative self. It is primarily effective for 
improving an agent's self-knowledge and installing self-efficacy beliefs in the agent (Bandura, 1977).

As a result, the agent's confidence in being able to perform the desired behavior may strengthen the explicit beliefs of being in control (judgment of agency). On the downside, and in order to be effective, education and cognition-based strategies require the individual to translate his beliefs into intentional action. Such translation relies on intentions in action (Searle, 1983; Pacherie, 2006), meaning that if an individual's intentions are not activated in close temporal proximity to the moment at which action is required, the educational pathway fails to achieve behavioral change (Fishbein \& Ajzen, 1975; Gollwitzer, 1990) Moreover, the present research suggests that the sensorimotor experiences of intentional action and agency remain unaffected by education and appeals to higher-order cognitions (cf. Chapter 2). Whereas having an explicit experience of agency can be beneficial in perceiving oneself as an intentional agent, this judgment can be flawed and is not necessarily rooted in perceptual processes of intentional action and agency (Dewey \& Knoblich, 2014; Moore et al., 2012). Especially when encountering obstacles or external restrictions in the way of achieving one's goals, the judgment of agency is malleable and can be negatively affected (Schwarz et al., 2019), undermining behavioral change. As the sense of agency (the minimal self) is more stable in the face of such obstructions, pathways targeting it are more successful at achieving lasting behavioral change. It might therefore be fruitful for future research to take into account how the sense of agency and judgment of agency can divert, and to examine under which conditions the sensorimotor experiences of agency and metacognitive views of agency predict and guide action and behavioral change.

\section{Incentivization to reward behavior}


Incentivizing desirable behavior is another route to bring about behavioral change (e.g., Bijleveld \& Aarts, 2014). The idea is simple: if the individual displays the desirable behavior, he will be rewarded with either a financial benefit or other material or non-material means that have a motivational value to him. The reward functions as a motivational factor, reinforcing the individual's intention to engage in the desired behavior as a means to obtain the reward. The caveat that comes with this method is that it is entirely based on extrinsic motivation and its effects are thus short-lived. As the incentive is taken away, the motivation and intention to display the behavior is removed as well (Gneezy et al., 2011).

A more sustainable means to attain lasting behavioral change is through targeting intrinsic motivation (Rigby et al., 1992). If the individual is motivated intrinsically, the motivation to change his behavior occurs irrespective of an external reward and is thus less affected by changes to the reward structure (Ryan \& Deci, 1985). To the contrary, if a behavior is intrinsically motivated, the behavior is more likely to persist after the incentive is decreased in value or removed altogether, with intrinsic motivation possibly even being compromised by an incentive (Deci et al., 1999).

Previous research has mainly addressed three basic requirements in order to increase intrinsic motivation: autonomy, competence and relatedness. Higher levels of autonomy, competence and social relatedness are considered to make individuals more confident that they can perform an activity on their own in the social context at hand. In doing so, these studies follow an elaborate procedure and target explicit beliefs and the judgment of agency to safeguard intrinsic motivation of the agent (Cameron \& Pierce, 1994). Whereas this strategy to behavioral change may work well, another perhaps less intensive possibility to target intrinsic motivation by incentivization has been explored in Chapter 5. There, we linked the incentive (in the form of a monetary reward) to the intention to execute an action and showed that such motivation treatment in strengthening an individual's intention positively affects the 
sense of agency. However, whether or not this change by motivating sensorimotor processes of intentional action represents a firm basis for intrinsic motivation, and hence, also has lasting effects on behavior, remains to be tested and warrants further research.

\section{Environmental adaptation}

A third route to achieve behavioral change is through changing the environment. Such adaptations of the environment can include small alterations but often also mean changes on a larger scale. Well-known examples of relatively small environmental adaptations include placing bins to encourage people to keep the park clean or staircases that make sounds when walked upon to encourage people to take the stairs instead of the escalator (Halpern, 2015). More considerable changes include changing the interior of buildings and a city's lay-out and infrastructure. However, whether small or large, it may not always be feasible to implement such environmental alterations as they can come with great adjustments to the environment and can be quite expensive. A major problem though is that they do not change the intentions underlying the behavior (Marteau et al., 2011). For that reason, environmental alterations to change behavior are argued to have added value when the to-be-changed behavior is nonintentional, i.e. habitual (e.g., Aarts et al., 1997; Wood \& Rünger, 2016). Otherwise, other measures may be more effective at achieving behavioral change and less expensive to implement.

It is however difficult to determine when behavior occurs without intentions. In theory, an action that is instigated by cues in the absence of a goal is conceptualized as habitual behavior. Nevertheless, a recent analysis of the habit literature suggests that the evidence for the goal-independency of habits is less solid than has been assumed so far. Most research seems to be designed to test automatic responses to the environment, but the direct role of intentions has not been ruled out properly (Marien et al., 2019). As our studies on 
personal autonomy suggest (Chapter 4), even when the environment forcefully triggers actions, the sensorimotor processes seem to remain intact in shaping intentional binding and the sense of agency. Hence, any attempt to propose environment changes to reform human habits needs to be validated in terms of whether the behavior is driven by genuine habits or not, that is, a breakdown of the minimal self. The present research might offer future research possible ways to explore this issue by examining whether and when cue-driven behaviors are no longer accompanied by action-effect binding that one usually observes for intentional actions. The empirical study of intentional action and the sense of agency is thus not only important for theory building but can also be of benefit when deciding which strategies for behavioral change are most desirable and acceptable to change behavior for the benefit of society.

\section{A final note: The case of coercion}

A general assumption of most behavioral change models is that education, incentivization, and environment adaptation should work in tandem, such that changes in knowledge, motivation, and context have the highest impact on behavioral change. It is important to note, though, that these three pathways still leave open the possibility for the individual to have freedom to act otherwise, a position that concurs with a libertarianpaternalistic outlook on public policy (Sunstein \& Thaler, 2003). A last resort to change behavior, then, is to address measures of obedience. In such cases, the government might implement measures and bans that are embedded in regulatory decisions and institutional law to enforce people to adapt their behavior. The recent outbreak of the COVID-19 disease caused by a novel coronavirus that has turned out to be an unprecedented challenge for governments and public health organizations around the globe, illustrates the importance of obedience and coercion in order to curb a pandemic. 
These radical measures can cause reactance and represent an extreme form of social pressure that can have a powerful negative impact on people's sense of agency. Caspar et al. (2016; 2017), for example, demonstrated that the sense of agency decreases on a sensorimotor level when the agent is directly coerced into harming another person. Milgram (1974) coined this dramatic obedience effect as agentic shift, - the tendency to relinquish personal control to an external agent. That is, the agent distances herself from his behavior, weakening the feeling of agency.

While coercion has a direct impact on the sense of agency, a defining characteristic of humans, however, is their resilience and the ability to adapt to changes in their environment. On a larger scope, the adaptiveness of humans can be observed when their livingcircumstances change. An example is the division of Germany after WWII. At the end of the war the country was divided amongst the victorious forces, which later resulted in the formation of West Germany and the German Democratic Republic (GDR). Despite the name, citizens of the GDR were restricted in many regards and were largely prohibited, for example, from traveling or freely choosing their vocation. Moreover, freedom of speech and the right to assemble were curtailed. Whereas some individuals revolted against these restrictions, many citizens were loyal to the state, supporting it and later defended the restrictions, often stating that they intentionally followed the rules (Pollack, 2004). Soldiers at the border-wall even defended shooting at defectors who tried to escape the GDR, in many cases stating that they wanted to protect their country (Conradt, 2011).

The example of the GDR may be quite extreme, but it illustrates a general capacity of humans to adapt to even drastic restrictions and accept coercion. How do such changes affect a person's sense of agency? It is likely that while the sense of agency is initially diminished by coercion and autonomy restrictions, it might be restored when the agent becomes accustomed to the restrictions (i.e., in an effort to adapt to the lasting coercion). Adaptation 
thus can cause people to experience coercion as rather normal. This might also explain why people experience their actions to be intentional in a society where our daily lives are constrained by rules and laws. Given the essence of coercion as a strong form of social pressure, and the importance of resilience to deal with social challenges, it is intriguing and interesting to further explore how the sense of agency and the feeling of being an intentional agent can be altered when people are forced to change their behavior. The research discussed in the present chapter might offer a firm basis to engage in this scientific endeavor.

\section{Outline of the Dissertation}

The remaining part of the dissertation consists of four empirical chapters. The first two chapters tested temporal perception in the absence of motor action, while the last two chapters closely tested the influence of both undermining and amplifying intentionality. All chapters utilized the intentional binding effect as a marker for the implicit sense of agency. To assess intentional binding, all studies reported in this dissertation used the Libet clock task. The choice of the Libet clock task over, for example, interval estimation tasks, was primarily motivated by its ability to assess the separate shifts in action and effect, allowing for a more accurate examination of temporal perception and the classification of it as temporal binding/repulsion. The remaining empirical chapters of this dissertation are outlined below.

Chapter 2 presents two studies that test whether auditory sensory input is sufficient for temporal binding to emerge when motor action is absent. Additionally, causal belief was manipulated between participants. Instead of temporal binding, a robust temporal repulsion effect was found. Two successive auditory stimuli were shifted away from each other in temporal perception, causing the time interval between them to be perceived as larger. 
Chapter 3 expands on the findings of Chapter 2 and tests the importance of the distinct sensory tactile action-input in temporal binding. A first study tested a novel tactile-based Libet clock task. Again, a robust temporal repulsion effect between a tactile and an auditory stimulus was found when motor action was absent. Two additional studies replicated this repulsion affect and showed that it can be attenuated when the input is predictable in time and identity.

Chapter 4 focused on the relationship between autonomy and the sense of agency. In three studies, the effect of partial, full and no autonomy in action selection and timing on intentional binding as an index for the sense of agency was assessed. Contrary to past research, we did not find any differences in binding magnitude between the three autonomy conditions. Interestingly though, the psychological experience of autonomy was considerably affected by the manipulations. These findings thus suggest that implicit markers of agency are not affected by autonomy restrictions.

Chapter 5 assessed the influence of the intentional strength with which an action is executed. Participants learned to press different keys with varying reward values, with higher reward values hypothesized to strengthen the action intention with which they executed the key press. Earning the reward was entirely dependent on the action, and postdictive modulation of binding was not possible. Results showed a stronger intentional binding effect when participants held a stronger intention as compared to a weaker action intention.

As a final remark: Please note that all the empirical chapters of this dissertation have been written as individual articles for publication in scientific journals. Hence, they can be read separately or subsequently. Overlap in content may occur across chapters. 


\section{Chapter 2}

Intentionality and Temporal Binding: Do Causality Beliefs Increase the Perceived Temporal Attraction between Events?

Based on: Antusch, S., Custers, R., Marien, H., \& Aarts, H. (2020).

Intentionality and temporal binding: Do causality beliefs increase the perceived temporal attraction between events?. Consciousness and Cognition, 77, 102835. 


\begin{abstract}
Intentional motor actions and their effects are bound together in temporal perception, resulting in the so-called intentional binding effect. In the current study, we address an alternative explanatory mechanism for the emergence of temporal binding by excluding the role of motor action. Employing a sensory-based Libet clock paradigm, we examined temporal perception of two different auditory stimuli, and tested the influence of beliefs about the causal relationship between the two auditory stimuli, thus simulating a crucial feature of intentional action. In two experiments, we found a robust temporal repulsion effect, indicating that instead of being attracted to each other, the auditory stimuli were shifted away from each other in temporal perception. Interestingly, repulsion was attenuated by causal beliefs, but this effect was fragile. Furthermore, temporal repulsion was unaffected by the intensity of prior learning. Findings are discussed in the context of intentional action awareness research and multisensory integration.
\end{abstract}


The ability to integrate sensory information into a coherent picture of the world is an important human trait. It enables people to build a conscious representation of complex visual scenes and auditory events that are otherwise disconnected in time or space (Serences $\&$ Yantis, 2006; Shamma et al., 2011). Apart from integrating perceptual input, humans also form coherent representations of their own behavior (Aarts \& Custers, 2009; Vallacher \& Wegner, 1987). Self-produced actions that predict and result in sensory feedback, such as pressing keys that result in a sound or light, are perceived as coherent events of meaningful behavior (e.g., playing a tone on the piano or illuminating a room), even though action and effect are spatially and temporally separated and do not form a single entity.

This ability to construct a coherent conscious experience of one's own behavior has important implications for the understanding of the self and the sense of agency, i.e. the feeling of causing one's own action and effects in the external world. Specifically, people perceive their intentional actions and resulting outcomes as if they are temporally bound together in conscious awareness; a phenomenon that is referred to as intentional binding (Haggard et al., 2002b). It is assumed that this temporal binding effect is rooted in internal prediction processes resulting from intentionally selected motor actions, causing the compression of the time interval between action and effect and the formation of a coherent representation of the action-effect event (Frith et al., 2000).

Recent research, however, suggests that this temporal binding effect might not be the result of intentionality per se (e.g., Desantis et al., 2012; Dogge et al., 2012; Hughes et al., 2013), but rather a product of other factors that are associated with intentional action. In the present study, we examine this subject in more detail and raise the question whether the temporal binding effect is similarly sensitive to events that do not require any form of action. Building on the notion that intentional actions are associated with beliefs in causality that might bias the perceptual relation between two events (Buehner \& Humphreys, 2010; 
Desantis et al., 2011; Eagleman \& Holcombe, 2002), we tested whether causality beliefs cause two successive auditory stimuli to be perceptually bound together in the Libet clock paradigm. The emergence of such a binding effect as a function of causality beliefs would undermine the validity of the temporal binding task as a read-out of intentionality and a measure of sense of agency.

One central argument that has been put forward in favor of the central role of intentionality in action concerns the impact of externally induced action on temporal binding. Some studies employed transcranial magnetic stimulation (TMS) to induce an involuntary action followed by a tone (Haggard et al., 2002b; Haggard \& Clark, 2003). Subjects judged the time of movement onset and tone onset by using the Libet clock. The time judgments did not reveal a temporal binding effect but a clear and strong repulsion effect instead, indicating that subjects perceived the motor movement and subsequent tone as two separate events.

While these TMS experiments suggest that the temporal binding task can distinguish involuntary from voluntary action in producing a coherent representation of one's own action, it is important to note that the magnetic stimulation of motor areas results in an abrupt, reflexive and oddly felt movement of a limb (here a finger). Clearly, human agents have the capacity to evoke actions by direct brain stimulation, but such action onset does not fit with everyday externally induced motor movement where intentionality is ruled out, for example when someone else pushes or pulls one's hand into a specific direction. Building on this notion, recent studies show that temporal binding might be reduced but does not vanish for actions that are not self-chosen or driven by internal motivation (Antusch et al., 2019; Caspar et al., 2016) or passively induced in the agent by another experimenter or a mechanical lever press (Dogge et al., 2012; Borhani et al., 2017; Engbert \& Wohlschläger, 2007; Moore et al., 2009). These findings suggest that it is not intentionality per se that drives the temporal 
binding effect, as externally induced movement does not cancel out temporal binding in settings that include clear causal relations between action and outcome.

One explanation for the observed binding between externally induced actions and a tone might be that participants were able to predict the outcome and believed to have a causal role in producing it. Causal beliefs impact cognitive processes at several stages, e.g., learning, judgment and decision-making (Lucas \& Griffiths, 2010; Harris et al., 2020). Moreover, higher-order causal beliefs have been shown to affect low-level processes of perception (Buehner \& Humphreys, 2009; Buehner \& Humphreys, 2010; Cravo et al., 2009). A study by Buehner and Humphreys (2009), for example, suggests that mentally linking one's key press to a subsequent tone following a presumed causal association enhances the anticipation of the tone. However, not only effects following from motor actions are subject to temporal binding, also external (visual) events can be spatially bound together when they share a common causal association (Buehner \& Humphreys, 2010). Accordingly, holding a higher-order belief about the causal relationship between two events increases binding between them, even when action is not actively involved.

In a further exploration of the role of causality beliefs in temporal binding of involuntary movement and effect, Dogge et al. (2012) subjected their participants to a temporal binding task with the Libet clock. Participants either executed a voluntary key press or an involuntary key press that was induced by a lever pulling down their index finger. In the involuntary conditions, some participants were asked to consider themselves as the agent, construing the resulting effect (tone) as being caused by their key press. The magnitude of the binding effect increased between the simple involuntary and involuntary self-causation condition, with mean judgment errors (indicating a stronger binding effect) being larger in the involuntary self-causation condition (Dogge et al., 2012). Similarly, it has been shown that explicit beliefs of being the causal agent resulted in stronger intentional binding in 
participants in a joint action task (Desantis et al., 2011). Whereas informative, these studies do not entirely rule out the role of motor prediction and intentionality in temporal binding between action and effect. It is for example conceivable that participants started up their motor system once they noticed a slight movement of the mechanical lever, causing them to experience a motor prediction signal and/or intentionality over the key press. Because of the tight link between perception and action (e.g., James, 1890; Prinz, 1997; Decety \& Grezes, 2006), a similar reasoning might account for findings that show binding effects as a result of observing others' action (e.g., Desantis et al., 2012; Kirsch et al., 2019).

A more stringent test of the role of causality beliefs in temporal binding is provided by sensory paradigms that do not employ motor action. In one such study by Haggard and colleagues (2002a), participants judged the temporal onset of two identical successive auditory stimuli that were separated by a $250 \mathrm{~ms}$ time interval. Results revealed that especially the second stimulus was temporally repulsed from the first stimulus (Haggard et al., 2002a). Similar results were obtained by Desantis et al. (2012) who compared the temporal perception of two non-motor stimuli separated by a $400 \mathrm{~ms}$ time interval against an operant action condition in a Libet clock paradigm. The findings were such that both, congruent and incongruent (with precedingly learned associations), tone pairs resulted in temporal repulsion instead of temporal binding, suggesting that temporal contiguity and identity prediction were not sufficient for binding to emerge (Desantis et al., 2012). The first tone was perceived as occurring earlier than in actuality, and the second stimuli was perceived as occurring later than in actuality, leading to a repulsion instead of a binding effect. Thus, instead of integrating the two stimuli such as in intentional binding, participants seemed to detach the two from each other by experiencing them as two separate entities and shifting them away from each other in temporal perception. 
It is important to note that these studies did not investigate the role of causal beliefs and might have suffered from flaws that reduced the probability of observing a temporal binding effect. For example, Haggard et al. (2002a) used successive tones of the same frequency and length. Hence, there might have been little inclination for participants to integrate the tones into a coherent event and to perceive them as causally related. Thus, participants perceptually shifted the tones away from each other. Using tones of different frequencies, Desantis et al. (2012) paired the tones in congruent and incongruent withinsubject blocks and employed an unconventional $400 \mathrm{~ms}$ time interval. Because the tones were sometimes congruent and sometimes incongruent, a stable predictive link could not be formed. Furthermore, it is unclear how the extended time interval affected the results. Finally, while suggesting temporal repulsion, comparisons with the baseline tone condition in the Haggard et al. (2002a) study only suggests temporal repulsion of the second tone, while the first tone seems to even be bound to the subsequent tone. Desantis et al. (2012) only tested the temporal perception of the second tone while a comparison baseline condition was lacking completely. Hence, the interpretation of the findings as demonstrating temporal repulsion was neither statistically tested nor can it be claimed in the absence of a comparison baseline condition. Thus, it remains subject to empirical scrutiny whether external stimuli in successive contexts are repulsed or bound to each other in temporal perception.

The present study therefore aimed to replicate the temporal binding (repulsion) effect with two different auditory stimuli by employing a unimodal sensory paradigm based on the Libet-clock. In our task, participants acquired the associations between two auditory stimuli pairs, such that the first stimulus predicted the onset of the second one. Each pair consisted of a noise sound (such as a scratch sound) followed by a sinusoidal tone $250 \mathrm{~ms}$ later. The pairings remained constant for each participant, allowing for participants to perceive the stimuli as being linked and represented as one event. Furthermore, as an important addition to 
earlier work, we examined the role of causality belief in the perceived temporal attraction between the two auditory stimuli. To that end, we explicitly manipulated beliefs about the causal relationship between the two successively presented auditory stimuli to more stringently test their influence on temporal binding of the stimuli. The first experiment addresses the basic role of perceived causality in modulating the temporal binding between two different auditory stimuli. Experiment 2 was designed to further explore the role of perceived causality in a context where the association between the first and second auditory stimulus was strengthened by practice.

\section{Experiment 1}

\section{Methods}

\section{Participants and Design}

In total, $64^{3}$ individuals (49 females), with a mean age of 23 years $(M=23.02, S D=$ 6.15) participated in the experiment in exchange for course credit or monetary reimbursement. Participants were randomly assigned to one of two between-subject conditions, resulting in 33 participants in the control condition and 31 participants in the causal belief condition. The study was carried out in line with the guidelines of the Declaration of Helsinki and was approved by the Ethics committee of the Faculty of Social and Behavioral Sciences at Utrecht University as part of a project line (ethics approval code: FETC17-124). All participants gave written informed consent.

\footnotetext{
${ }^{3}$ As to the best of our knowledge, no data for a comparable effect in a stimulus-based paradigm was available at the point of data collection. For a-priori sample size calculation in G*Power 3.1 (Faul et al., 2007), we first used the following parameters: $\mathrm{f}=.25, \alpha=.05$, power $=.80$, nonsphericity correction $\varepsilon=1$ and $r=.5$ to determine the sample size in an ANOVA: Repeated measures within-between interaction. This yielded an estimated sample size of 24 participants. Given a moderate effect size and a lower power than the default of .90 in G*Power 3.1, we wanted to avoid power issues for testing effects in the within-between subject design. Thus, we aimed at a sample size of at least $n=60$ (we could run a few more within the time frame of our lab availability).
} 
A 2 (target: noise vs. tone) x 2 (type of trial: baseline vs. succession trials) x 2 (condition, between: control vs. causal belief) mixed design was employed.

\section{Procedure}

The experiment consisted of an altered Libet clock paradigm as used by Haggard et al. (2002) and was programmed using Eprime Software version 2.0. Four different auditory stimuli of $100 \mathrm{~ms}$ length were used: two noise sounds and two sinusoidal tones. The noise sounds resembled scratch sounds while the sinusoidal tones were of either $300 \mathrm{~Hz}$ or $1000 \mathrm{~Hz}$ frequency ${ }^{4}$. All stimuli were played via a Sennheiser headphone.

Acquisition phase. The experiment began with an acquisition phase in which participants were familiarized with the auditory stimuli and their combinations. At the start, participants were told that four different auditory stimuli would be used in the experiment and that they would begin with experiencing each stimulus once. While participants experienced a stimulus, an on-screen message stated which stimulus they were experiencing. By pressing F1, they could advance to hearing the next stimulus. Subsequently, to facilitate the distinguishability of the stimuli, participants experienced each stimulus four additional times. These trials advanced automatically and like in the preceding trials, it was stated on screen which stimulus participants were experiencing.

After participants were familiarized with the different auditory stimuli, they learned that each noise sound would be paired with one of the sinusoidal tones during the experiment. In the control condition, participants were told that the computer would determine the combinations of the noise and tone, and that they would therefore be independent of each

\footnotetext{
${ }^{4}$ Both random noise sounds were generated using the following website: https://www.wavtones.com/functiongenerator.php. An amplitude alteration was applied to make the noises distinguishable from each other. The noise sounds can be found here:

https://www.sciencedirect.com/science/article/pii/S1053810019302843
} 
other. Put differently, the pairs of auditory stimuli would not be causally related. They then heard the independent noise - tone combinations. In the causal belief condition, participants learned that the computer would choose the noise that would cause the second tone to occur. The second tone would thus be dependent on the selection of the noise. Put differently, the pairs of auditory stimuli would be causally related. They then heard the dependent noise tone combinations. The noise - tone combinations were counterbalanced across participants but remained constant for a given participant.

In both conditions, the noise - tone combinations were presented five times each (i.e., ten trials in total). Participants were asked to pay close attention. They then completed eight trials in which they heard noise - tone combinations that were either congruent or incongruent with the previously learned pairs. In the control condition they were asked to indicate which combinations of tones were presented before, whereas in the causal belief condition, they were asked to indicate which combinations of tones were causally related. This was done to further emphasize and boost the independent (control condition) and the dependent nature (causal belief condition) of the stimuli pairs in the respective conditions before the start of the actual experiment.

Experimental trials (altered Libet clock task). Next, participants commenced with the experimental trials. In both conditions, participants completed four blocks in randomized order. The blocks consisted of two baseline blocks (baseline noise, baseline tone) and two succession blocks (succession noise, succession tone). Each block comprised 24 trials, including four practice trials and 20 experimental trials. The practice trials preceding the experimental trials of each block were identical to and indistinguishable from the experimental trials but were not analyzed. 
Each trial began with the presentation of a dotted clock face consisting of forty grey dots arranged in a circle around the center of the screen, with a diameter of six centimeters. A black dot served as a clock hand and was moving alongside the grey dots with a speed of $2560 \mathrm{~ms}$ per rotation. The starting position of the clock hand varied between trials and was random. Participants were asked to attend to the clock face and the rotating clock hand. Depending on the trial, they then either perceived a single auditory stimulus or a stimuli pair in succession. The onset of the stimuli was programmed to always occur during the second rotation of the clock hand.

In baseline blocks, participants either perceived one of the two noise sounds (baseline noise) or either of the two sinusoidal tones (baseline tone). In the succession blocks, participants always perceived an initial noise sound followed by the paired sinusoidal tone at $250 \mathrm{~ms}$. After the presentation of the last stimulus, the clock hand continued moving for a duration of $1000 \mathrm{~ms}$ before it disappeared. Subsequently, the mouse cursor as well as a prompt to make a time judgment appeared in the center of the clock face. Participants were required to judge either the temporal onset of the noise sound (succession noise trials) or the temporal onset of the sinusoidal tone (succession tone trials) by clicking on one of the dots to indicate the position of the clock hand at the time of the event. In baseline trials, participants always judged the onset of the single presented stimulus (noise in baseline noise and sinusoidal tone in baseline tone trials).

While blocks and trials were identical in both between-subject conditions, instructions differed. In the causal belief condition, instructions to the individual blocks emphasized the causal and dependent relationship of the two stimuli on each other. For example, participants in the causal belief condition read that they would hear two stimuli in succession, meaning that the tones were dependent on each other and that the first noise would cause the following 
sinusoidal tone. In the control condition, in contrast, the independence of the stimuli was emphasized.

At the end of the experimental trials, participants filled in their age and gender. Moreover, participants also answered a question concerning their belief in the causal dependence of the second stimulus on the first stimulus (as indicated on a 9-point Likert scale ranging from 1 - absolutely not to 9 - very much). This question served as a manipulation check. Finally, participants were debriefed and thanked for their participation as well as reimbursed.

\section{Data analysis plan}

Prior to the main statistical analyses, extreme temporal judgments were excluded on a trial basis. Following Aarts et al. (2012), temporal judgments, which were more than $+/-640$ ms away from the actual temporal onset, were excluded as it can be assumed that participants either rated the onset of the wrong stimulus or were inattentive in general on those trials. Overall, the number of judgments excluded based on this criterion was very small $(.14 \%$ of all judgments).

Based on earlier research suggesting a temporal repulsion effect between auditory stimuli (Haggard et al., 2002a; Desantis et al., 2012), we first aimed to test whether there was a significant overall temporal repulsion effect independent of condition. First, for each trial a judgment error (in milliseconds) was calculated as the difference between the judged time of an event and its actual time of occurrence. A positive judgment error corresponds to delayed awareness of the event, and a negative judgment error corresponds to anticipatory awareness. Next, for each participant separate shifts for the first (noise) and second (sinusoidal tone) stimulus were computed by subtracting the mean baseline judgment errors from the mean judgment errors in succession trials (noise shift = mean judgment error in succession noise 
trials - mean judgment error in baseline noise trials; tone shift $=$ mean judgment error in succession tone trials - mean judgment error in baseline tone trials). Finally, overall binding scores were computed by subtracting the shift of the second stimulus from the shift of the first stimulus (i.e., overall score $=$ noise shift - tone shift). Positive overall binding scores thus indicated temporal compression of the interval between the stimuli (temporal binding) while negative scores were indicative of temporal repulsion. See Figure 1 for the distribution of the raw data in the full sample.

First, we examined the direction of the overall temporal shift. To that end, we conducted a one-sample t-test of the overall binding score against zero. As we had no hypothesis pertaining to either a temporal binding or a temporal repulsion effect, we conducted a two-tailed test. Next, we aimed to test whether temporal perception of the stimuli in the two conditions was statistically different. Specifically, we assumed that stimuli in the causal belief condition would be bound more strongly than in the control condition. In line with that, we conducted a one-tailed paired samples t-test on the overall binding scores in both conditions. Finally, we tested the direction and significance of the temporal shift in the two conditions. That is, we tested whether the perceptual shift was equally present in both conditions. For that purpose, two one-tailed one-sample t-tests of the overall binding scores in each condition against zero were conducted.

Additionally, to illustrate the strength of the evidence and confirm the Frequentist analyses, equivalent Bayesian analyses were performed. For the convenience of the reader, complete Bayesian analyses are reported. 


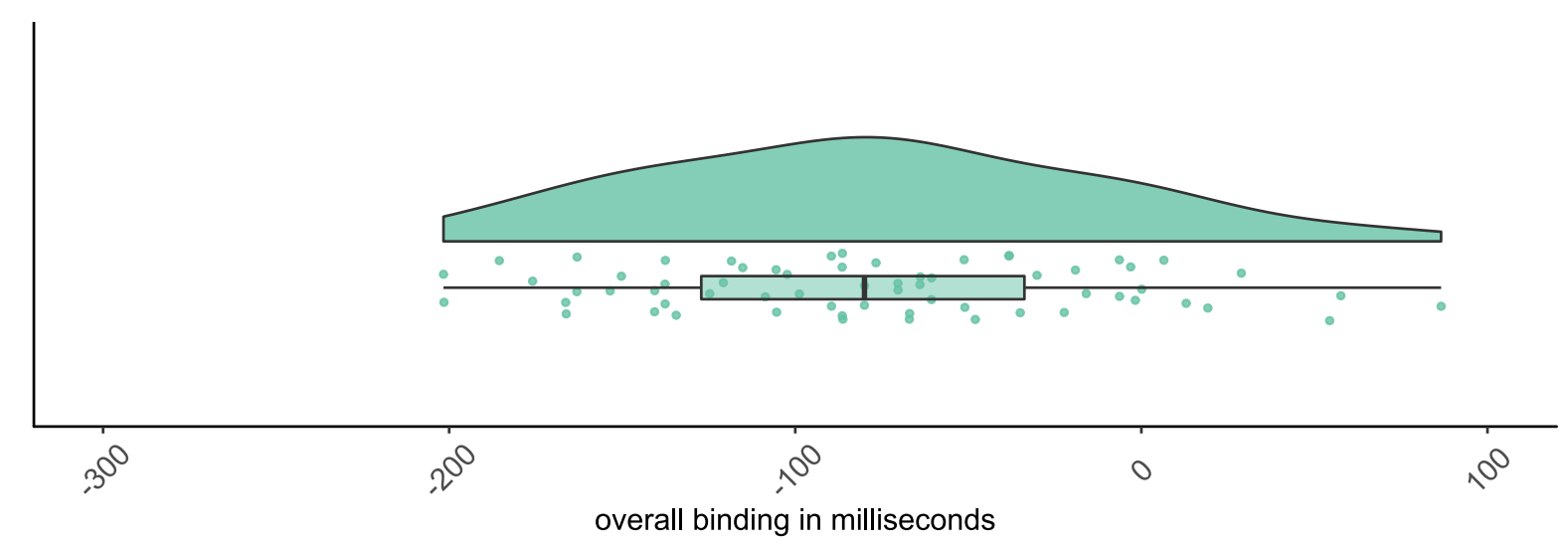

Figure 1. Distribution of the binding scores in ms in Experiment 1 for the whole sample.

Boxplot summaries are based on the median.

\section{Results}

\section{Manipulation check}

Prior to the main analysis, we conducted an independent samples t-test, testing the belief in causality rating between control and causal belief condition. Confirming our manipulations, the results revealed that participants in the causal belief condition $(M=6.68$, $S D=2.15$ ) believed more strongly in the causal dependency of the second stimulus on the first stimulus than participants in the control belief condition $(M=5.24, S D=2.48), t(62)=$ $2.4688, p=.016$, two-tailed, $d=.61,95 \%$ CI $[-1.12,-.11]$.

\section{Main analyses}

Next, we proceeded with the main analysis as outlined in the data analysis plan.

Overall temporal repulsion. To examine the direction of the perceptual shift and its statistical significance, a one-sample t-test of the overall binding scores against zero was conducted. Results revealed a highly significant repulsion effect equaling $77 \mathrm{~ms}(M=-77.20$, $S D=65.94), t(63)=-9.366, p<.001$, two-tailed, $d=-1.17,95 \%$ CI $[-1.49,-.85]$. This temporal repulsion effect occurred bi-directionally. That is, noises were significantly more 
anticipated on succession trials $(M=13.12, S D=46.97)$ as compared to baseline trials $(M=$ 50.97, $S D=47.94), t(63)=5.949, p<001$, two-tailed, $d z=.74,95 \%$ CI $[.36, .80]$. Similarly, tones were perceived significantly later on succession trials $(M=90.17, S D=49.34)$ than on baseline trials $(M=40.83, S D=50.82), t(63)=-6.932, p<.001$, two-tailed, $d z=-.87,95 \% \mathrm{CI}$ $[-1.31,-.66]$

Difference in temporal repulsion between conditions. In addition, to test whether temporal perception differed for participants in the control and the causal belief condition, overall binding scores were subjected to a separate independent-samples t-test based on condition. The results revealed that participants in the causal belief condition $(M=-52.50, S D$ $=67.24, N=31$ ) perceived the stimuli as significantly closer to each other in time than participants in the non-causal control condition $(M=-100.40, S D=56.35, N=33), t(62)=-$ 3.096, $p=.0015$, one-tailed. The difference of $-47.9 \mathrm{~ms}$ was of moderate effect size, $d=-.77$, $95 \%$ CI $[-1.28,-.26]^{5}$. See Figure 2 for a visualization of the overall shifts for the separate between-subject conditions.

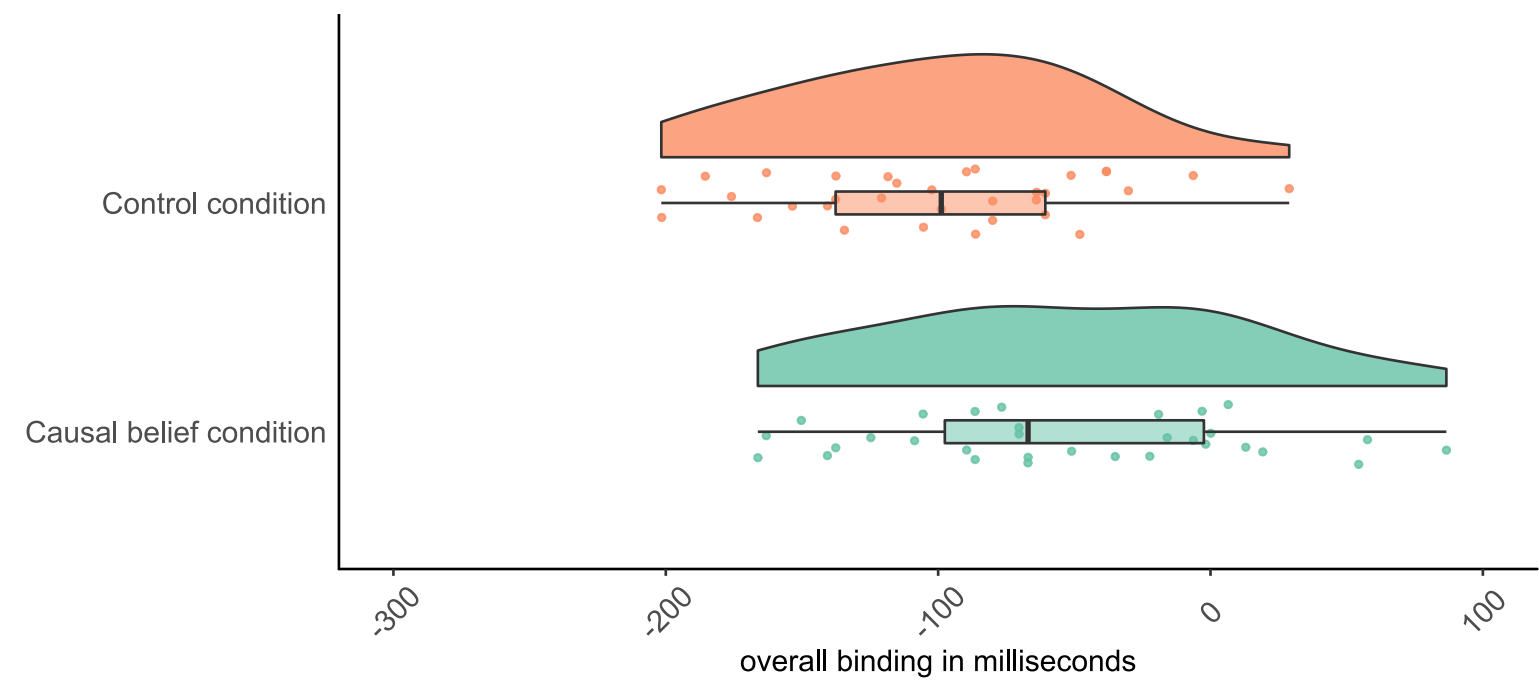

\footnotetext{
${ }^{5}$ Note that this effect equals the three-way within-between interaction effect between target, type of trial and condition in a repeated measures ANOVA, $F(1,62)=9.58, p<.003, \eta_{\mathrm{p}}{ }^{2}=.134, \mathrm{f}=.39$.
} 
Figure 2. Distribution of the binding scores in ms in Experiment 1 in the between-subject conditions. Boxplot summaries are based on the median.

Temporal repulsion per condition. Following, two one-sample t-tests were conducted to test whether the judgments were significantly different from zero. In the control condition $(M=-100.40, S D=56.35)$, judgments showed a statistically significant temporal repulsion effect, $t(32)=-10.24, p<.001$, one-tailed, $d=-1.78,95 \%$ CI [-2.33, -1.22].

Similarly, also in the causal belief condition $(M=-52.50, S D=67.24)$, individuals showed a significant temporal repulsion effect, $t(30)=-4.35, p<.001$, one-tailed, $d=-.78,95 \%$ CI [$1.18,-.37]$. The separate shifts for the noise and the tone in the between-subject conditions are visualized in Figure 3.

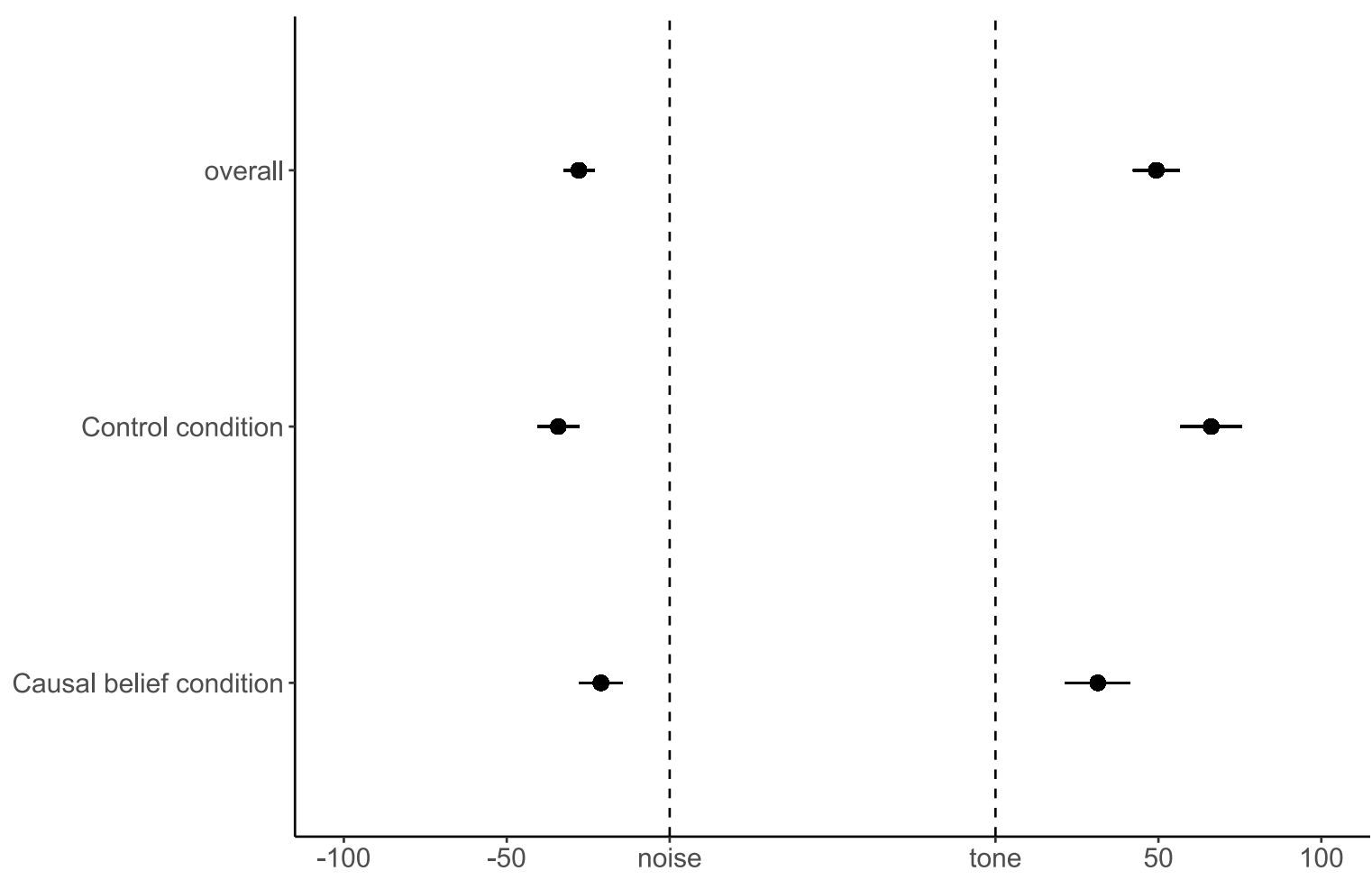

Figure 3. Separate temporal shifts from baseline in ms for Experiment 1. Dots denote the mean, bars represent standard errors.

\section{Bayesian Analyses}


To assess the strength of the evidence, we performed Bayesian analyses in JASP (JASP Team 2018, Version 0.9). In the absence of sufficient information regarding a reasonable effect size and given the novelty of the paradigm, the default Cauchy prior of .707 instead of an informed prior was used for the Bayesian t-tests (Bartlett, 2018; Quintana \& Williams, 2018). To assure the robustness of Bayes Factors, robustness checks were conducted. The results indicated that the chosen prior did not inflate the Bayes Factors. Hence, the results of all tests can be assumed to be robust. The results of all robustness checks are reported in the Supplementary Materials.

Bayesian t-test on overall repulsion. To test the strength of the temporal repulsion effect, we conducted a Bayesian two-tailed one-sample t-test on the overall temporal repulsion score. The alternative hypothesis stated that the temporal repulsion effect differed from zero (equaling no perceptual shift). The results revealed a $\mathrm{BF}_{10}=5.199 \mathrm{e}+10$ 51,990,000,000), 95\% CrI [-1.46, -.84], indicating extreme evidence for the alternative hypothesis.

Bayesian t-test on temporal repulsion between conditions. Next, to test the strength of the difference between the two conditions, a one-tailed Bayesian independent-samples ttest was conducted. The alternative hypothesis assumed that the temporal judgments in the causal belief condition would be smaller than in the control condition, indicating less repulsion. The $\mathrm{BF}_{+0}$ was $25.01,95 \% \mathrm{CrI}[.19,1.19]$, suggesting that the data was 25 times more likely to occur under the alternative than under the null hypothesis. Thus, there was strong evidence for the idea that temporal repulsion was smaller in the causal belief condition than in the control condition.

Bayesian t-tests on temporal repulsion in the separate conditions. Consecutively, two Bayesian one-sample t-tests were conducted. Both overall binding scores were tested 
against a population mean of zero. For the causal belief condition, a $\mathrm{BF}_{10}=183.4,95 \% \mathrm{CrI}[-$ $1.13,-.34]$, was found, indicating very strong evidence for the alternative hypothesis. For the control condition, a $\mathrm{BF}_{10}=7.136 \mathrm{e}+8(=713,600,000), 95 \% \mathrm{CrI}[-2.36,-1.13]$, was found, indicating even extreme evidence for the alternative hypothesis.

\section{Discussion}

In this first experiment, we aimed to replicate the temporal repulsion effect between two auditory stimuli suggested by existing literature (Haggard et al., 2002a; Desantis et al., 2012). Employing a sensory based Libet clock paradigm, strictly excluding the possibility for motor action, for the first time, we empirically tested and statistically demonstrated this temporal repulsion effect (i.e., corroborated by Frequentist and Bayesian analyses).

Additionally, we found that participants who held an explicit belief about the causal dependency of the stimuli on each other, perceived the stimuli as being less strongly repulsed than participants who did not hold such belief. This finding suggests an increased perceived coherence between the stimuli in the causal belief condition. However, temporal binding was still absent, indicating that the temporal integration of the two auditory stimuli was weak and thus did not produce a full coherence experience.

\section{Experiment 2}

To test the reliability of the temporal repulsion effect in the perception of successive auditory stimuli and the attenuation thereof in the causal belief condition, a second experiment was set up. It is conceivable that temporal repulsion persisted in the light of causal belief because of the arbitrary nature of the stimuli and their associations. For example, recent research using a different paradigm suggests that stimuli that have a naturalistic link (such as the picture of a hand and a clapping sound) are more easily bound in temporal perception (Thanopoulos et al., 2018). Research suggests that these associative links between stimuli are 
acquired through learning and repeated exposure (Turk-Browne et al., 2008). Especially in the absence of motor action such learning might be pivotal for forming a stable predictive link. Hence, in an attempt to strengthen these associations, we increased the amount of learning trials from ten to sixty trials in the additional learning conditions.

\section{Methods}

\section{Participants and Design}

The sample of the second experiment consisted of $100^{6}$ (66 females) volunteers with a mean age of 22 years $(M=22.22, S D=2.03)$ who participated in the experiment in exchange for course credit or a monetary reimbursement. The study was carried out in line with the guidelines of the Declaration of Helsinki and was approved by the Ethics committee of the Faculty of Social and Behavioral Sciences at Utrecht University (ethics approval code: FETC17-124). All participants gave written informed consent.

The design of the second experiment resembled the design of the first experiment with the addition of another between-subject factor. The complete design was a 2 (target: first vs. second tone) x 2 (type of trial: baseline vs. succession) x 2 (condition, between: control vs. causal belief) x 2 (additional learning, between: no vs. yes) mixed design. The addition of the learning factor resulted in two additional between-subject conditions. That is, participants could be randomly assigned to either one of the following conditions: control condition or causal belief condition (replication of the conditions of Experiment 1), control learning

\footnotetext{
${ }^{6}$ Like in Experiment 1, we started with the following parameters in G*Power 3.1 (Faul et al., 2007): $\mathrm{f}=.25, \alpha=$ .05 , power $=.80$, nonsphericity correction $\varepsilon=1$ and $r=.5$ to determine the sample size in an ANOVA: Repeated measures within-between interaction, including the moderator test of the learning factor as well. This yielded an estimated sample size of 36 participants. As this was again a rather liberal estimation, we decided to increase the power to detect the moderating influence of the learning factor. Hence, we increased the sample size to $\mathrm{n}=100$.
} 
condition or causal belief learning condition (resulting from the addition of the learning factor to the design).

\section{Procedure}

The general procedure of the experiment resembled that of the first experiment. Also the stimuli used were identical to the stimuli in the first experiment. The experiment was programmed using Eprime 2.0. The procedural differences between the first and the second experiment are outlined below.

Acquisition phase. The experiment began with an acquisition phase. In the control and causal belief condition, the same acquisition phases as in the first experiment were employed (for details see Experiment 1).

In the additional learning condition, after having acquired the stimuli, participants completed extensive learning phases of the stimuli pairs. In total, participants completed 60 learning trials ( 2 x 30 trials, separated by a 30 seconds break). The repeated exposure to the stimuli pairs thus would strengthen the association between the stimuli. Like in the first experiment, in the causal belief condition, the causal relationship between the two stimuli of a pair was emphasized whereas in the control condition their independence was emphasized.

Experimental trials (altered Libet clock trials). The control and causal belief condition were identical to the first experiment. The instructions for experimental blocks in the learning conditions were borrowed from the respective control conditions.

\section{Data analysis plan}

As in Experiment 1, we excluded temporal judgments that were more than $+/-640 \mathrm{~ms}$ away from the actual temporal onset of the stimulus on a trial basis (Aarts et al., 2012). Overall, the amount of excluded trials was very small (.15\% of the total amount of 
judgments). Furthermore, we calculated separate shift scores as well as overall binding scores per participant using the same formula as in Experiment 1. Upon inspection of the overall binding scores, one participant seemed to be an extreme outlier with an average mean judgment score of $-532.38 \mathrm{~ms}$. As this score was more than six standard deviations away from the average overall binding score of the remaining participants $(M=-67.28, S D=74.34)$, we decided to remove this person from further analysis. A visualization of the raw data including the outlier is provided in Figure 4.

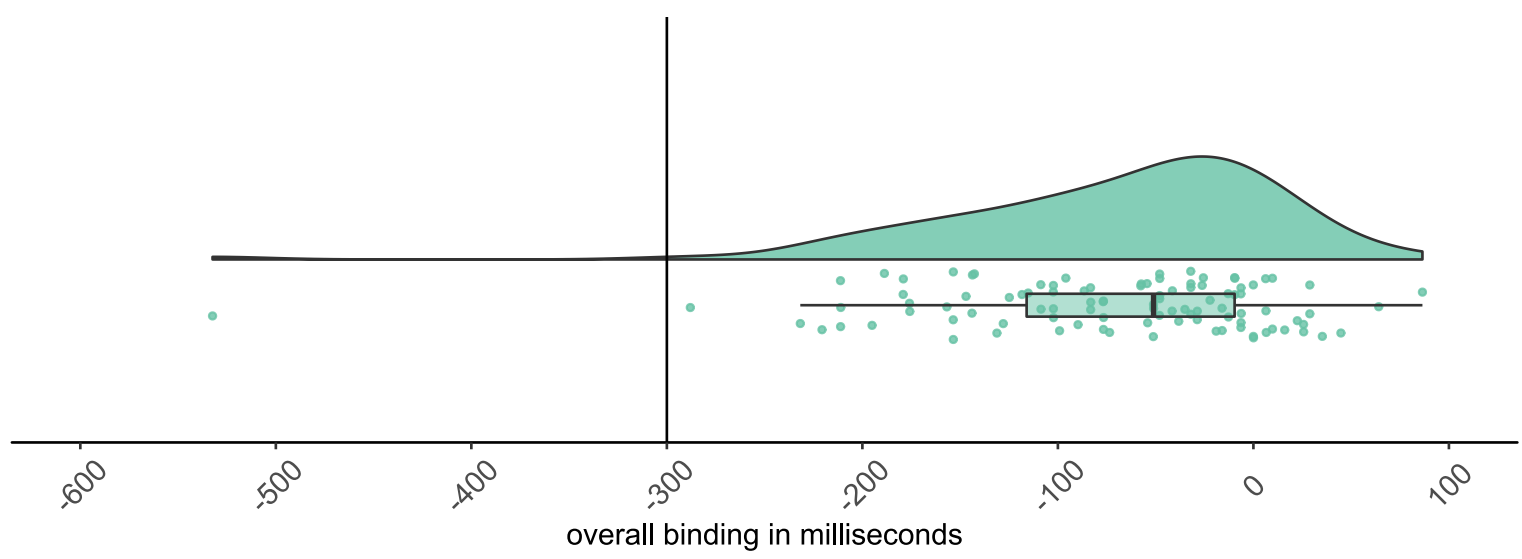

Figure 4. Distribution of the overall binding scores in ms in Experiment 2 for the whole sample. Boxplot summaries are based on the median.

We proceeded with testing the significance of the overall temporal perceptual shift. Since we had no directional hypothesis regarding the perceptual shift in the overall sample (either temporal binding or temporal repulsion), we conducted a two-tailed one-sample t-test on the overall temporal binding scores. After establishing the significance of the temporal repulsion effect in the sample, we subjected the overall binding scores to an ANOVA with causal belief and learning as between-subject factors to assess their influence on temporal perception. Following, four separate one-tailed one-sample t-tests were conducted to test the significance of the temporal repulsion shift in the separate between-subject conditions. 
Finally, equivalent Bayesian analyses are reported to give an impression of the strength of the evidence.

\section{Results and Discussion}

\section{Manipulation check}

To check that the belief in the causal relationship between paired stimuli was indeed stronger in the causal conditions than in the control conditions, we conducted an ANOVA with causal belief condition and learning as between-subject factors on causal belief. The results revealed that, on average, participants in the causal belief conditions $(M=5.6, S D=$ 2.24, assessed on a 9-point Likert scale) believed more strongly in the causal dependency of the second stimulus on the first stimulus than participants in the control belief conditions $(M=$ 3.67, $S D=2.17), F(1,95)=19.615, p<.001, \eta^{2}=.17,95 \%$ CI $[.05, .30]$. Learning was not found to have an influence on the causal dependency belief, $F(1,95)=.101, p=.751, \eta^{2}=$ $.00,95 \%$ CI $[.00, .04]$. Similarly, also the interaction between the causal belief manipulation and learning was not significant, $F(1,95)=1.631, p=.205, \eta^{2}=.01,95 \%$ CI $[.00, .10]$.

\section{Main analyses}

Overall temporal repulsion. A t-test on the overall binding score across conditions was conducted to assess the direction of the perceptual shift and to test its statistical significance. The results revealed a significant repulsion effect of around $67 \mathrm{~ms}(M=-67.28$, $S D=74.34), t(98)=-9.005, p<.001$, two-tailed, $d=-.91,95 \%$ CI [-1.14, -.67]. Simple main effects confirmed that temporal repulsion occurred on both sides - the noise and the tone side, meaning that they were both perceptually repulsed from each other. While noises were perceived as occurring earlier on succession trials $(M=3.49, S D=39.06)$ than on baseline trials $(M=35.44, S D=41.05), t(98)=7.805, p<.001$, two-tailed, $d z=.78,95 \%$ CI $[.31, .73]$, tones were perceived significantly later on succession trials $(M=64.58, S D=60.34)$ than on 
baseline trials $(M=29.25, S D=44.54), t(98)=-6.018, p<.001$, two-tailed, $d z=-.60,95 \% \mathrm{CI}$ $[-.90,-.43]$

Influence of causal belief and learning on temporal repulsion. The overall binding scores were then subjected to an ANOVA with condition and learning as between-subject factors. Neither the main effect for condition, $F(1,95)=.203, p=.653, \eta^{2}=.00,95 \%$ CI $[.00$, $.05]$, nor the main effect for learning, $F(1,95)=.061 p=.806, \eta^{2}=.00,95 \%$ CI $[.00, .03]$, was significant. Moreover, also the interaction between condition and learning was not significant, $F(1,95)=.117, p=.733, \eta^{2}=.00,95 \% \mathrm{CI}[.00, .05]$. Also the planned comparison between the control and the causal belief condition did not yield a significant effect, $F(1,95)=.331, p=.566, \eta^{2}=.00,95 \%$ CI $[.00, .06]$. The causal belief effect that we found in the first experiment was thus not replicated. See Figure 5 for a visualization of the overall binding scores in the four between-subject conditions.

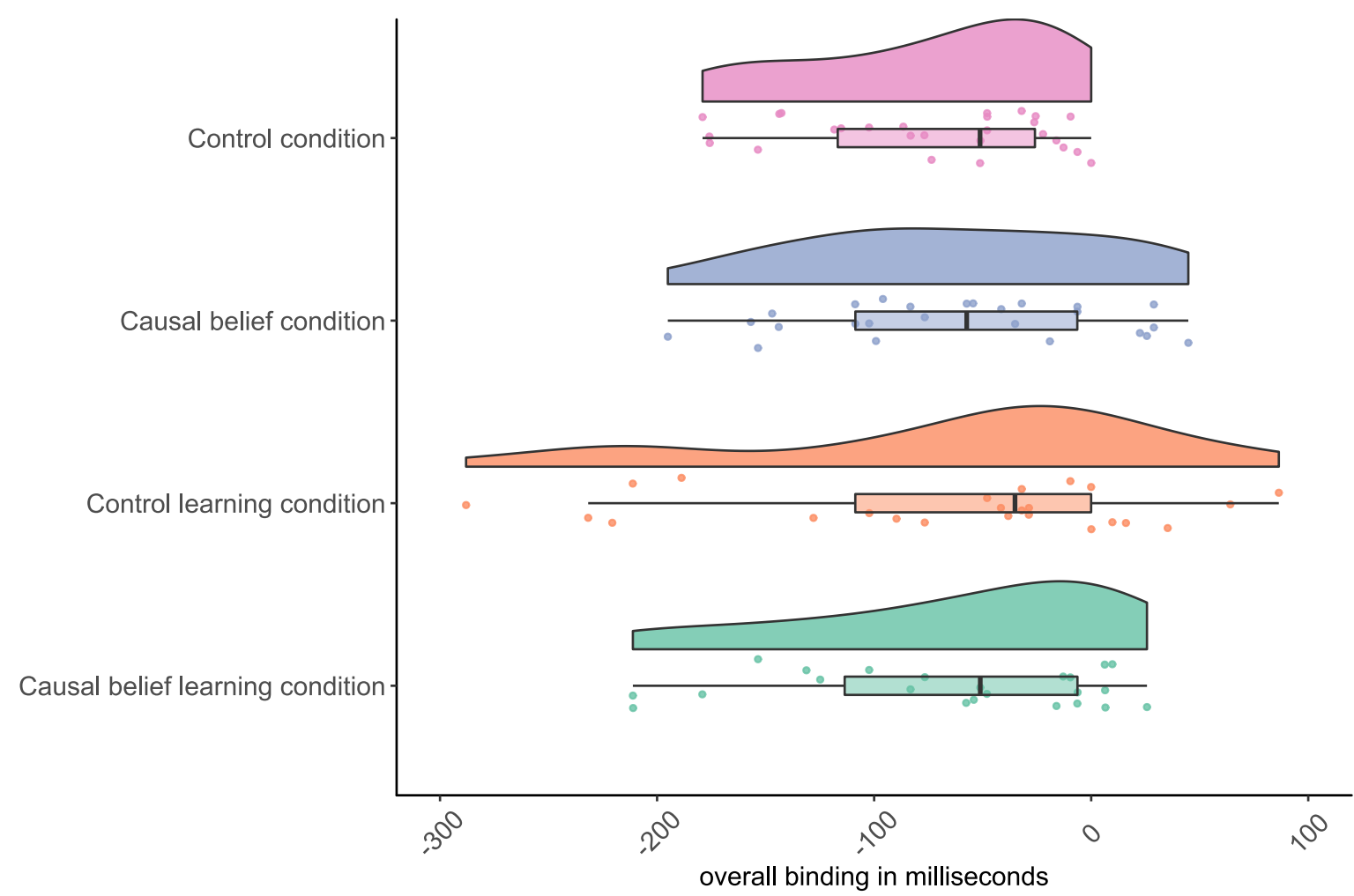

Figure 5. Distribution of the binding scores in ms in the between-subject conditions of Experiment 2. Boxplot summaries are based on the median. 
Temporal repulsion per condition. The scores of all four between-subject conditions were negative, indicating temporal repulsion of the two stimuli from each other: control condition $(M=-74.94, S D=57.46, N=27)$, causal condition $(M=-62.90, S D=68.02, N=$ 25), control learning condition $(M=-66.01, S D=98.73, N=24)$ and causal learning condition $(M=-64.37, S D=73.30, N=23)$. One-sample t-tests were conducted to test if temporal repulsion in the conditions was significantly different from zero. The results were as follows:

$t(26)=-6.777, p<.001$, one-tailed, $d=-1.30,95 \%$ CI $[-1.81,-.78]$ (control), $t(24)=-4.624, p<.001$, one-tailed, $d=-.92,95 \%$ CI $[-1.39,-.45]$ (causal), $t(23)=-3.275, p=.0015$, one-tailed, $d=-.67,95 \%$ CI [-1.11, -.22] (control, learning), $t(22)=-4.211, p<.001$, one-tailed, $d=-.88,95 \%$ CI [-1.35, -.39] (causal, learning). Figure 6 provides an overview of the separate temporal shifts for the perception of the noise and the tone as compared to baseline.

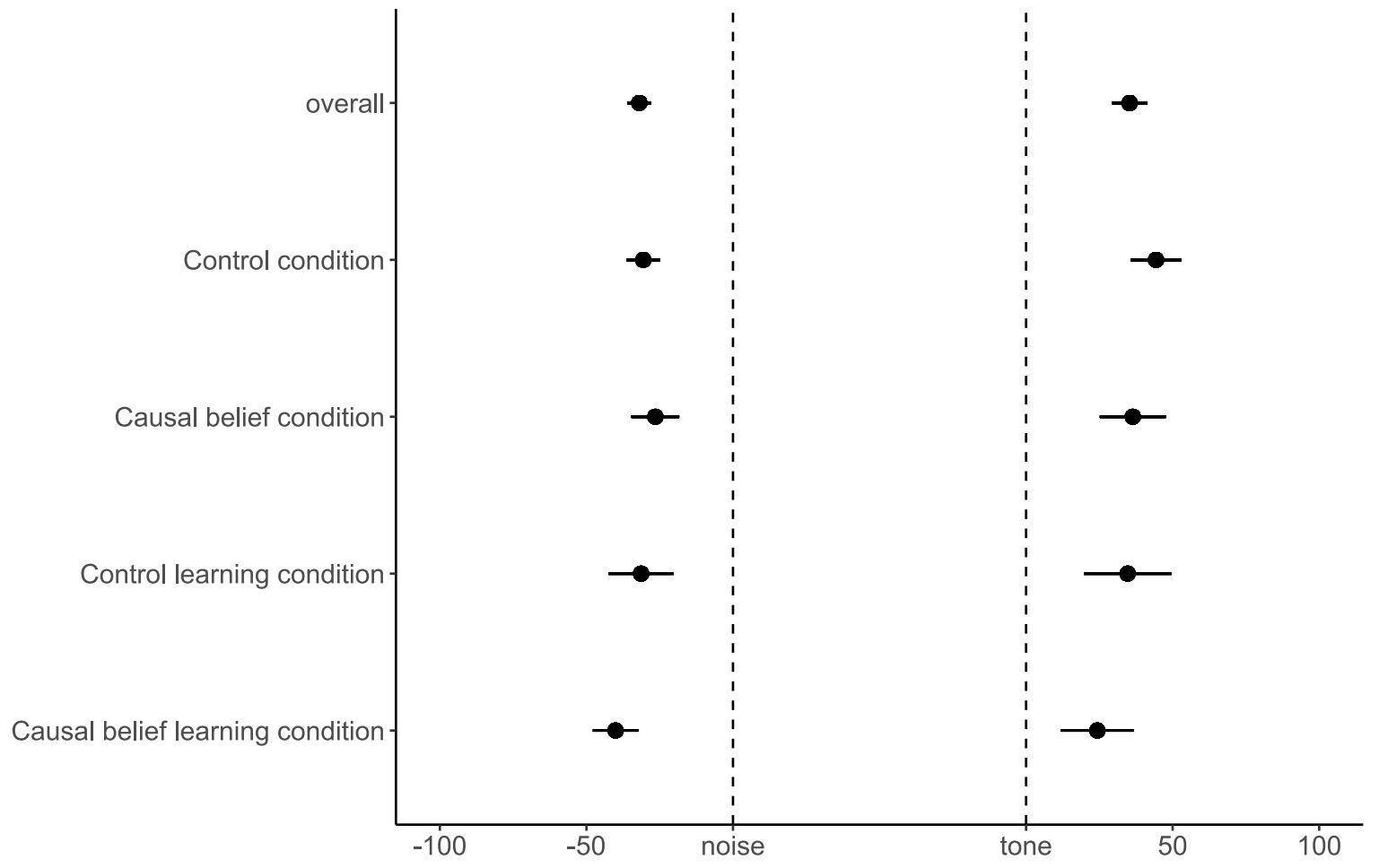


Figure 6. Separate perceptual shifts from baseline in ms for the first and second auditory stimulus in the overall sample and per condition for Experiment 2. Dots denote the mean per condition, bars represent standard errors.

\section{Bayesian analyses}

Furthermore, Bayesian analyses were conducted using JASP software (JASP Team 2018, Version 0.9). Like in Experiment 1, the default Cauchy prior of .707 was used for the Bayesian t-tests as a lack of existing information regarding a realistic effect size did not allow for the determination of an informed prior (Bartlett, 2018; Quintana \& Williams, 2018). To assure the robustness of the Bayes Factors, robustness checks were conducted. The results indicated that the chosen prior did not inflate the Bayes Factors and that all test can be assumed to be robust. The results of all robustness checks are reported in the Supplementary Materials.

Bayesian t-test on overall repulsion score. Like in the first experiment, to test the strength of the temporal repulsion effect, we conducted a Bayesian two-tailed one-sample ttest on the overall temporal repulsion score. The alternative hypothesis stated that the temporal repulsion effect differed from a population mean equal to zero. $\mathrm{The}_{\mathrm{BF}} \mathrm{F}_{10}=$ $3.979 \mathrm{e}+11(=397,900,000,000), 95 \% \mathrm{CrI}[-1.46,-.84]$ indicated extreme evidence for the alternative hypothesis.

Bayesian ANOVA. Subsequently, we conducted a Bayesian ANOVA on the overall binding scores with causal belief and learning as between-subject factors. The results showed that, given the data, there was no evidence that the separate effects of causal belief $\left(\mathrm{BF}_{10}=\right.$ $.234)$ and learning $\left(\mathrm{BF}_{10}=.219\right)$ were a good addition to the null model explaining the extent of temporal binding or repulsion. Similarly, neither a model including both factors $\left(\mathrm{BF}_{10}=\right.$ 
.051) nor a model including both factors and the interaction term $\left(\mathrm{BF}_{10}=.015\right)$ was favorable given the data.

\section{Bayesian t-test on the difference between the control and causal belief condition.}

Next, to test the likelihood of decreased temporal repulsion in the normal causal belief condition as compared to in the normal control condition (replication of Experiment 1) given the data, a one-tailed Bayesian independent-samples t-test was carried out. The null hypothesis that there was no difference between the two conditions was tested against the alternative hypothesis that the temporal repulsion in the causal condition was smaller than in the control condition. The $\mathrm{BF}_{+0}=.497,95 \% \mathrm{CrI}[.01, .7]$, suggested no evidence for the alternative hypothesis.

Bayesian t-tests on temporal repulsion per condition. Finally, we conducted separate Bayesian one-sample t-tests to test each group judgments against zero. The results were as follows: control condition $\mathrm{BF}_{10}=47998.34,95 \% \mathrm{CrI}[-1.75,-.75]$, causal condition $\mathrm{BF}_{10}=250.6,95 \% \mathrm{CrI}[-1.34,-.39]$, control learning condition $\mathrm{BF}_{10}=12.36,95 \% \mathrm{CrI}[-1.05$, -.19], and causal learning condition $\mathrm{BF}_{10}=87.08,95 \% \mathrm{CrI}[-1.23,-.30]$. That is, while there was extreme evidence in favor of the alternative hypothesis in the control and causal condition, the evidence for the alternative hypothesis was (very) strong in the two learning conditions. This suggests that the evidence for temporal repulsion was strong in all conditions but slightly less strong in the learning conditions.

In short, we replicated the temporal repulsion effect of the first experiment, but we could not replicate the moderating influence of causal belief, even when participants received more extensive practice in causally linking the two auditory stimuli.

\section{General Discussion}


Research on action awareness consistently finds voluntary actions and their effects to be attracted to each other in temporal perception. This temporal binding effect is said to be rooted in factors inherent to intentional action - hence the name, intentional binding. Here we explored a potential confound of intentional action in producing temporal binding. Specifically, we explored the role of beliefs of causality, and cancelled out any role of action by examining temporal perception of auditory stimuli. Employing an adapted sensory-based version of the Libet clock paradigm, in two experiments we found a temporal repulsion effect for the perception of auditory stimuli in the absence of motor action. This finding supports past research that observed a similar effect (Haggard et al., 2002a; Desantis et al., 2012). However, we were able to statistically test it with a sufficiently large sample, and unambiguously demonstrated the existence and robustness of such an effect. That is, both the noise and the sinusoidal tone - were significantly shifted away in comparison to baseline.

We also found evidence for a moderating effect of causal belief on temporal binding of auditory stimuli. Specifically, in Experiment 1 we showed that making participants believe that the first auditory stimulus is causally related to the second auditory stimulus reduced the repulsion effect, suggesting an increased form of temporal integration and perceived coherence. The repulsion effect was not fully reduced, and we could not replicate the moderating influence of causal belief in a second experiment, even though we exposed participants to more training in applying causal beliefs to the auditory stimulus combinations. Hence, while we demonstrated a robust and clear temporal repulsion effect for sensory stimuli in the absence of motor action, the influence of higher order beliefs was less robust and not sufficient to cause actual temporal binding, as would be the case in intentional action.

One possible explanation for the absence of a causal belief effect on temporal binding of auditory stimuli might lie in the naturalistic character of the stimuli combinations. Recent research on voluntary action, for example, suggests that naturalistic as compared to non- 
naturalistic links between action-events carry an implicit (learned) causality experience which makes them likely to bind to an effect (Thanopoulos et al., 2018; Dogge et al., 2012; Dogge et al., 2019b). Following this line of reasoning, the absence of temporal binding may be due to the lack of a natural association between the two auditory stimuli. Although previous research showed that spatial binding of visual stimuli could emerge rather rapidly (Buehner \& Humphreys, 2010), auditory sequences might require practice for temporal binding and perception of coherence to occur (Turk-Browne et al., 2008; Hsu et al., 2015; Lange, 2009). We found that the number of trials that we used was not enough and that it is unclear how much prior experience is necessary to induce temporal binding between non-naturalistic stimuli links. While there is research suggesting that a few trials might suffice (Shimi \& Logie, 2018), other research suggests that more elaborate prior learning is necessary (Ernst, 2007). The failure to replicate the causal belief effect on temporal binding thus indicates the fragility of building a coherent representation of a non-naturalistic combination of sounds (Grotheer \& Kovacs, 2014; Jacobsen \& Schröger, 2001), which seems less problematic for intentional action where one produces sounds by key-presses that might be more naturalistic and more easily to acquire.

All in all, the present finding might give reason to suggest that intentionality has a special status in binding action and effect. As far as the evidence in the current literature can tell, temporal binding mainly occurs when people perform, observe and simulate actions (such as key-presses) to produce effects. Despite potential flaws in experimental designs and testing (Hughes et al., 2013), these overall findings strongly suggest that sensorimotor processes are vital to conscious experiences of action coherence and agency. It is unclear, though, whether the predictions involved in this sensorimotor mechanism stem from intentional action, actual body movement or action simulation, or whether temporal binding arises from multisensory causal integration that links physical sensations to predicted sensory effects (Ehrsson et al., 
2004; Prikken et al., 2019). Future research might therefore design temporal binding studies that more indirectly operate on the motor-sensory system, possibly ruling out action simulation and using conditions that resemble sensory experiences (e.g., tactile stimulation of the finger) that often accompany intentional motor action during interaction with the world.

Finally, the present experiments employed the Libet clock paradigm to examine how two successive auditory stimuli are temporally bound together in conscious awareness. A key feature of the Libet clock in a full temporal binding within-subject design is that one can specifically examine whether repulsion (or attraction) is caused by a shift away from the first stimulus or from the second stimuli, or both. Our data suggest that the temporal repulsion effect occurred bi-directionally, such that subjects perceived the first stimulus earlier and the second stimulus later in time than actually was the case. Repulsion in the temporal binding task thus represents an instance in which two successive stimuli are disconnected and separated in conscious experience.

It is important to note that recent research uses alternatives for the Libet clock, in particular time interval estimations. Whereas these time estimations seem to yield findings that correspond with a temporal binding effect, the measure does not offer a clear inspection of the effect: time interval estimations are compared with the actual time intervals, leading to under- or overestimations of time intervals. Notably, in some studies underestimations are considered to represent temporal binding, whereas in other studies overestimations are treated as temporal binding when comparing conditions that are hypothesized to modulate binding effects (Damen et al., 2015; Kuehn et al., 2013; Wen et al., 2015). Thus, although the time interval estimation measure is practical and easy to administer, it is ambiguous in showing whether attraction or repulsion effects are at stake (for studies on time interval estimations with respect to auditory and visual stimuli links, see e.g., Humphreys \& Buehner, 2009; Imaizumi \& Tanno, 2019). Therefore, whereas the Libet clock paradigm has downsides on its 
own (Pockett \& Miller, 2007), it allows for reliable assessments of the relative temporal shifts compared to baseline time perception, which may be preferred over time interval estimation to examine whether, how and when a positive temporal binding (attraction) binding or negative temporal binding (repulsion) effect will occur.

To conclude, the present research clearly shows that two auditory stimuli occurring in the absence of motor actions are not subject to temporal binding but temporal repulsion, even when people believe that the stimuli are causally related. Understanding the role of causal beliefs in binding action and effect has received some attention lately, but most studies still involve motor movement, and hence, it remains unclear whether and how intentional action shapes coherent representations and a sense of agency of our own behavior. We believe that ruling out any role of motor movement (albeit overtly or covertly) might be crucial in examining alternative mechanisms for temporal binding between action and effect, such as multisensory causal binding that integrates associated information from different sensory modalities. The current research might serve as a first step in this enterprise. 


\section{Chapter 3}

Sense of Agency in the Absence of Motor Movement: An Investigation into Temporal Binding of Tactile Sensations and Auditory Effects

Based on: Antusch, S., Custers, R., Marien, H., \& Aarts, H. (under review). Sense of Agency in the Absence of Motor Movement: An Investigation into Temporal Binding of Tactile Sensations and Auditory Effects. 


\begin{abstract}
People form coherent representations of goal-directed actions. Such agency experiences of intentional action are reflected by a shift in temporal perception: self-generated motor movements and subsequent sensory effects are perceived to occur closer together in time - a phenomenon termed intentional binding. Building on recent research suggesting that temporal binding occurs without intentionally performing actions, we further examined whether such perceptual compression occurs when motor action is fully absent. In three experiments, we used a novel sensory-based adaptation of the Libet clock paradigm to assess how a brief tactile sensation on the index finger and a resulting auditory stimulus perceptually bind together in time. Findings revealed robust temporal repulsion (instead of binding) between tactile sensation and auditory effect. Temporal repulsion was attenuated when participants could anticipate the identity and temporal onset (two crucial components of intentional action) of the tactile sensation. These findings are briefly discussed in the context of differences between intentional movement and anticipated bodily sensations in shaping action coherence and agentic experiences.
\end{abstract}


Individuals continuously engage in intentional behavior to control and shape the external world. They select and time their courses of action based on anticipated outcomes and act accordingly. Apart from preparing and executing actions intentionally, humans also form coherent representations of their own behavior (Aarts \& Custers, 2009; Vallacher \& Wegner, 1987). Planning actions and causing effects, such as pressing keys that result in a sound or light, enables their perception as coherent events of meaningful behavior (e.g., playing a tone on the piano or illumining a room). An important contribution to the study of conscious experiences of action coherence comes from research on temporal binding (Haggard et al., 2002b). Intentional actions and their effects are bound together in temporal perception - a phenomenon coined intentional binding. Intentional binding is believed to be intimately linked to the sense of agency, and thus essential for attributing behavior to the self and arriving at feelings of responsibility (Moore \& Obhi, 2012).

While intentional binding is a widely replicated and robust effect, the exact role of intentions remains uncertain, as does the link with sense of agency. Primary support for the link with agency experiences comes from studies using transcranial magnetic stimulation (TMS), showing that abrupt stimulation of cortical motor areas results in temporal repulsion instead of temporal binding between the induced action and a subsequent effect (Haggard \& Clark, 2002; Haggard et al., 2003). Interestingly, TMS studies are among the only studies showing this strong repulsion effect for involuntary actions. In situations in which a person's action is passively induced by other external means, such as a mechanical lever press, temporal binding, though less strong, still emerges (Desantis et al., 2011; Dogge et al., 2012; Kirsch et al., 2019). Furthermore, it even persists when individuals observe intentional actions in (virtual) others (Suzuki et al., 2019; Vastano et al., 2018). These findings suggest that not intentionality per se, but other processes that are associated with intentional action, such as 
knowledge about causality and predictions that one can make based on this knowledge, drive temporal binding (Buehner \& Humphreys, 2010; Desantis et al., 2011).

It is important to note that in passive movement studies, intentional action is not entirely ruled out (Antusch et al., 2020). Participants can mentally simulate their action, or even start up their motor system once they notice a slight movement induced by a mechanical lever, allowing them to build intentions as to select, time and predict the effect resulting from their key press. Because of the tight link between perception and action (e.g., Decety \& Grèzes, 2006; James, 1890; Prinz, 1997), a similar reasoning might explain temporal binding effects as a result of observing others' action.

In the present research we further explore temporal binding in the absence of action. We took on a novel methodological approach that abolishes the action component completely and allows for the examination of temporal binding between a key sensation of action movement and an effect. That is, building on the original Libet clock intentional binding task (Haggard et al., 2002b), we tested whether and when induced tactile sensation in the fingertips, i.e., the sensory input that commonly accompanies key presses, is temporally bound to auditory effects. If temporal binding also occurs between a tactile (fingertip) sensation and a resulting auditory stimulus, this would suggest that simulating and executing a motor movement is not crucial for its emergence. In return, this would also cast doubt on the role of temporal binding as a reflection of a sense of agency.

Recently, research started to explore whether temporal binding between body sensations can occur in the absence of any motor movement. Using a stringent and radical test and employing a single-modal sensory paradigm that rules out any effect of motor-related activity, Antusch and colleagues (2020) examined whether an auditory stimulus (brief presentation of a noise) and another subsequent auditory stimulus (a sinus tone) are 
perceptually bound together in time. Instead of being experienced as attracted to each other, as is the case in temporal binding, a clear temporal repulsion effect was found. That is, the auditory stimuli were experienced as shifted away from each other (cf. Desantis et al., 2012; Haggard et al., 2002a). Importantly, inducing beliefs about causality as well as prior learning of causality between the stimuli did not transform temporal repulsion into binding.

One possible explanation as to why two causally related auditory events are repulsed from each other in temporal perception might be that the predictive auditory cue is exogenous to the agent, thereby reducing the perceived coherence between the two contingent auditory stimuli. It is conceivable that for temporal binding between causally related sensations to occur, an agent needs to process sensory information that usually accompanies endogenous motor movement and is crucial for the feeling of being an intentional agent. An important type of such information is tactile sensation. For example, a finger press on a key not only causes an auditory effect (e.g., an auditory stimulus) but also changes the tactile sensation in the fingertip stemming from the press itself. This movement-related haptic input of selfselected action is discrete and dissimilar from sensory input of the auditory effect that is caused by the action (Aschersleben \& Prinz, 1995; Mates et al., 1992).

Evidence for the importance of tactile sensation in facilitating temporal binding and agency experiences comes from recent research that examined the effects of tactile sensation as part of motor movement. In this research, participants had to produce an effect (e.g., tone) by "touching" their skin or a mid-air interface, thus receiving tactile input followed by the effect. This tactile or mid-air feedback, when compared to conventional button presses or touchpad input, seems to increase sense of agency and temporal binding between tactile stimulus and effect (Bergstrom-Lehtovirta et al., 2018; Cornelio-Martinez et al., 2018; Coyle et al., 2012). Whereas informative, these studies do not directly examine whether tactile sensation in itself enhances temporal binding and the sense of agency. Specifically, they 
allowed participants to intentionally move their finger, thus confounding intentional action and tactile sensation in the absence of action. Accordingly, it remains an open question whether temporal binding between action and effect requires agents to intentionally simulate and actually execute motor movement, or whether tactile sensation suffices for shaping a sense of agency.

Here, we examined the role of sheer tactile stimulation in the sense of agency by systematically testing whether tactile feedback and a subsequent auditory effect are perceptually bound together in time. To assess temporal binding in a context where motor action is entirely absent, we applied a sensory-based adapted Libet clock paradigm that allowed us to measure the subjective compression of the time-interval between tactile sensation and a subsequent auditory effect. Tactile sensation was delivered to a participant's left or right index finger, followed by a high or low frequency tone. Experiment 1 was designed to test the basic effect of our paradigm. Against the backdrop of earlier research on auditory (single-modality) sensation and movement-related tactile sensation (Antusch et al., 2020; Bergstrom-Lehtovirta et al., 2018), both - temporal repulsion or binding - were conceivable findings. Importantly, intentional action is assumed to originate from people's ability to determine the identity and temporal onset of motor movement, that is which action will be selected at which moment in time (Brass \& Haggard, 2008). Taking these crucial agency properties of intentional action into account, we examined the identity feature by explicitly informing participants which tactile fingertip sensation (i.e. either on the left or the right) would occur (Experiment 2), and tested the temporal onset feature by instructing them to carefully use a countdown procedure to anticipate the temporal onset of the tactile fingertip sensation (Experiment 3). In line with earlier research, this agentic information might facilitate temporal binding.

\section{Methods}




\section{Participants and Design}

Twenty-seven ${ }^{7}$ (21 females) volunteers with a mean age of 22 years $(M=22.3, S D=$ 2.98) took part in the experiment for course credit or a monetary reimbursement. All participants gave written informed consent.

The experiment used a 2 (target: tactile vs. auditory stimulus) x 2 (type of trial: baseline vs. succession trials) within-subjects design. All experiments presented in this article were programmed in Eprime Software version 2.0 and got approval from the faculty's Ethics Review Board (ethics approval code: FETC17-124).

\section{Stimuli and Procedure}

Stimuli. Four different stimuli of 100 ms length each were used: (1) a tactile sensation on the left index finger, (2) a tactile sensation on the right index finger, (3) a low tone of 300 $\mathrm{Hz}$ and (4) a high tone of $1000 \mathrm{~Hz}$ frequency. The sinusoidal tones were played via a Sennheiser headphone. The tactile sensations were small vibrations caused by a $50 \mathrm{~Hz}$ sinusoidal signal (inaudible through the headphones) played via an audio exciter (TEAX14C02-8 Compact Audio Exciter, Tectonic Elements) that was mounted on one $\mathrm{cm}$ thick wooden boards.

Acquisition phase. Participants were seated in front of a computer screen in a cubicle. To assure adherence to the instructions, the experimenter remained in the cubicle for the duration of the experiment. All instructions were provided on screen.

Participants put on the headphone and placed their index fingers on the apparatuses positioned right and left of the keyboard (see Figure 1 for the experimental set-up). To

\footnotetext{
${ }^{7}$ Power calculations for all three experiments can be found in the Supplementary Materials.
} 
acquaint them with the stimuli, participants first experienced all four stimuli separately in 24 randomized trials. Next, they learned that each tactile sensation would result in one of the auditory stimuli. The two tactile sensation - auditory stimulus pairings (i.e., left tactile +300 $\mathrm{Hz} \&$ right tactile $+1000 \mathrm{~Hz}$ and left tactile $+1000 \mathrm{~Hz} \&$ right tactile $+300 \mathrm{~Hz}$ ) were counterbalanced in order and across participants but remained constant per participant. Hence, each participant learned two stimuli pairs which were presented five times each in randomized order (i.e., 10 trials in total). Participants were asked to pay close attention to the pairs.

Afterwards, participants were presented with the two learned and two novel stimuli pairs, formed by crossing the combinations (i.e., tactile sensations were presented with the auditory stimulus of the respective other stimuli pair) and asked to correctly identify the learned pairs and negate the novel pairs.

a

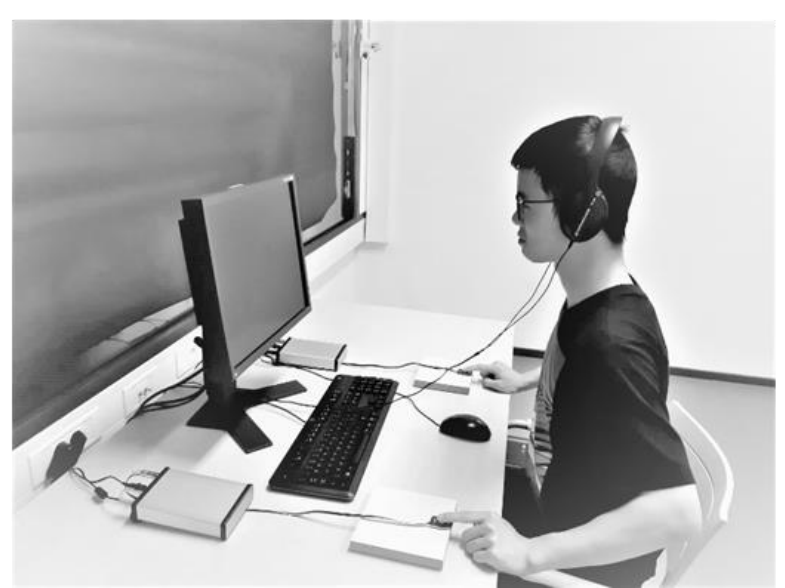

Figure 1. Experimental set-up in the cubicle (1a); apparatus via which the tactile b

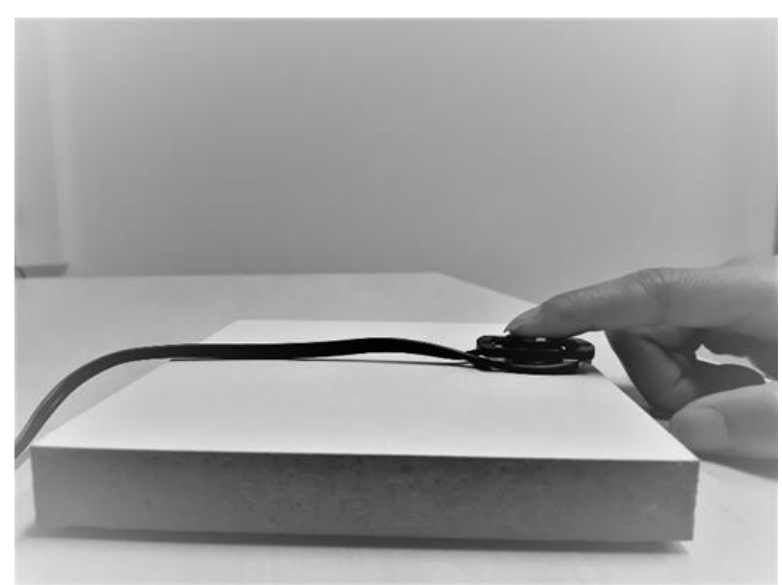

sensation was delivered ( $1 b)$.

Experimental trials (altered Libet clock trials). The experimental sensory-based adapted Libet clock task resembled those commonly used to assess intentional binding (e.g., Haggard et al., 2002a). Participants completed four randomized blocks: a baseline tactile block, a baseline auditory block, a succession tactile block and a succession auditory block. A 
block consisted of 25 trials (five practice trials and 20 experimental trials), resulting in a total of 100 trials. Practice trials were indistinguishable from experimental trials but were not analyzed.

Each trial began with the presentation of a clock face with a rotating clock hand. The clock face had a diameter of six $\mathrm{cm}$ and comprised 40 grey dots arranged in a circle from the center of the screen. A black dot that moved at a period of $2560 \mathrm{~ms}$ served as the clock hand. Per trial, participants received either one stimulus (tactile sensation or tone) or two stimuli in succession (tactile sensation and tone). In baseline blocks, participants experienced either a tactile sensation (baseline tactile blocks) or a tone (baseline tone blocks). In succession blocks, participants experienced a tactile sensation followed by the respective paired tone 250 ms later. The temporal onset of the tactile sensation (or the tone in the baseline tone trials) was programmed to occur at a random moment during the second rotation of the clock hand. After the last stimulus was presented, the clock hand rotated further and disappeared between 1000 and 2000 ms later.

Participants' task was to judge the temporal onset of the tactile stimulation or the auditory (tone) stimulus. Participants estimated the temporal onset of a tactile sensation in baseline tactile and half of tactile -tone trials (i.e., succession tactile trials), whereas they estimated the temporal onset of a tone in baseline tone and half of the tactile -tone trials (i.e., succession tone trials). They judged the perceived onset of the respective stimulus by clicking on one of the dots of the clock face with the mouse cursor. Judgment errors denoting the difference between the actual onset of the stimulus and the estimated onset in milliseconds were logged. The inter-trial interval was $1000 \mathrm{~ms}$. See Figure 2 for a schematic overview of succession trials. 
Finally, participants reported their age and gender, were thanked for their participation, reimbursed and debriefed.

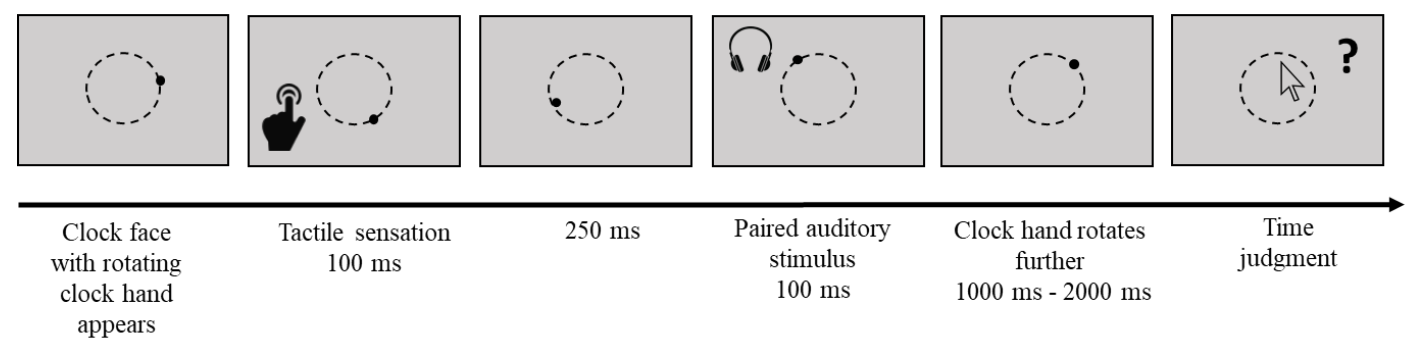

Figure 2. Schematic overview of succession trials in Experiment 1. Baseline trials only included the presentation of a single stimulus (either tactile or auditory).

\section{Data analysis plan}

The data of five participants were excluded because they failed to correctly identify the learned stimuli pairs, resulting in a final sample of 22 participants. Next, for each participant, we calculated mean judgment errors and overall binding scores (see Supplementary Materials for details and formulas).

Next, we continued with the main analysis and subjected the overall binding scores to a one-sample t-test against zero to assess the direction of the effect (i.e., temporal binding or temporal repulsion). Based on the literature either temporal binding or temporal repulsion were possible findings (Antusch et al., 2020; Bergstrom-Lehtovirta et al., 2018) and since we had no clear hypothesis as to the direction of the effect, we decided for a two-tailed test.

Additionally, and to illustrate the strength of the evidence, parallel Bayesian analyses were performed. For the convenience of the reader, complete Bayesian analyses are reported.

\section{Results}


Overall binding. A two-tailed one-sample t-test on the overall shift score $(M=-73.13$, $S D=83.93$ ) against zero was conducted. Results revealed a statistically significant temporal repulsion effect, $t(21)=-4.09, p=.001, d=-.87,95 \%$ CI $[-1.36,-.37]$. Note that this effect equals the two-way within interaction effect between target and type of trial in a repeated measures ANOVA, $F(1,21)=16.70, p=.001, \eta_{\mathrm{p}}^{2}=.44$. Visualizations of the distribution of the overall temporal binding scores and the shifts are provided in Figure 3.

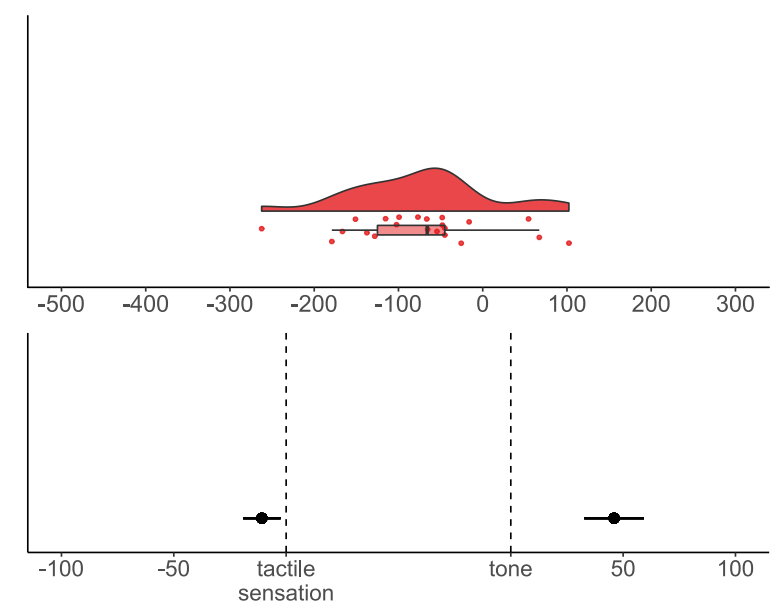

Figure 3. Top: Distribution of the overall temporal binding score (positive scores indicate binding while negative scores indicate temporal repulsion), dots show the distribution of the individual data points and boxplot summaries use the median. Bottom: Separate temporal shifts from baseline, dots denote the mean, bars represent standard errors.

\section{Bayesian analysis}

Bayesian analysis for all experiments were performed in JASP (JASP Version 0.9, JASP Team 2018). As the determination an informed prior was difficult, the default Cauchy prior $r=.707$ was used. Robustness checks are reported in the Supplementary Materials. Bayes factors are always reported testing the alternative hypothesis against the null hypothesis (two-tailed tests: $\mathrm{BF}_{10}$, one-tailed tests: $\mathrm{BF}_{+0}$ ), credibility intervals are provided. 
Overall binding. A Bayesian one-sample t-test testing the null hypothesis that the population mean was equal to zero against the alternative hypothesis that the population mean was not equal to zero was conducted. The test revealed a $\mathrm{BF}_{10}=62.69,95 \% \mathrm{CrI}[-1.3,-.31]$, indicating that the data was 62 times more likely to occur under the alternative than under the null hypothesis. Hence, there was very strong evidence in favor of the alternative hypothesis.

\section{Experiment 2}

Experiment 1 showed that a tactile sensation and a resulting tone are shifted away from each other in temporal perception, paralleling a temporal repulsion effect. It is important to note that participants directly received a tactile sensation on the left or right index finger without any prior knowledge about which finger would be stimulated. Intentional actions, however, are carried out in line with goals (e.g., pushing left causes a high tone), informing the agent which action is selected. In Experiment 2, we therefore explicitly told participants which tactile sensation would occur, thus allowing them to anticipate the identity of the sensation (left or right). If such identify information is central to intentional action and important for the sense of agency, temporal repulsion might decrease or even be reversed, resulting in temporal binding when participants know on which index finger the tactile sensation will occur.

\section{Methods}

\section{Participants and Design}

Thirty-seven (24 females) volunteers with a mean age of 24 years $(M=23.8, S D=$ 4.51) participated in the study in exchange for course credit or monetary reimbursement. All participants gave written informed consent. 
We slightly changed the experimental procedure of Experiment 1 to render the control condition comparable to the new identity cue condition (see below). Therefore, for the sake of proper testing, in Experiment 2 we compared the control condition with an identity cue condition in a within-subjects task setting. Specifically, we used a 2 (stimulus: tactile vs. auditory) x 2 (type of trial: baseline vs. succession) x 2 (condition: control vs. identity) within-subjects design.

\section{Procedure}

The general procedure and the experimental task resembled Experiment 1 . The stimuli were identical. Differences between the experiments are outlined below.

Acquisition phase. Participants completed the same acquisition phase as in Experiment 1. However, to avoid exclusion of participants (see Experiment 1), participants only advanced to the experimental trials after correctly identifying the learned and negating the novel tactile-auditory pairs and were otherwise redirected to the learning trials.

Experimental trials (altered Libet clock trials). In Experiment 2, a second withinsubjects condition was added. Hence, participants completed two conditions in counterbalanced order: a no cue control condition (same as in Experiment 1) and an identity cue condition.

Both conditions consisted of the same four randomized blocks of experimental trials. To familiarize participants with the differences between the conditions, they completed four separate learning trials at the beginning of each condition - one of each type of block (i.e., baseline tactile, a baseline auditory, a succession tactile and a succession auditory trial in sequential order). 
Contrary to Experiment 1, the clock face was framed by two vertical grey columns on the left and the right side of the screen. This was done to integrate the visual cue in the Libet clock task. In the no cue control condition, these columns always remained grey (See Figure 4). In the identity cue condition, one of the columns (left or right) randomly flashed up in white for $100 \mathrm{~ms}$. A left flash indicated a tactile sensation on the left index finger, and a right flash a tactile sensation on the right index finger. The flash occurred at a random moment during the second rotation of the clock hand. Thus, participants were not able to anticipate the temporal onset of the flash. They were told to attend to the identity of the flash (left or right), as this would inform them which index finger would receive tactile stimulation. On baseline auditory trials, no cue was presented because no tactile sensation occurred on those trials. A visualization of the start of a trial is given in Figure 4.

While earlier research showed robust binding effects for fewer trials (Cornelio Martinez et al., 2017, study 1; Moore et al., 2010), in Experiment 2, we increased the number of test trials per block from 20 to 30 (excluding four practice trials) to better control for the influence of excluded or extreme temporal estimates. That is, each participant completed 272 trials in total.

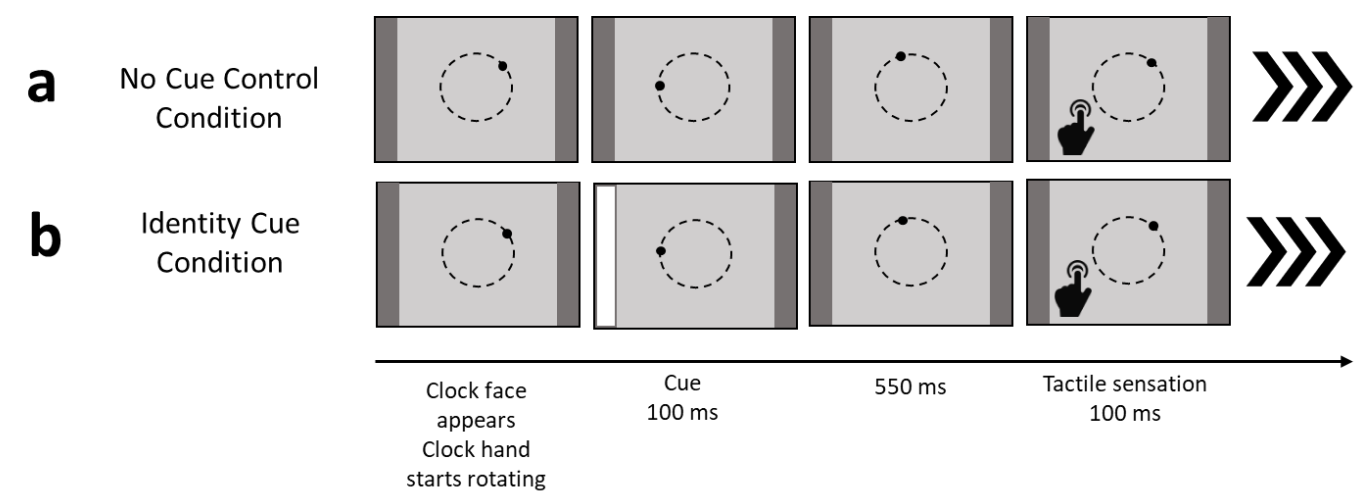

Figure 4. Schematic overview of the start of a trial in two types of conditions: (a) no cue control condition (identical to Exp. 1) and (b) identity cue condition. Identity cues (left or right flash) occurred at a random moment during the second rotation of the clock, followed by the corresponding (left or right) tactile stimulation. In succession trials, tactile stimulation 
was followed by a tone. In baseline trials, only tactile stimulation or a tone was presented. The triple arrows (in bold) indicate the remaining part of a trial, which was identical to Experiment 1.

\section{Data Analysis Plan}

Data preparation and calculation of the dependent variables was the same as in Experiment 1 (see Supplementary Materials for the details). Before the main analysis, we assured that the order in which the conditions were completed did not affect the results. To this end, a repeated measures ANOVA with cue as a within-subjects factor and order as a between-subjects factor was conducted. Next and after collapsing the data, we subjected the overall binding scores to a two-tailed one-sample t-test against zero to test whether the temporal repulsion effect of Experiment 1 was replicated or reversed into binding. Since we added a new condition and only had one prior result, we decided for a two-tailed test as we did not have a clear expectation about the direction (i.e. temporal binding or repulsion) of the effect. Then we conducted a one-tailed paired samples t-test testing our hypothesis that the identity cue attenuates temporal repulsion (indicative of more coherence perception). Finally, we assessed whether temporal binding or repulsion was present in both conditions. Hence, we conducted two-tailed one-sample t-tests against zero using the overall binding scores in the respective conditions.

Additionally, equivalent Bayesian analyses were conducted.

\section{Results}

Effect of order. The repeated measures ANOVA with cue as a within-subjects factor and order as a between-subjects factor showed that neither the main effect of cue, $F(1,35)=$ $3.68, p=.063, \eta_{\mathrm{p}}{ }^{2}=.10$, the main effect of order, $F(1,35)=.01, p=.946, \eta_{\mathrm{p}}{ }^{2}=.00$, nor the 
interaction between cue and order, $F(1,35)=1.52, p=.226, \eta_{\mathrm{p}}^{2}=.04$, was significant and the data was therefore collapsed.

Overall binding. Subjecting the overall binding score to a two-tailed one-sample t-test against zero showed that the overall temporal repulsion effect $(M=-34.78, S D=116.54)$ was not significantly different from zero, $t(36)=-1.82, p=.078, d=-.32,95 \%$ CI $[-.65, .01]$.

Influence of cue. Results of the one-tailed paired samples t-test confirmed that temporal repulsion in the identity cue condition $(M=-19.41, S D=129.31)$ was significantly smaller than in the no cue control condition $(M=-50.15, S D=122.89), t(36)=-1.94, p=$ $.031, d_{z}=-.24,95 \% \mathrm{CI}[-.5, .01]$. Note that this effect equals the three-way within interaction effect between target, type of trial and cue condition in a repeated measures ANOVA, $F(1$, 36) $=3.75, p=.061, \eta_{\mathrm{p}}^{2}=.09$.

Overall binding in the conditions. While the temporal repulsion effect in the no cue control condition was statistically different from zero, $t(36)=-2.48, p=.018, d=-.41,95 \%$ CI $[-.74,-.07]$, this was not the case in the identity cue condition, $t(36)=-.91, p=.367, d=-$ $.15,95 \% \mathrm{CI}[-.47, .17]$. For the distribution of the temporal binding scores in the separate conditions, see Figure 5. 


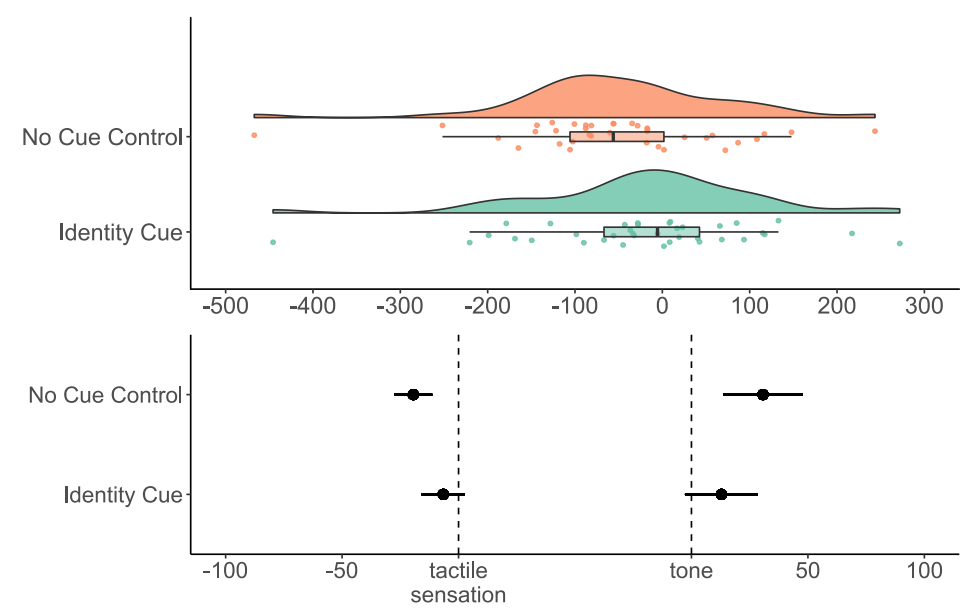

Figure 5. Top: Temporal binding scores in ms per condition in Experiment 2. Dots show the distribution of the individual data points, boxplot summaries use the median. Bottom:

Separate temporal shifts from baseline, dots denote the mean, bars represent standard errors.

\section{Bayesian analysis}

Overall binding. A Bayesian one-sample t-test of the overall binding scores against zero indicated neither evidence for temporal binding nor for temporal repulsion, $\mathrm{BF}_{10}=.79$, $95 \% \operatorname{CrI}[-.59, .05]$.

Influence of cue. A Bayesian paired samples t-test testing the hypothesis that the population mean of the no cue control condition was larger than in identity cue condition resulted in a $\mathrm{BF}_{+0}$ equal to $1.83,95 \% \mathrm{CrI}[-.63,-.04]$, indicating anecdotal evidence for the alternative hypothesis.

Overall binding in the conditions. Two separate Bayesian one-sample t-tests testing the null hypothesis that the population mean is equal to zero against the alternative hypothesis that the population mean is not equal to zero were conducted. For the no cue control condition, the test revealed a $\mathrm{BF}_{10}=2.57,95 \% \mathrm{CrI}[-.71,-.05]$, indicating that the data was 2.5 times more likely to occur under the alternative than under the null hypothesis and therefore anecdotal evidence in favor of the alternative hypothesis. For the estimates in the 
identity cue condition, a $\mathrm{BF}_{10}=.26,95 \% \mathrm{CrI}[-.45, .18]$ and therefore no evidence for the alternative hypothesis given the data in the identity cue condition was found.

\section{Experiment 3}

Experiment 2 tested the robustness of our findings and investigated whether expectations about the identity of a tactile sensation facilitate temporal binding between a tactile sensation and a resulting auditory stimulus. We replicated the temporal repulsion effect and found evidence for an attenuation of the effect when the identity of the tactile sensation was explicitly known in advance. Nonetheless, prior knowledge about the identity of the tactile sensation was not sufficient to cause temporal binding.

In Experiment 3, we added another important component of intentional action, namely the temporal onset of motor action (Brass \& Haggard, 2008). Whereas participants in Experiment 2 were explicitly told to use the identity of the flash as a cue for which tactile stimulation (left or right) would occur, in Experiment 3, we explicitly instructed participants to anticipate the identity and temporal onset of the tactile sensation. To achieve this, the respective identity cue of the tactile sensation (left or right) flashed up in a stable rhythmic pattern, serving as a countdown for the temporal onset of the tactile sensation. This way, participants were not only informed about the identity of the sensation, but they also were able to anticipate the temporal onset of the tactile sensation. If temporal control of motor movement is central to intentional action and shaping a sense of agency, further attenuation of the temporal repulsion effect and even reversal to temporal binding may emerge when participants can anticipate the temporal onset of the tactile sensation. Accordingly, to test this in Experiment 3 we compared the identity cue only condition of Experiment 2 with a new identity + temporal onset cue condition.

\section{Methods}




\section{Participants and Design}

Forty-two (29 females) volunteers with a mean age of 24 years $(M=24.02, S D=6.15)$ participated in this study in exchange for course credit or monetary reimbursement. All participants provided written informed consent.

The experiment used a 2 (target: tactile vs. auditory stimulus) x 2 (type of trial: baseline vs. succession trials) x 2 (cue: identity only vs. identity + temporal onset) withinsubjects design.

\section{Procedure}

The general procedure and the experimental task resembled Experiment 2. Differences between the experiments are outlined below.

Acquisition phase. The acquisition phase was identical to Experiment 2.

Experimental trials (altered Libet clock trials). Participants completed two withinsubjects conditions - an identity cue only condition (identical to the cue condition of Experiment 2) and an identity + temporal onset cue condition - in counterbalanced order.

Each condition consisted of the same four randomized blocks. To familiarize participants with the differences between the conditions, they completed four separate learning trials at the beginning of each condition - one of each type of block (i.e., baseline tactile, a baseline auditory, a succession tactile and a succession auditory trial in sequential order). 
The conditions differed in the cues delivered. In the identity cue condition, the procedure was identical to the one employed in Experiment 2. In the new identity + temporal onset cue condition, one of the columns flashed up white for $100 \mathrm{~ms}$ twice (e.g., left, left) before the tactile stimulation occurred. The time window after each flash was equal $(550 \mathrm{~ms})$, thus forming a clear rhythmic pattern. This enabled participants to use the successively presented cues as a time counter for the onset of the tactile stimulation of the index finger. Accordingly, the identity + temporal onset cue condition not only informed participants which index finger would be stimulated, but also allowed them to more carefully anticipate at which moment in time this stimulation would take place. The cues were only presented on trials in which a tactile sensation occurred (i.e., baseline tactile, succession tactile and succession auditory). Figure 6 displays the start of a trial in both conditions.

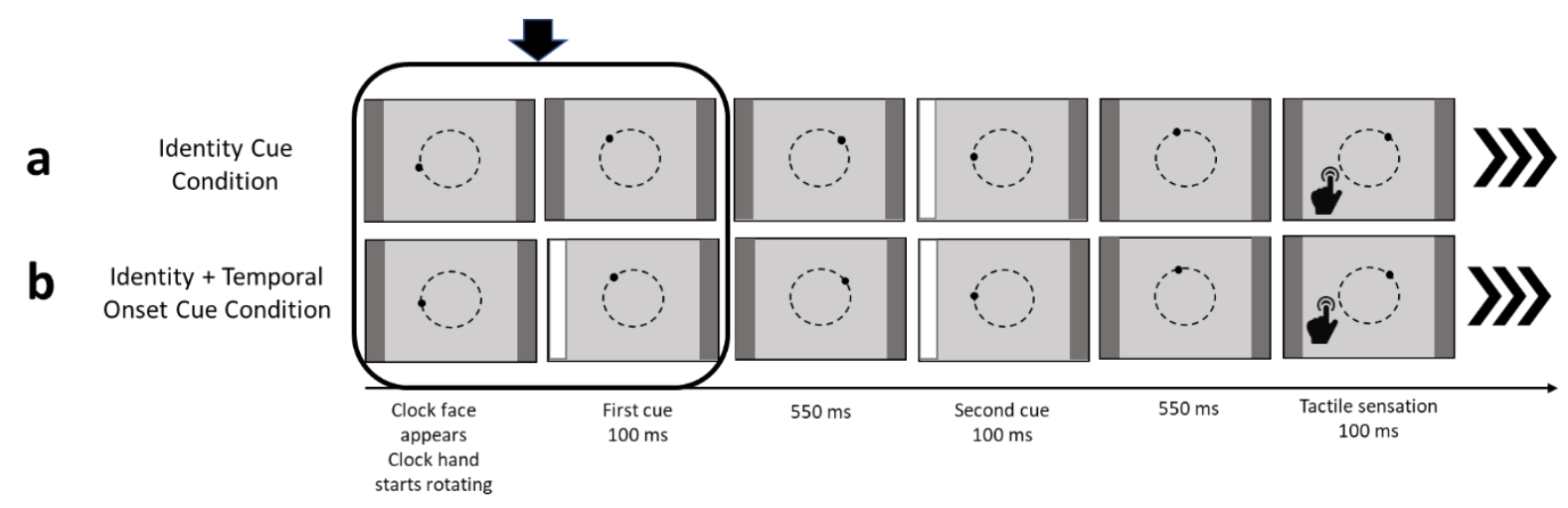

Figure 6. Schematic overview of the start of a trial in the two types of conditions: (a) identity cue condition (identical to Exp. 2) and (b) identity + temporal onset cue condition. In the identity + temporal onset condition, two flashes (e.g., left) occur before the tactile input (left index finger). The time window after the first and second flash is equal (550 ms), thus serving as a countdown procedure for the temporal onset of the tactile stimulation. The rectangle highlights the difference between the beginning of trials in the two conditions. The triple arrows (in bold) indicate the remaining part of a trial, which was identical to Experiments 1 and 2 . 


\section{Data Analysis Plan}

The data of two participants was excluded as technical issues caused inadequate display of the temporal onset cues, resulting in a final sample of 40 participants. Data preparation and calculations of the dependent variables were the same as in Experiment 1 and 2 (see Supplementary Materials for details).

We again first conducted a repeated measures ANOVA on the overall shift scores with cue as a within-subjects factor and order of the conditions as a fixed factor to assure that the order in which the two conditions were presented did not significantly affect participants' judgment errors. We then continued with testing whether temporal repulsion or temporal binding was present in the overall sample. To that end, we conducted a two-tailed one-sample t-test on the overall binding scores against zero. Next, a one-tailed paired-samples t-test was conducted on the shift scores to test if the identity + temporal onset cue helped coherence perception (i.e., weaker temporal repulsion) as compared to the identity cue. Finally, to test whether temporal binding or temporal repulsion was present in both conditions, two-tailed one-sample t-tests against zero were conducted.

As in Experiment 1 and 2, we conducted parallel equivalent Bayesian analysis to assess the strength of the evidence.

\section{Results}

Effect of order. The results of the repeated measures ANOVA on the overall shift scores with cue as a within-subjects factor and order of the conditions as a fixed factor showed no main effect for cue, $F(1,38)=1.57, p=.217, \eta_{\mathrm{p}}^{2}=.04$, no main effect for order, $F(1,38)=3.08, p=.087, \eta_{\mathrm{p}}^{2}=.08$ and no interaction between cue and order, $F(1,38)=1.76$, $p=.193, \eta_{\mathrm{p}}^{2}=.04$. Hence, the data was collapsed. 
Overall binding. The results of the one-sample t-test against zero showed that the mean temporal repulsion effect $(M=-27.88, S D=89.96)$ was not statistically significant, $t(39)=-1.96, p=.057, d=-.31,95 \%$ CI $[-.63, .01]$

Differences between conditions. The one-tailed paired-samples t-test showed that the repulsion effect in the identity cue condition $(M=-36.83, S D=82.21)$ was stronger than the identity + temporal onset cue condition $(M=-18.92, S D=111.59)$, but this was not significant, $t(39)=-1.47, p=.077, d_{z}=-.18,95 \%$ CI $[-.43, .07]$. Note that this effect equals the three-way within interaction effect between target, type of trial and cue condition in a repeated measures ANOVA, $F(1,39)=2.12, p=.154, \eta_{\mathrm{p}}^{2}=.05$.

Overall binding in the conditions. Whereas a significant temporal repulsion effect was found in the identity cue condition, $t(39)=-2.83, p=.007, d=-.45,95 \%$ CI $[-.77,-.12]$, the shift was not significant in the identity + temporal onset cue condition, $t(39)=-1.07, p=$ $.29, d=-.17,95 \%$ CI $[-.48, .14]$. See Figure 7 for a visualization of the temporal binding scores and shifts in the separate conditions.

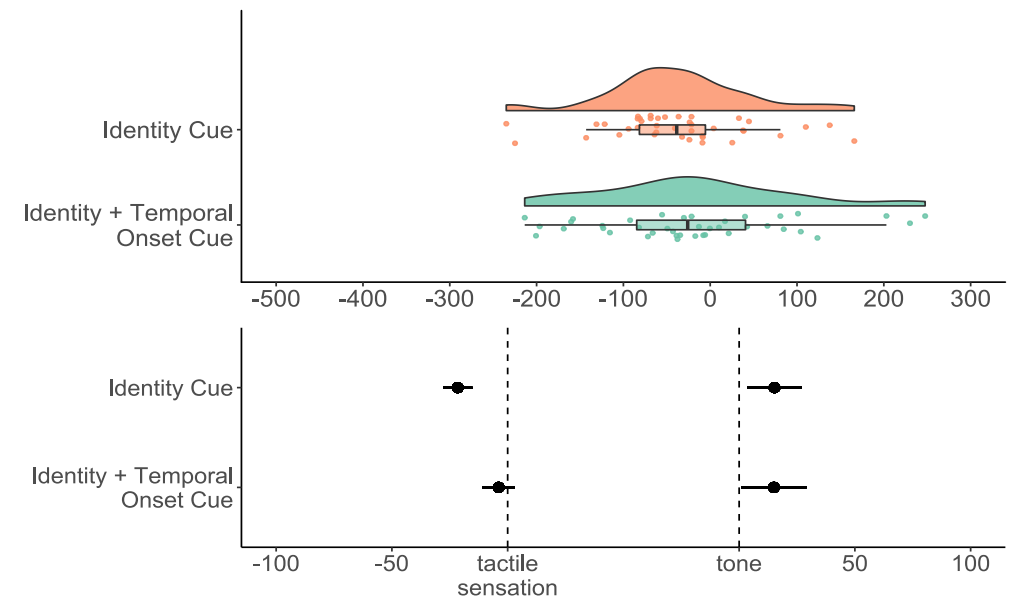

Figure 7. Top: Temporal binding scores in ms per condition in Experiment 3. Dots show the distribution of the individual data points, boxplot summaries use the median. Bottom:

Separate temporal shifts from baseline, dots denote the mean, bars represent standard errors. 


\section{Bayesian analysis}

Overall binding. A Bayesian one-sample t-test of the overall binding scores against the alternative hypothesis of no difference showed no evidence in favor of temporal repulsion, $\mathrm{BF}_{10}=.96,95 \% \mathrm{CrI}[-.6, .02]$

Differences between conditions. A Bayesian paired-samples t-test conducted on the overall shifts scores in both conditions showed no evidence in favor of the alternative hypothesis that there was less temporal repulsion in the identity + temporal onset cue condition than in the identity cue condition over the null hypothesis of no difference, $\mathrm{BF}_{+0}=$ $.83,95 \%$ CrI [-.52, -.02]

Overall binding in the conditions. Separate Bayesian one-sample t-tests on the overall shift scores testing the hypothesis that the population mean does not equal zero were conducted. For the identity cue condition, a $\mathrm{BF}_{10}$ of $5.37,95 \% \mathrm{CrI}[-.73,-.1]$ and thus substantial evidence for the hypothesis that the population mean differed from zero was found. In the identity + temporal onset cue condition, there was no evidence for a deviation of the population mean from zero, $\mathrm{BF}_{10}=.29,95 \% \mathrm{CrI}[-.45, .14]$.

\section{General Discussion}

The present research addressed the question of whether intentional binding as a reflection of the sense of agency occurs in situations where an agent does not engage in motor action. Previous research suggests that temporal binding emerges even when people do not actively engage in goal-directed action. Here, we further explored this issue by examining the role of self-related bodily sensations in temporal binding of such sensations and a resulting effect in the full absence of motor action. Specifically, we delivered tactile input to the fingertip as a haptic sensation that usually accompanies a finger press on a key in order to cause an effect (e.g., an auditory stimulus). If temporal binding occurred, this would 
demonstrate that, in principle, people could experience agency over sensory events that do not result from actual motor movement. Instead of observing temporal binding, we established a robust temporal repulsion effect between a tactile sensation on the fingertip and the auditory effect that resulted from it, showing a dramatic disruption of the sense of agency.

The occurrence of temporal repulsion suggests that a tactile sensation and an auditory effect are separated from each other instead of being integrated into a coherent representation (Desantis, et al., 2012; Haggard et al., 2002a). As previous research on the sense of agency suggests, intentionality and corresponding motor actions are an important amplifier of temporal binding (Antusch et al., 2019; Moore \& Ohbi, 2012; Tanaka \& Kawabata, 2019). Intentional action relies on a sensorimotor control model that is generally assumed to capture the identity and temporal onset of intentional action and its sensory consequences (Blakemore et al., 2002; Haggard, 2017), thus linking them together in the perception of the agent. However, a similar mechanism is absent during passive tactile sensation, even though such sensations are usually part of intentional movement itself. Accordingly, intentional action and passive tactile stimulation differ in the expectation of which stimulus sensation will occur when.

Indeed, our findings indicated that repulsion between a sheer tactile stimulation and an auditory effect was partly corrected when participants were able to anticipate which tactile sensation (on the left or right index finger) would occur and when it would occur. That is, participants perceived more coherence between the tactile sensation and its effect and experienced them as being more temporally related when they were explicitly encouraged to predict the identity and temporal onset of the haptic sensation. This concurs with other research arguing that predictive processes are the primary source that links events together in awareness (Eagleman \& Holcombe, 2002; see also Tanaka et al., 2019). Importantly, although information of the identity and anticipation of temporal onset of the haptic sensation improved 
perceptual binding between tactile sensation and auditory effect, it did not cause temporal binding.

An important question arising from our findings is why we did not find temporal repulsion to be reversed into temporal binding when crucial agency information about the identity and temporal onset was available, especially in light of temporal binding effects obtained in previous work on observation of movement (Suzuki et al., 2019; Vastano et al., 2018). Although we do not have a conclusive answer, a possible difference between our study and the previous research on passive and observed movement lies in the intentional experience of the agent. While participants in former studies might have mentally simulated and even covertly executed the action based on visual input of a moving (virtual) hand and formed intentions based on this, our experimental set-up ruled out motor movement or any information referring to intentional action. Put differently, our participants did not form an intention to produce the tactile sensation and resulting tone but might have held a belief about the causal association between the events (Buehner, 2012), causing a stronger coherence perception but preventing a clear experience of agency. This suggests that holding intentions in mind upon changing bodily sensations and causing effects can increase the perceived temporal attraction between them. The general idea that intentions are important in binding body and environment into a coherent conscious experience is questioned (Dogge et al., 2019; Wegner, 2002), and therefore remains a subject to future empirical scrutiny.

To conclude, we observed that the time-interval between a tactile sensation and an external sensory consequence is subjectively magnified in the absence of motor action, showing weak perceptual coherence between haptic sensations and auditory effects. Our findings suggest that temporal repulsion does not easily reverse into binding that is commonly associated with intentional action - even when a person can predict the identity and temporal onset of body sensations and effects that will occur. Therefore, while our findings point to the 
importance of expectations for integrating tactile sensations and external effects, intentionality seems to have a special status in binding action and effect together and thus might be crucial in shaping a sense of agency and experiences of control when interacting with the external world. 


\section{Chapter 4}

Intentional Action and Limitation of Personal Autonomy. Do Restrictions of Action Selection Decrease the Sense of Agency?

Based on: Antusch, S., Custers, R., Marien, H., \& Aarts, H. (revision resubmitted). Intentional Action and Limitation of Personal Autonomy. Do Restrictions of Action Selection Decrease the Sense of Agency? 


\begin{abstract}
The experience of being an intentional agent is a key component of personal autonomy. Here, we tested how undermining intentional action affects the sense of agency as indexed by intentional binding. In three experiments using the Libet clock paradigm, participants judged the onset of their action (key presses) and resulting effect (auditory stimuli) under conditions of no, partial, or full autonomy over selecting and timing their actions. In all cases, we observed a moderate to strong intentional binding effect. However, we found no evidence for an influence of personal autonomy on intentional binding. These findings thus suggest that being unable to decide how and when to perform actions does not affect the perceived temporal binding between action and effect associated with the sense of agency. We discuss the implications of our findings in the context of research on personal autonomy and goaldirected behavior.
\end{abstract}


Autonomy, i.e. the ability to make unconstrained decisions and to act on them in the service of personal goals, is a core element of democratic constitutions. Maintaining personal autonomy is a central challenge for modern societies characterized by institutional contexts, which allow for unconstrained actions but have (juridical and moral) limitations to them. For example, while individuals are free to move to reach their travel goal, they are expected to follow traffic rules, such as stepping on the brake upon seeing a red traffic light. This mix of intention-based and environment-based action selection highlights the complexity of the concept of personal autonomy. Crucially, personal autonomy is presumed to depend on the sense of agency, i.e., the pre-reflective experience of oneself as the agent of one's actions and their consequences in the external world. The experience of a sense of agency is essential to goal-directed behavior, and hence, to self-regulation. As a more socially reflective construct, the sense of agency also constitutes the basis for causal blaming or judgment of responsibility, and finally, the understanding of moral behavior (Moretto et al., 2011).

Recent research started to explore the relationship between autonomy and the sense of agency. Sense of agency is reflected in intentional actions and their effects being temporally bound together in the perception of the agent, causing the interval between them to be experienced as compressed - a phenomenon termed intentional binding (Haggard et al., 2002b). So far, a set of different personal autonomy restrictions and their influence on binding has been tested, varying from direct brain stimulation of motor movement, to the limitation of behavioral options and coercion (Barlas \& Obhi, 2013; Caspar et al., 2016; Haggard et al., 2002b). While there is considerable literature suggesting that autonomy is a desirable state that is crucial for human performance (Koestner, 2008; Ryan \& Deci, 2006), studies testing the relation between personal autonomy and the sense of agency show mixed results. The present research aims to contribute to this literature by systematically exploring two core components central to personal autonomy and action selection: the ability to decide how to act 
and the ability to decide when to act (Brass \& Haggard, 2008). Specifically, we tested whether the sense of agency, as indexed by the intentional binding effect, reduces when the action selection and action timing shift from being fully autonomous to being fully nonautonomous.

The most prevalent method to assess intentional binding builds on the Libet clock paradigm, in which participants observe a rotating clock while pressing a single key to cause a tone (Tanaka et al., 2019) ${ }^{8}$. Voluntarily pressing a key has been shown to result in the key press and the subsequent effect to be bound together in temporal perception. While the default condition in most intentional binding studies is called 'voluntary action', participants usually can only press a single key and are thus unconstrained in the timing of their action but not the selection. Early findings on involuntary action induction using transcranial-magnetic stimulation (TMS), showed that this binding effect is reversed when autonomy is taken away from the individual, resulting in action and effect to be perceived as repulsed from each other (Haggard et al., 2002b). Also when another agent or a machine induces actions in participants, binding decreases (Borhani et al., 2017; Dogge et al., 2012; Haggard et al., 2002b). Hence, it could be concluded that personal autonomy restrictions - i.e. not being able to act voluntarily - result in an attenuation of the sense of agency. However, not only is external induction of an action an uncommon occurrence in real life, it also denies any opportunity for the agent to form intentions to execute an action, start up the motor system and thus internally generate the action to achieve goals. That is, personal autonomy is not only a function of oneself acting but also characterized by the agent being able to choose the action and the timing of action execution.

\footnotetext{
${ }^{8}$ Note that there is a substantial amount of IB research using the interval estimation method. However, the interpretation of those findings is not always clear as some studies suggest that underestimation equals binding while others interpret overestimation as binding. Whereas both may be true, a more logical way to interpret time interval estimation is to assume that underestimation represents more binding and overestimation more repulsion (see for a discussion on this issue, Antusch et al., 2020).
} 
A first attempt to examine autonomy restrictions in an action selection task is offered by research conducted by Wenke and colleagues (2009). In their study, they used the Libet clock to assess intentional binding and manipulated whether participants could freely choose or were instructed to press one key out of two options (free vs. restricted action selection) and when to press it (free vs. restricted action timing). Findings were that binding magnitude depended on the combined mode of action selection and of action timing. That is, an action that was generated based on two unmatched modes (i.e., internal action selection \& external timing or external action selection \& internal timing) resulted in less intentional binding than when the modes matched (i.e., both internally determined, or both externally determined). The authors speculated that individuals establish a more coherent or fluent experience of behavior when actions result from a single (versus different) mode of selection, thereby increasing binding between action and effect. In other words, an action that is either entirely selfdetermined or entirely externally determined produces an experience of voluntary action. Whereas this metacognitive account might explain the results, it is important to note that participants had to postpone their action, such that they were still able to freely enact their action intention after considerable time (after one rotation of the clock) and within a large time window (during the second rotation of the clock) in the external timing condition. Thus, while suggestive, these findings are not conclusive with regard to the influence of autonomy restrictions on action-effect binding.

In another set of studies, autonomy restrictions were examined in a multiple choice option context. In one study (Barlas et al., 2013), participants were given seven buttons, and they could freely choose which button to press, or they were restricted to three or only one action alternative. They then had to press the button at a self-determined moment while watching the rotation of the Libet clock. Results suggested that intentional binding was strongest when participants had a high level of choice (seven buttons to choose from) as 
compared to a medium level (three buttons) and no choice (one button). Suggesting that maximal autonomy in action selection and timing facilitates a sense of agency, these findings would be in line with related research on ideomotor learning indicating stronger ideomotor learning effects when autonomy is high (Pfister et al., 2011). A possible mechanism herein might be alleviated muscular efficiency due to autonomy support (Iwatsuki et al., 2019).

In a more recent study, Tanaka and Kawabata (2019) adapted the paradigm and compared a high choice condition (free choice out of eight buttons) against a no choice condition (one button) while also manipulating outcome valence. While emotional valence of the action-outcome affected binding (see also Takahata et al., 2012; Yoshie \& Haggard, 2013), the findings of Barlas and colleagues turned out to be difficult to replicate. Although the difficulty to replicate the choice effects on binding might be the result of slight differences in task setting, it is interesting to note that participants in the original study by Barlas and colleagues (2013) did not show binding in the one button (no choice) condition. This is peculiar given that this equals the default 'voluntary action' condition in intentional binding research and usually results in robust binding effects. While this divert finding still needs further clarification, it is conceivable that participants in the study by Barlas et al. might have been more aware of the lack of control or otherwise experienced explicit cognitive reactance because they felt that their freedom was limited. Thus, whereas these studies do not directly compare conditions in which an action is either entirely self-determined or entirely externally controlled, they do suggest that intentional binding could be affected by conscious experiences of restrictions in personal autonomy.

Recent research by Schwarz and colleagues (2019) actually hints at a distinct influence of such autonomy restrictions on explicit agency experiences but not implicit markers such as intentional binding. Using a self-paced Libet clock paradigm in conjunction with an explicit agency measure, they found no difference in intentional binding between instructed and free 
action while the subjective explicit experience of agency differed significantly. Participants reported experiencing less control when they had to select one of two key options but this was not reflected in a weaker binding effect. This finding suggests distinctive mechanisms for the effect of autonomy on the judgment of agency and the feeling of agency (cf. Synofzik et al., 2008). While judgments of agency seem to be affected by explicitly viewing oneself as an agent, feelings of agency as reflected by intentional binding seem to show a more implicit experience of agency. This also explains why agents who only observe an action but do not execute it report no explicit agency but show intentional binding (Obhi \& Hall, 2011). This leads to the question of whether full autonomy restrictions in choosing and timing an action affect implicit experiences of agency at all.

Research on coercion, in contrast, suggests that autonomy restrictions can decrease the implicit sense of agency (Caspar et al., 2016; 2017). In a study by Caspar and colleagues (2016), participants could either freely choose to inflict financial or physical pain on another participant or were coerced to do so by the experimenter. It was shown that participants showed less temporal binding when they executed an action based on the experimenter's "command" as compared to when they freely chose to do so. Note however that inflicting financial or physical pain on another person is a strong manipulation of the action-outcome valence, which might be stressful. Moreover, participants benefitted financially (i.e., they earned money for every time that they inflicted pain) when they inflicted pain on another agent, which might have confounded results. Furthermore, rather than using the Libet clock, the coercion studies asked participants to estimate the time interval while action timing was self-determined. This makes the findings somewhat difficult to interpret in the context of previous work on the role of personal autonomy restrictions in the mere stage of action selection and timing on binding in the Libet clock task. 
To summarize, the existing literature applied a range of different autonomy restrictions to examine how having control over the action selection process affects the sense of agency. Autonomy restrictions mainly applied to the choice options and the moment of acting. While both aspects are important, the moment of action selection is easily confounded with external control of action. That is, in previous studies participants were told when to press, but they still had sufficient freedom to select and time their action within a large time window. In the present study we therefore aimed to scrutinize freedom of action timing in a more strict sense. Specially, we examined the situation in which participants are told when to exactly press a key, namely when a specific cue would appear on a computer screen. This way, freedom of action timing is narrowed down to a more environment-controlled action selection process and thus rules out the ability to voluntarily time the action.

To systematically examine the role of autonomy with respect to choice and timing in the sense of agency we designed three experiments using the Libet clock task. In the first experiment, we developed a task similar to the one used by Schwarz et al. (2019), and measured the strength of intentional binding when participants are forced to select one out of two action options. The timing of action selection was free. Experiment 2 offers a test that compares the effects of partial autonomy (forced choice, free timing) and full personal autonomy (free choice, free timing) of action selection on the sense of agency. In Experiment 3 we compared partial autonomy with no autonomy during action selection by also restricting the timing of action. That is, participants were required to press a specific key immediately when they encountered a specific cue.

\section{Experiment 1}

\section{Methods}

\section{Participants and Design}


Twenty-nine (18 females) volunteers with a mean age of 22 years $(M=22.38, S D=$ 2.4) participated in the study in return for course credit or a financial reimbursement. All participants gave written informed consent.

The experiment used a 2 (target: action vs. tone) x 2 (type of trial: baseline vs. operant) within-subjects design. All experiments were approved by the Ethics Review Board of the Faculty of Social and Behavioral Sciences, Utrecht University as part of a project line (FETC approval code: 19-018). Power analyses for all experiments are reported in the Supplementary Materials.

\section{Procedure}

Participants were seated in front of a computer screen in a separate cubicle. After written informed consent was obtained, participants put on the headphones and began with the experiment. All instructions were provided on screen. The experiment was programmed in Eprime 2.0 and consisted of an adapted Libet clock task.

Two actions and two auditory stimuli were used in the experiment. The actions were a $x$-key press (left) and a $n$-key press (right) on a QWERTY keyboard. The auditory stimuli consisted of a low (300 Hz frequency) and a high (1000 Hz frequency) sinusoidal tone of 100 ms length each.

Learning phase. The experiment began with a learning phase to familiarize participants with the two actions and the two auditory stimuli. After an initial presentation of the key presses and the tones, the tones were repeated ten times in random order (i.e., five trials per tone) to allow participants to become acquainted with them. Subsequently, participants learned that each key was paired with one of the tones. Hence, each participant learned two key-tone pairs. The combination of the key-tone pairs (i.e., left-key and $1000 \mathrm{~Hz}$ and right-key and $300 \mathrm{~Hz}$ or left-key and $300 \mathrm{~Hz}$ and right-key and $1000 \mathrm{~Hz}$ ) were 
counterbalanced across participants but remained stable per participant. In ten trials, participants pressed one of the keys, which was then followed by the paired tone.

Finally, participants were presented with the two learned as well as two novel (i.e., obtained by pairing a key with the tone of the respective other pair) key-tone pairs and had to correctly identify the learned and negate the novel pairs. Incorrectly answered trials were repeated. Participants could only commence with the experimental trials after correctly answering all trials.

Experimental trials (altered Libet clock trials). The experimental task was an altered Libet clock task as is conventionally used for assessing intentional binding (see also Antusch et al., 2019; 2020). Participants completed four randomized blocks (i.e., two baseline and two operant blocks). Each block consisted of 20 experimental trials and five practice trials, resulting in a total of 100 trials per participant. Practice trials were indistinguishable from experimental trials but were discarded for the statistical analysis.

On each trial participants viewed a clock face that consisted of 40 grey dots, arranged in a circle from the center with a diameter of six $\mathrm{cm}$. A black dot that moved at a speed of $2560 \mathrm{~ms}$ per rotation served as the clock hand. The initial position of the clock hand was determined randomly. Depending on the block, either one event (baseline blocks) or two successive events (operant blocks) occurred. On baseline blocks, participants either only pressed a key (baseline action) or heard a tone (baseline effect) while viewing the clock face and clock hand. In operant blocks, participants first pressed a key, which was followed by the paired tone $250 \mathrm{~ms}$ later. On all trials involving an action (all except for the baseline tone block), a visual brief message was presented in the center of the screen for $3000 \mathrm{~ms}$ before the clock face was presented. This message indicated which key (x or n) participants should press on a given trial, thus restricting participants in their choice of action options (Schwarz et al., 
2019). Left and right key presses were distributed equally across trials (i.e., ten trials per key and block). Participants were instructed to wait with pressing the key until the clock hand had completed one full rotation. Thus, timing of action selection was free. In baseline effect trials, one of the tones was played at a randomly determined moment during the second rotation of the clock hand. Tones were distributed equally across trials (ten trials per tone).

The clock hand rotated further for $1000 \mathrm{~ms}$ after the last event before she disappeared. Subsequently, the mouse cursor and a prompt appeared in the center of the clock face. Participants' task was to indicate when the key press (baseline action and operant action trials) or the tone had occurred (baseline effect and operant effect trials). Participants gave their judgments by clicking on one of the dots of the clock face with the mouse cursor. Then, a new trial started. The inter-trial interval was equal to $1000 \mathrm{~ms}$. See Figure 1 for a visualization of an operant trial.

At the end of the experiment, participants provided their demographics, were thanked for their participation, reimbursed and debriefed.

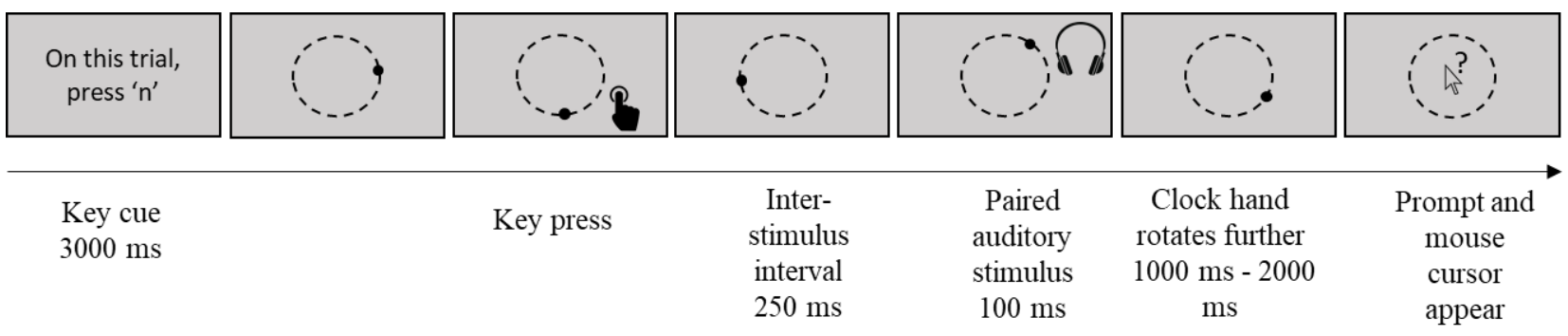

Figure 1. Visualizations of an operant trial. The trial depicts a right key press (i.e., participants had to press the n-key).

\section{Data analysis plan}

Prior to the main analysis, temporal judgments more than one quarter $(+/-640 \mathrm{~ms})$ away from the actual temporal onset were excluded from data analysis (.43\% of all trials; Aarts et al., 2012). Furthermore, judgments on trials in which participants pressed the wrong 
key or pressed a key multiple times were excluded (.69\% of all trials). Overall, the amount of trials excluded based on these criteria was small (1.02\% of trials).

Based on the temporal judgments, mean estimates per block and participant were calculated. Subsequently, using these mean estimates, separate temporal shift scores for the action and the tone shift were calculated by subtracting the mean estimates of baseline trials from the mean estimates of operant trials (action shift = mean estimate in operant action trials - mean estimate in baseline action trials; tone shift = mean estimate in operant tone trials mean estimate in baseline tone trials). Finally, overall binding scores were computed by subtracting the tone shift from the action shift (i.e., overall score $=$ action shift - tone shift). Positive overall binding scores thus indicated temporal compression of the interval between action and effect (temporal binding) while negative scores were indicative of temporal repulsion. To test if intentional binding occurs in a forced action context, we subjected the resulting overall binding scores to a one-tailed one-sample t-test.

\section{Results}

The overall binding score $(M=64.58, S D=72)$ was subjected to a one-tailed one sample t-test against zero. The results revealed a strong overall binding effect, $t(28)=4.83, p$ $<.001, d=.9,95 \%$ CI $[.46,1.32]$.

\section{Experiment 2}

In the first experiment, we tested a new task in our laboratory to assess the strength of intentional binding in a multiple action task where participants are forced to press one of two keys, and established that perceptual compression of the temporal interval between action and effect clearly emerged. Being ensured about the validity of our task, we set up a follow-up experiment aimed to replicate the findings obtained in the study of Schwarz et al. (2019). Specifically, participants had either partial autonomy (forced choice, free timing) or full 
autonomy (free choice and free timing) in action selection. Schwarz et al. (2019) found no difference between these two conditions on intentional binding, thus supporting a null effect. Accordingly, we anticipated no difference between partial and full autonomy on intentional binding. Because of the expected null effect, we tested our hypothesis using both, frequentist statistics and Bayesian, statistics.

\section{Methods}

\section{Participants and Design}

Thirty-nine (29 females) volunteers with a mean age of 24 years $(M=23.56, S D=$ 4.29) participated in this study in exchange for course credit or a financial reimbursement. All participants provided written informed consent.

A 2 (target: key press vs. tone) x 2 (type of trial: baseline vs. operant) x 2 (autonomy: partial autonomy vs. full autonomy) within-subjects design was used.

\section{Procedure}

Participants were seated in front of a computer screen in a separate cubicle. After written informed consent was obtained, participants put on the headphones and began with the experiment. All instructions were provided on screen. The experiment was programmed in Eprime 2.0.

Learning phase. The experiment began with the same learning phase as in Experiment 1.

Experimental trials (altered Libet clock trials). Participants completed two withinsubject conditions in counterbalanced order: the partial autonomy condition (resembling Experiment 1) and the full autonomy condition. Each condition consisted of the same four 
randomized blocks (baseline action, baseline effect, operant action and operant effect). Each block consisted of 25 trials (20 experimental plus five practice trials), resulting in 200 trials in total. The general set-up of trials and the parameters of the clock were identical to Experiment 1, differences are outlined below.

Different to Experiment 1, in the partial autonomy condition, when participants pressed a different key than the instructed key, the trial was aborted prematurely, and they received error feedback stating that they had pressed the wrong key to enforce the instructions. In the full autonomy condition, participants could freely choose which key to press and when to press it. Instead of receiving a cue which key to press on a trial, they read a reminder that they could press either of the keys. They were asked to try pressing each key equally often. A setup of a partial and full autonomy trial is depicted in Figure 2.

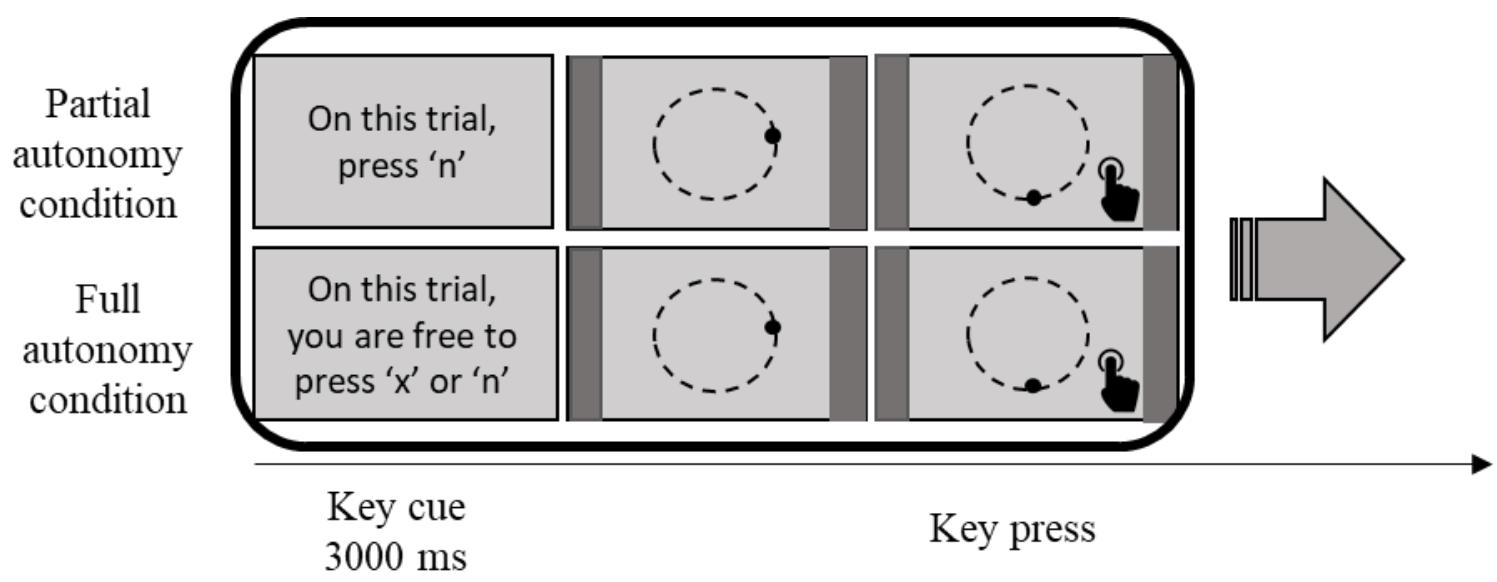

Figure 2. Operant trials in the partial and full autonomy condition. The arrow indicates that the trial in the full autonomy condition proceeded identical to the partial autonomy condition.

\section{Data analysis plan}

As before, prior to the main analysis temporal judgments that were more than one quarter (+/-640 ms) away from the actual temporal onset were excluded from data analysis (Aarts et al., 2012). Furthermore, also trials on which more than one key press was registered 
were excluded. Overall, the amount of trials excluded based on these criteria was small and very similar to the first experiment ( $1.52 \%$ of trials).

Subsequently, mean estimates per block and separate temporal shift scores for the action and the tone were calculated. Shift scores in both conditions were calculated using the same formula as in Experiment 1. Finally, overall binding scores for the separate conditions and for both conditions together were computed by subtracting the tone shift from the action shift (i.e., overall score $=$ action shift - tone shift). Positive overall binding scores thus indicated temporal compression of the interval between action and effect (temporal binding) while negative scores were indicative of temporal repulsion.

After data preparation, we examined whether order of condition affected the intentional binding effects of the partial and full autonomy conditions. Next, for the sake of comparison with findings of Experiment 1, we assessed whether overall binding scores as well as the binding scores in the respective conditions were significantly different from zero. To that end, we conducted three one-tailed one-sample t-tests. Finally, we tested the main hypothesis with a two-tailed paired samples t-test and report whether temporal binding was different in the partial and full autonomy conditions. Additionally, to illustrate the strength of the evidence for the Ho and to confirm the frequentist analyses, equivalent Bayesian analyses were performed. A Bayesian paired samples t-test was conducted using JASP (JASP Team, 2019). As we had no knowledge about a possible prior distribution and the expected strength of the evidence, we chose to use the default Cauchy prior equal to .707. See Supplementary Materials for the robustness check.

\section{Results}

Influence of order. To rule out that the order in which participants completed the two within-subject conditions significantly influenced their temporal perception, we conducted a 
repeated measures ANOVA on the overall binding scores using choice condition as a withinsubject factor and order as a between-subject factor. The results revealed no significant effects: main effect of choice condition, $F(1,37)=.059, p=.809, \eta 2=.00,95 \%$ CI $[.00, .06]$ and interaction between choice condition and order, $F(1,37)=1.374, p=.249, \eta 2=.04,95 \%$ CI $[.00, .20]$.

Overall binding. The one-tailed t-test on the overall binding score revealed a significant temporal binding effect $(M=70.08, S D=108.83), t(38)=4.021, p<.001, d=.64$, $95 \%$ CI $[.29, .99]$.

Binding in the separate conditions. To test if temporal binding emerged in both conditions, two separate one-tailed one-sample t-tests were conducted. Results revealed significant temporal binding in both conditions: partial autonomy condition $(M=68.76, S D=$ 115.96), $t(38)=3.703, p<.001, d=.59,95 \%$ CI $[.25, .93]$ and full autonomy condition $(M=$ 71.39, $S D=114.95), t(38)=3.878, p<.001, d=.62,95 \%$ CI $[.27, .96]$.

Difference between conditions. A paired-samples t-test was conducted to assess the difference between partial and full autonomy conditions. The analyses showed that the difference between the partial autonomy condition $(M=68.76, S D=115.96)$ and the full autonomy condition $(M=71.39, S D=114.95)$ was not statistically significant, $t(38)=-.212$, $p=.833$, two-tailed, $d z=-.03,95 \%$ CI $[-.23, .19] .{ }^{9}$

Finally, Bayesian paired-samples t-test revealed a $\mathrm{BF}_{01}$ equal to 5.627, 95\% $\mathrm{CrI}[-.33$, .27], indicating considerable evidence for the null hypothesis.

\footnotetext{
${ }^{9}$ We inspected the data to see whether participants pressed the left or right key equally in the full autonomy condition (see Supplemental Materials). Only a few participants showed an inclination to (freely) press the same key more than $80 \%$ of the time. Excluding these participants did not change the results: The difference between partial autonomy $(M=51.07, S D=104.05)$ and full autonomy $(M=58.99, S D=110.66)$ was not significant, $t(33)=-.584, p=.563$, two-tailed, $d z=-.10,95 \%$ CI $[-.32, .18]$.
} 


\section{Discussion}

In Experiment 2, we tested how having participants freely select and time their action versus forcing them which action to execute but letting them freely decide on the timing affects intentional binding. Overall, we replicated the intentional binding effect found in Experiment 1. However, our results did not show an increase binding when participants were fully free in their choice over action selection and action timing. This null effect replicates earlier work (Schwarz et al., 2019), and was supported by frequentist tests and Bayesian tests. While our task differs from the task used by Schwarz et al. on a few features (e.g., different keys, different tones, different Libet clock), the absence of a difference appears rather robust. Apparently, then, having partial or full autonomy over action selection does not matter for peoples' sense of agency and the temporal perception between their actions and effects associated with them.

\section{Experiment 3}

So far, in the first two experiments action timing was free. Participants were able to enact their intended action at a self-chosen moment during the second rotation of the Libet clock. Note that such a self-chosen moment of action selection is typical in intentional binding studies (e.g., Haggard et al., 2002b; Moore \& Haggard, 2008; Ruess et al., 2017), suggesting that participants normally have at least partial autonomy. In Experiment 3 we therefore challenged participants' personal autonomy further by forcing them to press a specific key at a specified moment, namely when they encounter a cue on the screen. This way, autonomy over action selection is absent, as the type and moment of action execution is fully delegated to the environment. If personal autonomy is important for the sense of agency, then having no autonomy at all should reduce intentional binding compared to the conventional condition of partial autonomy in intentional binding research. 


\section{Methods}

\section{Participants and Design}

Forty-one (30 females) volunteers with a mean age of 21 years $(M=21.46, S D=2.7)$ participated in this study in exchange for course credit or a financial reward. All participants provided written informed consent.

A 2 (target: key press vs. tone) x 2 (type of trial: baseline vs. operant) x 2 (autonomy: partial autonomy vs. no autonomy) within-subjects design was used.

\section{Procedure}

Participants were seated in front of a computer screen in a separate cubicle. After providing written informed consent, they put on the headphones and began with the experiment. All instructions were provided on screen. The experiment was programmed in Eprime 2.0.

Learning phase. The experiment began with the same general learning phase as was used in Experiments 1 and 2.

Experimental trials (altered Libet clock trials). Participants completed two counterbalanced within-subjects conditions: a partial autonomy condition (same as in Experiments 1 and 2) and a no autonomy condition. Each condition comprised the same four randomized blocks of 25 trials each (20 experimental and five practice trials). Trials and parameters of the clock resembled those in Experiments 1 and 2, and differences are outlined below.

In contrast to the first two experiments, we integrated the visual cue for action timing in the two vertical grey columns on the left and right side of the computer screen. Thus, in the 
new no autonomy condition, participants were not only instructed which key to press but also when exactly to press, namely at the moment that they saw the timing cue. The timing cue consisted of one of the vertical columns on the left of the right side of the screen flashing up in white for $100 \mathrm{~ms}$. The location of the cue (left or right) coincided with the instructed key (i.e., the left column flashed-up when the $\mathrm{x}$-key should be pressed and vice versa for the nkey). Participants were instructed to press the key as soon as possible after the presentation of the cue. If participants pressed the wrong key or pressed before the presentation of the visual cue, the trial was aborted and they received error feedback.

As before, on operant trials the key press was followed by the paired tone at $250 \mathrm{~ms}$. The clock hand then kept rotating for a random time interval between 1000 and $2000 \mathrm{~ms}$ before disappearing. Finally, participants estimated the temporal onset of either the key press or the tone and a new trial began. See Figure 3 for a visualization of operant trials in the two conditions.

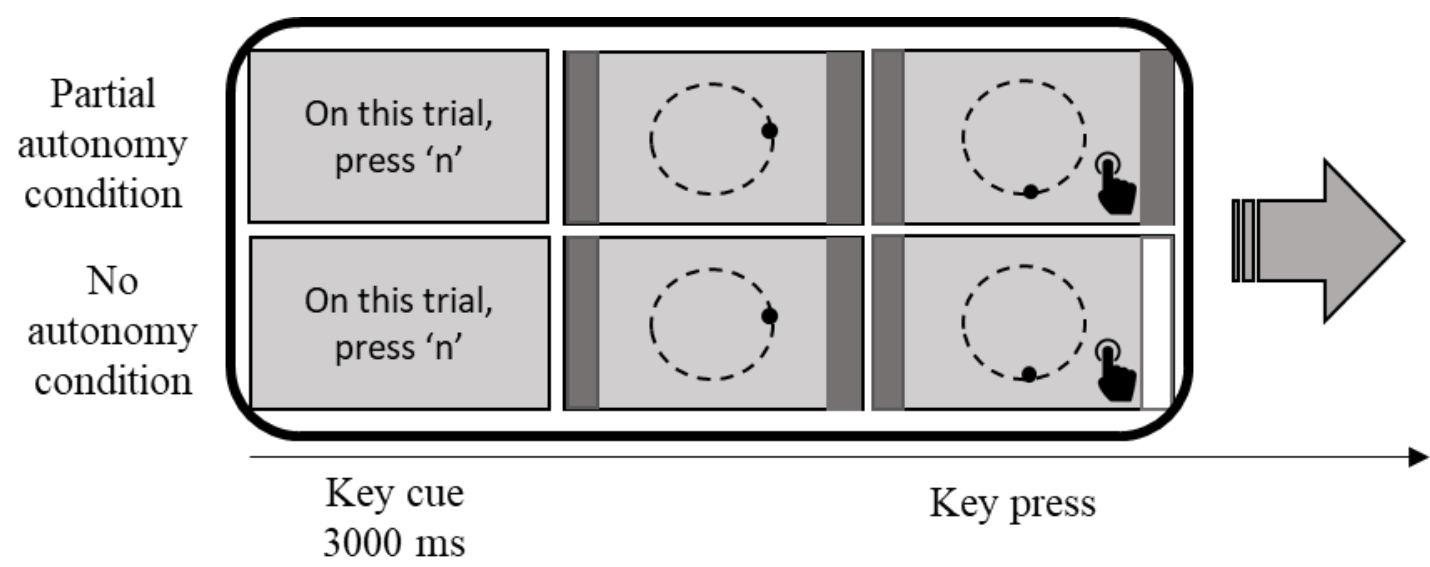

Figure 3. Visualization of an operant trial in the partial autonomy condition and no autonomy condition. The timing cue of in the no autonomy condition is presented in white as part of the vertical column. The arrow indicates that the trials in both conditions proceeded in identical manner. 


\section{Data analysis plan}

Like in the preceding experiments, excessive temporal judgments were excluded from the main analysis (.20\% of all trials). Moreover, trials on which participants pressed the wrong key or reacted too fast (before the presentation of the cue) or too slow (more than three standard deviations away from their mean reaction time) to the visual cue in the forced condition, were set to missing and excluded from data analysis (.99\% of all trials). The data of one participant who reacted slower (more than three standard deviations away from the group mean) than the average of the sample was entirely excluded from analysis. Mean estimates per block, separate temporal shift scores for the action and the tone, binding scores in the two within-subject conditions as well as overall binding scores were calculated using the same formulas as in Experiments 1 and 2.

Next, we performed a few checks. First, we examined whether order of condition affected the intentional binding effects of the partial and full autonomy conditions. ${ }^{10}$ We then tested whether the overall binding scores as well as the binding scores in the respective conditions were significantly different from zero. We therefore conducted three one-tailed one-sample t-tests. Finally, we tested whether temporal binding was different in the control partially instructed and the fully restricted condition with a two-tailed paired samples t-test. To assess the strength of the evidence, an equivalent Bayesian paired-samples t-test was conducted using JASP (JASP Team, 2019). We again used a Cauchy prior equal to .707. A robustness check is reported in the Supplementary Materials.

\footnotetext{
${ }^{10}$ In the no autonomy condition we checked the speed of responding to the timing cue to ensure that participants did not wait too long but indeed pressed the key upon seeing the cue. For each participant we averaged response times across the three blocks that required participants to press an instructed key. On average, participants reacted to the cue in under $600 \mathrm{~ms}(M=578.55)$, indicating that they responded fairly fast to the timing cue in the Libet clock set-up. There were no differences on reaction times between the three blocks, $F(2,38)=.449, p$ $=.642, \eta^{2}=.02,95 \%$ CI $[.00, .14]$.
} 


\section{Results}

Influence of order. To rule out that the order in which participants completed the two within-subject conditions significantly influenced their temporal perception, we conducted a repeated measures ANOVA on the overall binding scores using choice condition as a withinsubject factor and order as a between-subject factor. The results revealed no significant effects. Neither the main effect of choice condition, $F(1,38)=.277, p=.602, \eta 2=.01,95 \%$ CI $[.00, .13]$ nor the main effect of order, $F(1,38)=.028, p=.869, \eta 2=.00,95 \%$ CI $[.00$, $.03]$ nor the interaction between choice condition and order, $F(1,38)=1.392 p=.245, \eta 2=$ $.04,95 \%$ CI $[.00, .20]$, was significant.

Overall binding. To test whether participants showed an overall intentional binding effect (regardless of condition), we subjected the overall binding score to a one-tailed t-test against zero. Results revealed that the overall temporal binding of about $55 \mathrm{~ms}(M=54.64$, $S D=83.41)$ was highly significant, $t(39)=4.143, p<.001, d=.66,95 \%$ CI $[.31, .99]$.

Binding in the separate conditions. To test if temporal binding emerged in both conditions, two separate one-tailed one-sample t-tests were conducted. Results revealed significant temporal binding in both conditions: partial autonomy condition $(M=49.63, S D=$ 99.27), $t(39)=3.162, p=.0015, d=.5,95 \% \mathrm{CI}[.17, .83]$ and no autonomy condition $(M=$ $59.66, S D=127.04), t(39)=2.970, p=.0025, d=.47,95 \%$ CI $[.14, .79]$.

Difference between conditions. A paired-samples t-test was conducted to assess the statistical significance of the difference between the extent of temporal binding in the withinsubject conditions. The results suggested that the difference of roughly $10 \mathrm{~ms}(M=10.03, S D$ $=155.42$ ) between the conditions was not statistically significant, $t(39)=-.408, p=.685$, twotailed, $d z=-.06,95 \%$ CI $[-.51, .36]$. A parallel Bayesian paired samples t-test was conducted 
to assess the strength of the evidence. The analysis revealed a $\mathrm{BF}_{01}$ equal to $5.42,95 \% \mathrm{CrI}$ [$.36, .24]$, indicating considerable evidence for the null hypothesis.

\section{Discussion}

In Experiment 3, we aimed to test whether further reduction of personal autonomy would decrease intentional binding. Therefore, participants were not only instructed to press a specific key (like in the partial autonomy condition), but they also had to press at a designated moment in time, i.e. immediately when seeing an action timing cue. Both conditions of partial and no autonomy produced intentional binding effect and the strength of the effect did not differ. This null effect was corroborated by frequentist tests and Bayesian tests, suggesting that the absence of difference is likely not due to a Type 2 error. Thus, our findings suggest that even assigning the timing of action selection by the environment does no undermine the sense of agency, an effect that seems peculiar in light of the presumed importance of action choice and timing in research on personal autonomy and voluntary action. For a complete overview of the separate overall binding scores in each experiment and condition, please see Figure 4. 


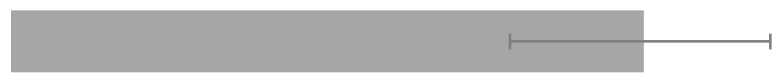

partial autonomy

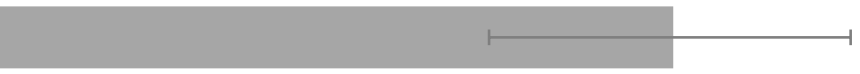

full autonomy

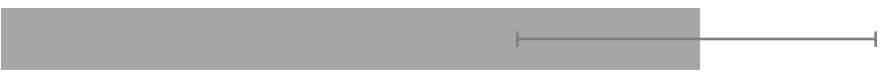

$\stackrel{m}{\frac{1}{0}}$

partial autonomy

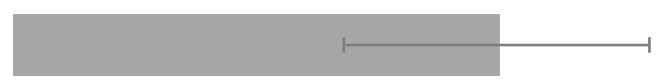

no autonomy

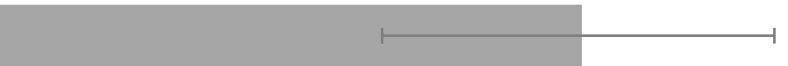

$0 \quad 20$

40

60

80

Figure 4. Overall binding scores in ms per condition for Experiments 1-3. Error bars represent the individual standard errors.

Before concluding that personal autonomy does not affect the sense of agency over producing effects by own actions, as measured by the intentional binding task, we assured that the different objective (full, partial vs. no) autonomy conditions also install different psychological states in line with our manipulations. To this end, we conducted an additional study. We tested the operant blocks of the three respective conditions used in Experiments 1-3 (full, partial and no autonomy) and asked participants to report their subjective experiences of autonomy in each condition. It may be expected that subjective experiences of autonomy over producing effects by own actions decrease when moving from full to partial and to no autonomy.

\section{Extra Check: The relation between objective and experienced autonomy}

\section{Methods}




\section{Participants and Design}

$27^{11}$ (20 females) volunteers with a mean age of 24 years $(M=23.89, S D=3.95)$ took part in the experiment in exchange for monetary reimbursement. The experiment used a 3 (condition: control vs. free vs. forced) x 3 (autonomy parameter: control vs. choice vs. intentionality) within-subjects design.

\section{Procedure}

The task started with the general learning phase (Experiments 1-3). Next, participants completed two blocks: the operant action and the operant effect Libet clock trials, counterbalanced in order. Thus, each trial comprised a key press and a resulting tone. Because we were interested in the psychological experience of producing effects by one's own actions and not temporal perception, the experiment did not use baseline blocks. Each of the two operant blocks comprised three sub-blocks, also counterbalanced in order. The three sub-blocks corresponded to the three types of conditions: full, partial and no autonomy. Participants completed six trials in the operant action block and the operant effect block, resulting in a total of 36 trials (12 for each of the three autonomy conditions).

Similar to Experiments 1-3, in each trial the participants saw the clock face framed by two grey columns on the left and right of the screen. The trial then proceeded, depending on the condition (full, partial or no autonomy). At the end of each trial, participants answered three questions about their experience of autonomy: They indicated how much control (9point Likert scale; 1 - no control , 9 - a lot of control), how much choice (9-point Likert scale; 1 - no control, 9 - a lot of control) and how much intentionality (9-point Likert scale; 1- not

\footnotetext{
${ }^{11}$ We originally aimed for a sample of 30 participants, however, the data of three participants was lost due to technical problems.
} 
at all, 9 - a lot) they experienced on a given trial. In sum, each item was measured 12 times in each condition.

At the end, participants filled in their demographics, were thanked and reimbursed for their participation.

\section{Data analysis plan}

Per condition, for each participant we averaged the 12 control, 12 choice and 12 intentionality items, separately. We then averaged the means for the three items into a measure of subjectively experienced autonomy for each condition (internal consistency Cronbach's alpha - was $.82, .84$ and .92 for the full, partial and no autonomy conditions, respectively. Subsequently, the subjective measure of autonomy was subjected to a repeated measures ANOVA with condition (full, partial, or no autonomy) as within-subject variables. Pairwise comparisons were analyzed to establish which conditions differed from each other.

\section{Results}

The repeated measures ANOVA revealed a significant main effect of condition, $F(2$, $25)=23.849, p<.001, \eta^{2}=.91,95 \%$ CI $[.24, .94]$. Pairwise comparisons showed that the subjectively experienced autonomy in the full autonomy condition $(M=7.76, S D=1.15)$ was significantly higher than in the partial autonomy condition $(M=7.03, S D=1.24), p<$ .001 , and in the no autonomy condition $(M=4.82, S D=2.14), p<.001$. These findings show that the objective situation of full autonomy was experienced as having autonomy, while the objective situation of no autonomy substantially weakened the conscious experience of personal autonomy. Partial autonomy fell in between.

\section{General Discussion}


The present study aimed to address the relationship between personal autonomy and the sense of agency over causing one's actions and their effects in the external world. Specifically, we examined whether and how objective conditions of personal autonomy that undermine a person's intention affect intentional binding when the selection and timing of goal-directed action is forced or free. We thus compared conditions of no autonomy (forced action selection and timing), partial autonomy (forced action selection and free timing) and full autonomy (free action selection and timing). Our findings indicate that intentional binding was equally strong in all three conditions, suggesting that personal autonomy is unrelated to the sense of agency. Importantly, the three autonomy conditions clearly differed on subjectively experienced personal autonomy, showing that our participants were aware of the autonomy restrictions implemented in the task. These findings concur with recent work by Schwarz et al. (2019), who showed that full and partial autonomy significantly differed on explicit measures of control, but not intentional binding as assessed with the Libet clock. The present study extends this work by showing that no autonomy does reduce the conscious experience control further without affecting intentional binding.

Our findings seem to contradict other research on forced and free action selection and timing. Wenke and colleagues (2009), for example, found reduced intentional binding when participants had partial autonomy compared to no or full autonomy. They offered a metacognitive account to explain these effects, according to which individuals establish a more fluent experience of behavior when actions result from a single (versus different) mode of selection. While this account seems plausible, a closer look at the experimental procedure suggests a crucial difference with our procedure. Specifically, in the no autonomy condition participants were instructed to select one key response over the other, but were instructed to press the key somewhere in the second rotation. In other words, participants were forced to press the key in a specified time window but had to wait and then press the key at a preferred 
moment in time. Accordingly, action timing in this condition could be construed as free rather than forced, resembling our partial autonomy condition that is typical for most intentional binding research (e.g., Haggard et al., 2002b; Moore \& Haggard, 2008; Ruess et al., 2017). Furthermore, when action selection was forced and timing was free (identical to our partial autonomy condition), Wenke and colleagues found less binding compared to when having full autonomy of action selection and timing, which differs from our results. As such, our findings concur with those of Schwarz et al. (2019), who did not find differences in intentional binding between partial and full autonomy. These puzzling findings raise the question whether autonomy is relevant for the sense of agency.

To answer this question, we might need to consider the study of personal autonomy in the context of the bigger picture of intentional binding research. First, it is important to note that the forced timing component in our no autonomy condition was operationalized as an environment-controlled action that leaves not much room for free timing, as participants had to immediately respond to a timing cue. Whereas this externally induced action timing did not affect intentional binding, other studies seem to do. For example, initial research on intentional binding comparing free action timing with involuntary action induced by TMS, demonstrated that taking autonomy away from the individual also eradicates the sense of agency, resulting in temporal repulsion instead of binding. In line with this, we found additional evidence for repulsion effects when participants received action-related sensory input (tactile stimulation on the finger that will cause a predicted effect), ruling out the possibility to internally select and execute the action (Antusch et al., under review). Slightly different results are reported for the actual execution of actions that are externally induced by pressing the finger of a participant on a key to cause an effect (Borhani et al., 2017; Dogge et al., 2012). Temporal binding in these situations is significantly decreased, and effects are much smaller than when the action is voluntary. In short, externally triggered actions seem to 
abolish or even reverse binding, indicating that such behaviors are not accompanied by the sense of agency. Why, then, did our condition in which action timing was externally triggered fail to reduce the sense of agency?

One plausible explanation pertains to the distinction between habits and goaldependent behavior. Habits are conceptualized as responses to external cues that do not depend on the instigation of a goal. Habits are therefore difficult to change as the environment directly triggers them without the mediation of intentional control (e.g., Aarts et al., 1997; Wood \& Rünger, 2016). Research on cognitive neuroscience indicates that a genuine habit is implemented by the sensorimotor striatum loop that corresponds to bottom-up action selection, while goal-dependent behavior is implemented by the cortico-basal ganglia loop that corresponds to deliberation and planning (Yin \& Knowlton, 2006). In doing so, habits represent an instance of no autonomy, and it is plausible that the full absence of intentions in controlling action selection considerably weakens the sense of agency.

However, it is important to note that, in experimental research on human habits, researchers commonly employ task instructions that serve as goals to map stimuli to responses, and to provide context to the task. Therefore, what seems to look like a habit actually is a goal-directed action that is under intentional control (Marien et al., 2019). This may explain why we did not find an effect of no autonomy on intentional binding, because even when participants responded directly to external cues, the action was internally selected as part of the general goal of the task. These findings concur with a study by Wang and colleagues (2017). Combining the Simon-task with an intentional binding task, they instructed participants to press a left or right key to produce a tone. Key presses were triggered by auditory cues that were congruent or incongruent with the spatial location of the cue. Thus, action was more stimulus-based (in congruent trials) and intention-based (in incongruent trials). They found no differences on intentional binding. These findings indicate that while 
action selection and timing were stimulus-based, there was room to represent the sensory effects and to internally select the action to produce them. Finding binding in such a goaldirected behavior context suggests that endowing individuals with 'minimal autonomy', i.e. an agent's ability to internally start up motor processes in response to a cue in order to achieve a goal at hand, is sufficient for agency experiences to emerge (Passingham et al., 2010). This fits well with the compatibilism view on free will and determinism (e.g., Dennett, 2004; Frankfurt, 1971; Gallagher, 2000; Korsgaard, 2009), which emphasizes the opportunity for individuals to live in accordance with one's personal goals and values as the key dimension of autonomy.

The analysis of the role of personal goals and values in the sense of agency may have implications for understanding when personal autonomy undermines the sense of agency. Autonomy might be fully at stake when a person has to act against her own will. As we addressed above, this issue relates to the force of habit, in which a person intends to do A and then the environment triggers action B. However, acting against one's will may also be part of a pervasive social context. In a recent study on coercion, Caspar and colleagues (2016) showed that intentional binding is weakened when participants have to punish another person, suggesting that social pressure can act as a powerful situation to undermine the sense of agency. Milgram (1974) coined this dramatic obedience effect as agentic shift, - the tendency to relinquish personal control to an external agent. Accordingly, whereas forcing people to select and time their action to achieve a personal goal leaves the sense of agency intact, forcing them to act against their goal challenges it. This interplay between autonomy over goals and actions with respect to the sense of agency is somewhat speculative, and more research is needed to further examine how various levels and degrees of autonomy attenuate agentic experiences. Our findings may serve as a starting point for this, offering new insights 
that stimulus-based action selection does not suffice to reduce the sense of agency and hence, to undermine people's belief to be the author of their own behavior. 


\section{Chapter 5}

The Role of Intentional Strength in Shaping the Sense of Agency

Based on: Antusch, S., Aarts, H., \& Custers, R. (2019). The role of intentional strength in shaping the sense of agency. Frontiers in Psychology, 10, 1124. 


\begin{abstract}
Awareness of action is a pervasive personal experience that is crucial in understanding selfgenerated and other-generated actions as well as their effects. A large body of research suggests that action awareness, as measured by the magnitude of temporal binding between an action and its effect in an operant action task (i.e., intentional binding), is rooted in the human capacity to experience self-agency and establish action intentions. Whereas previous research mainly addressed the role of intentionality itself in these socially well-shared experiences, in the present study we focused specifically on one important aspect of it: the quality or strength of action intentions. We expected and established that stronger intentions increase intentional binding. Specifically, the magnitude of the binding effect, as assessed by the Libet clock task in which two actions were followed by the same neutral tone, was elevated for the action that was enacted with stronger intentions. We briefly discuss the implications of the observed role of intentional strength in temporal binding between action and effect, for promoting a better understanding and examination of how the concept of intentionality is associated with action awareness in general, and the experience of being the agent of one's own actions in particular.
\end{abstract}


Intentions constitute an essential building block of human action preparation, action initiation and action awareness. Conceptually, intentions can be defined as mental representations of an individual's upcoming volitional movement. These action representations lie at the basis of our diverse behavioral repertoire and are strongly influenced by learning. Sensorimotor processes become associated with the specific patterns of muscle activity they produce and their observable consequences on a perceptual, sensory, and motor level, allowing actions to be stored in memory together with their consequences. While these associations often operate under the radar of conscious awareness, bringing the action representation of an intention to consciousness increases the probability of preparing and initiating the associated action (Aarts et al., 2008a); as captured by the ideomotor principle (Greenwald, 1970; James, 1890; Shin et al., 2010). According to the ideomotor principle and also more recent theories such as the theory of event coding (TEC), action and effect are bound together by repetition of co-occurrence that eventually are mentally represented in terms of their causal relations (Hommel et al., 2001).

What is more, intentions do not only serve the preparation and initiation of action but also hold an important role in promoting the awareness of action and the experience of selfagency (e.g., Frith et al., 2000). Being aware of one's actions and their consequences in the external world facilitates action planning and is crucial in sharing goals and feelings during social interaction (Baldwin \& Baird, 2001; Frith, 2002). It thus does not come as a surprise that irregularities in intention formation and attribution are associated with disturbed action awareness and problems related to action planning and control (e.g., Blakemore et al., 2002; Voss et al., 2010). These irregularities are also reflected in subjective measurements of sense of agency - the feeling that one is the agent of one's actions and their effects in the external world. Specifically, the sense of agency arises from intentional actions but is diminished for 
unintentional or reflexive actions (Aarts et al., 2008b; Damen et al., 2015; Miller \& Ross, 1975).

Importantly, not only explicit agency reports, but also the implicit nature and manifestation of it are affected by intentionality. In an extension of the original study by Libet and colleagues (1983) - using an adapted version of the Wundt Clock to assess the awareness of single motor movements (e.g., lifting one's finger) -, recent studies have started to examine awareness of action in a context where movements have actual effects in the environment. They reliably find that volitional action (such as a key press) and an external stimulus (such as a tone) that follows the action at a short interval $(250 \mathrm{~ms})$ are shifted towards each other in temporal perception, causing the perceptual compression of the temporal interval between them. Illustrating the significance of volition, this temporal binding effect for operant actions has consistently been shown to be critically occurring for intentional (self-induced) actions and to be absent or weakened for unintentional (externally-evoked) actions, induced passively or by transcranial magnetic stimulation (TMS) (Borhani et al., 2017; Dogge et al., 2012; Haggard et al., 2002). Theoretical explanations predominantly lean on forward prediction or comparator models of motor prediction, centering around the idea of an efferent copy of the motor command being send to an internal prediction model. The incoming external sensory effect of one's action is then being compared with the predicted sensory effect. A sense of agency is believed to arise in the case of a match between the predicted and the actual sensory effect. Similarly, in the case of a mismatch, absence of an agentic experience is expected (Blakemore et al., 2002).

Whereas being the currently most-widely accepted and plausible explanation for the evolvement of (implicit) agency experiences, these models seem to suggest an "all or nothing" mechanism, meaning that solely the absence or presence of an intention is assumed to shape and determine the manifestation of agency. Nevertheless, this construal of 
intentionality does not resemble reality in which individuals might hold stronger or weaker motivations to execute a specific action intention. Hence, whereas the role of intentionality of action in shaping the sense of agency has been unequivocally established, little attention has been given to the quality of these intentions. Aiming to fill this void, in the current paper we focus on this crucial aspect of intentions and investigate whether the sense of agency - as reflected by temporal binding - varies as a function of the (motivational) strength of intentions. The extent of temporal binding, we argue, does not only depend on the intention to action, but also on the strength with which people hold the intention in mind during the task or action context at hand.

In research on the philosophy of mind, intentions are often considered as entities to understand how the brain, mind and body interact in producing observable behaviors in individuals (Bratman, 1999; Dennett, 1993; Searle, 1983). An important suggestion comes from Searle (1983), who made a distinction between prior intention (when an action is planned ahead to reach a goal or outcome) and intention in action (when an action is prepared and initiated in the task at hand) that are independently motivated. Whereas both types of intentions are important for actions to occur, intention in action is crucial for the emergence of action awareness. Following an empirical approach to predict the occurrence of intentional actions, theories in psychological science put forward the idea that intentions to engage in an action vary in strength, based on the expected value or importance of performing the action (e.g., Aarts et al., 2012; Ajzen \& Fishbein, 1977; Sheeran, 2002). Actions that are expected to be of higher subjective value (such as actions that are rewarded for their execution) are thought to accommodate stronger intentions than actions that are of lower subjective value (Eitam \& Higgins, 2010). Stronger intentions have been shown to be better remembered, are more readily implemented, produce more effort in the case of obstacles, and are more 
sustainable (Gollwitzer, 1993; Gollwitzer, 1999; Walter \& Meier, 2014). In short, action intentions that carry more motivational strength are more likely to be enacted.

Recently, research has started to explore the motivational underpinnings of intentions and their role in shaping the sense of agency. Several studies have indicated that motivational manipulations such as variations in the value of action outcomes can affect temporal binding, although the direction of these effects is not always clear. Some studies found that binding increased when an individual's intentional action caused financial gains as compared to financial losses (Takahata et al., 2012) or is followed by positive (versus negative) emotional outcomes (Yoshie \& Haggard, 2013; but see: Moreton et al., 2017; Ruys \& Aarts, 2012; for no differences in effects of emotional valence). Contrary though, intentional binding was shown to also increase for severely as compared to moderately negative (moral) outcomes (Moretto et al., 2011). Investigating the sense of agency in a coercion setting, Caspar and colleagues (2016), on the other hand, demonstrated that intentional binding was unaffected by the outcome valence when individuals intentionally inflicted financial or physical pain on another participant and were not forced to do so (Caspar et al., 2016).

Whereas suggestive, these findings do not directly speak to the role of intentional strength in action. They suggest an influence of value or importance variations of the actionoutcomes on the implicit sense of agency, but they do not isolate the role of intentional strength in action from the outcome of action: participants in these studies are instructed to focus on different outcomes of actions, rather than the action itself, which confounds intention in actions with prior intentions. Put differently, the differences in binding strength could either be explained in terms of retrospective cognitive influences based on the value and type of outcomes or by an increase in strength of intention to execute the action. Hence, to test the role of strength of action intention in intentional binding, one should not vary the value and type of outcomes, but keep the outcome neutral and constant. 
Circumstantial evidence for the role of intentional strength in the sense of agency was obtained by Aarts et al., (2012), who examined the effect of incidental processing of positive (vs. neutral) affect on executing action intentions in an operant action task on intentional binding. In their study, participants were briefly exposed to neutral or positive pictures before their preparation and enactment of the intention to press the spacebar on a keyboard which resulted in the presentation of one single tone. They capitalized on the notion that positive stimuli serve as a rewarding motivational drive, increasing striatal dopamine functioning. Striatal dopamine facilitates sensorimotor processes that play a role in the actual initiation of an action, the processing of outcome feedback and the experience of operant action (Aarts et al., 2012; Aston-Jones \& Cohen, 2005; Dreisbach \& Goschke, 2004). In line with this notion, they established that positive affect enhanced the temporal binding between the intended key press and the resulting tone. It should be noted, though, that the positive affective stimuli were irrelevant for the action intention, and hence, it remains unclear whether the indirect effect on intentional binding is the result of increased intentional strength or due to induced positive affect.

In the current research, we aimed to more directly test the influence of the motivational aspect relating to intentional strength on action awareness by addressing its influence on temporal binding. In doing so, we manipulated intentional strength directly in a task where two actions were differently rewarding, while both resulted in a neutral and fixed single outcome. Using monetary rewards to manipulate intentional strength, we induced a motivational preference for one action over another, otherwise identical, action. In comparison to other studies, we thus did not compare positive or negative action outcomes, such as gains and losses, but orthogonally manipulated the motivation of the actions itself by manipulating their expected value. In a counterbalanced within-subjects design, participants learned that a key press was more frequently rewarding (80 percent reward probability) than 
an alternative key press ( 20 percent reward probability). That is, any effects would not be due to differences in the direction of the valence of the outcome of the actions (e.g., negative or positive) but solely due to strengthened intentions to execute a specific action based on their expected rewarding quality (left versus right key press). Following, we hypothesized the more rewarding action to carry more intentional strength and thereby increasing intentional binding of the action and the neutral effect.

\section{Methods}

\section{Participants and Design}

Thirty-six participants ${ }^{12}(M=21.64, S D=4.42$ years $)$ with normal or corrected to normal vision took part in the experiment in exchange for monetary reimbursement. The experiment employed a 2 (target of judgment: action vs. effect) x 2 (type of trial: baseline vs. operant) x 2 (reward frequency of key: low reward frequency vs. high reward frequency) within-subjects design.

The experiment was carried out in accordance with the guidelines of the declaration of Helsinki and approved by the ethical committee of the Faculty of Social and Behavioral Sciences, Utrecht University, as part of an overarching ethical application covering a project line using the Libet clock task (ethics approval code FETC17-124). All participants gave written informed consent.

\section{Procedure}

Participants were sat in separate cubicles, approximately $60 \mathrm{~cm}$ away from a computer screen. The experiment was programed using Eprime 2.0 and all instructions were provided

\footnotetext{
${ }^{12}$ The sample size was therefore based on similar study in our lab, $\mathrm{n}=28$ (Aarts et al., 2012). Since most previous studies testing moderator effects on intentional binding used smaller samples, we aimed to ascertain that we test a sufficiently large sample.
} 
on screen. To assure adherence to the instructions, the experimenter was present during the whole duration of the experiment.

Participants completed three different experimental stages in sequential order: a preference induction phase, a tone acquisition phase and four randomized blocks of intentional binding trials.

Preference induction. The experiment utilized two different actions, pertaining to key presses ('x' vs. 'n') on a QWERTY keyboard. Key presses had different statistical probabilities to yield a monetary reward of five Eurocent. For each participant, one of the keys ('x' vs. 'n') was of 'low reward frequency' ( 20 percent reward probability) whereas the other was of 'high reward frequency' ( 80 percent reward probability), equaling average expected rewards of one and four Eurocent respectively. Participants were explicitly informed about these fixed probabilities. Key - reward probability mappings were counterbalanced across participants.

Before the beginning of the actual experiment, participants completed 40 induction trials. Each trial began with the Dutch equivalent of the statement "The following key press is worth 5 [0]) Eurocent" presented in the middle of the screen for $3000 \mathrm{~ms}$, followed by a command to press either the ' $\mathrm{x}$ ' (left) or ' $\mathrm{n}$ ' (right) key on the keyboard. This command stayed on screen until participants had pressed the correct key. Key presses were equally distributed across the total amount of induction trials (i.e., 20 trials per key). The aim of the induction trials was to induce a distinct preference for the high reward frequency key over the low rewarding key in participants.

Tone acquisition phase. Subsequently, participants learned that both key presses have the same causal effect - a neutral $1000 \mathrm{~Hz}$ sinus tone of $100 \mathrm{~ms}$ length. To avoid confounding 
intentional strength with effect identity, it was emphasized that the tone (identity) would not signal reward obtainment, meaning that the reward was solely linked to the action executed.

In total, participants completed ten practice trials (five trials per key press). Each trial began with a command to press a key which remained on screen until participants pressed the correct key. Participants' key press was then followed by the $1000 \mathrm{~Hz}$ sinus tone $250 \mathrm{~ms}$ later. Because the purpose of these trials was only to acquaint participants with the effect of their key presses, they were not rewarded.

Intentional binding trials. Following, the actual experiment started. In total, participants completed four randomized intentional binding blocks - two baseline blocks (baseline action, baseline effect) and two operant blocks (operant action, operant effect). Each block consisted of 40 experimental trials and five practice trials which were not included in the analysis, resulting in a total of 180 trials. Trials in which participants had to press a key (baseline action, operant action, operant effect), began with a command indicating which key to press, presented in the center of the screen for $2000 \mathrm{~ms}$. Both key presses were distributed equally across trials, resulting in 20 trials per key. The order of trials within a block was randomized.

Next, the clock face appeared on the screen. Trials used an adapted version of the Libet clock method (Libet et al., 1983). Participants attended to a dotted clock face, composed of 40 grey dots arranged in a circle with a diameter of six $\mathrm{cm}$ from the center of the screen. A black dot that moved at a period of $2560 \mathrm{~ms}$ per rotation served as the clock hand. The clock hand started moving from a random position on the clock face. To assure attentional focus, participants were instructed to wait with pressing the key until the clock hand had completed one full rotation and vary the moment of their key presses across trials. In operant trials, the key press was followed by the $1000 \mathrm{~Hz}$ sinus tone $250 \mathrm{~ms}$ later. In baseline action trials, the key press was not followed by the tone. In baseline effect trials, participants did not execute 
an action but the tone was played at a varying moment between $2560 \mathrm{~ms}$ and $5120 \mathrm{~ms}$ after the initial presentation of the clock face.

Before disappearing, the clock hand rotated further for $1000 \mathrm{~ms}$ to avoid that participants would simply report the last remembered position of the clock hand. After the disappearance of the clock hand, a prompt to judge the temporal onset of the target (i.e. key press or tone) using the mouse cursor appeared in the middle of the clock face. After a time judgment had been made, the clock face disappeared and reward feedback was presented in the middle of the screen. Reward frequencies were the same as in the preference induction phase (20 vs. 80 percent for the low and high reward frequency key respectively). In effect baseline trials, rewards were randomly distributed across trials, resulting in 50 percent of the trials being rewarded and the same averaged expected reward value as in other blocks. The inter-trial interval was set to $1000 \mathrm{~ms}$.

At the end of experiment, participants filled in several questions pertaining to their demographics such as age, handedness and gender as well as their overall motivation. Finally, they were thanked for their participation and reimbursed.

\section{Results}

\section{Data set and variables}

Following Aarts et al., (2012), time judgements that exceeded or preceded the actual value of the clock hand by $640 \mathrm{~ms}$ (i.e., 10 dots) were excluded from analysis as they were likely to be due to inattentiveness of the participant. Less than one percent $(0.31 \%)$ of the trials was excluded based on this criterium.

\section{Results}


Separate mean judgment errors in milliseconds were calculated for the different cells of the design and subjected to a repeated measures ANOVA with target, type of trial and reward frequency of the key as within-subjects factors. Results revealed a significant main effect for target, $F(1,35)=4.63, p=.04, \eta p^{2}=.12$. That is, the temporal perception of key presses $(M=17.28, S D=49.92)$ was delayed whereas tones $(M=-7.23, S D=57.98)$ were anticipated. Moreover, the intentional binding effect was replicated, $F(1,35)=40.19, p<$ $.001, \eta \mathrm{p}^{2}=.54$. Temporal perception of key presses was later in operant trials $(M=39.62, S D$ $=70.27)$ than in trials in which the key press was not followed by the tone $(M=-5.06, S D=$ 43.78), $p<.001$. Similarly, tones that were initiated by a key press were perceived to occur earlier in time $(M=-33.86, S D=78.34)$ than tones that did not follow an action $(M=19.46$, $S D=58.37), p<.001$

This was further qualified by a three-way interaction between target, type of trial and reward frequency of the key press, $F(1,35)=6.17, p=.02, \eta p^{2}=.15$ (see Figure 1). Simple main effects analysis revealed that the effect was driven by tone binding. That is, the temporal perception of the action in both, baseline and operant trials, was not altered when a low or a high reward frequency key was pressed. Interestingly though, tones that were caused by an action were perceived earlier when the key was more frequently rewarding $(M=-38.02, S D=$ 81.27) as compared to when the key was less frequently rewarding $(M=-29.76, S D=76.4), p$ $=.02$. That is, intentional binding was stronger for trials in which participants executed an action that was more frequently rewarding as compared to trials in which the action was less frequently rewarding. 


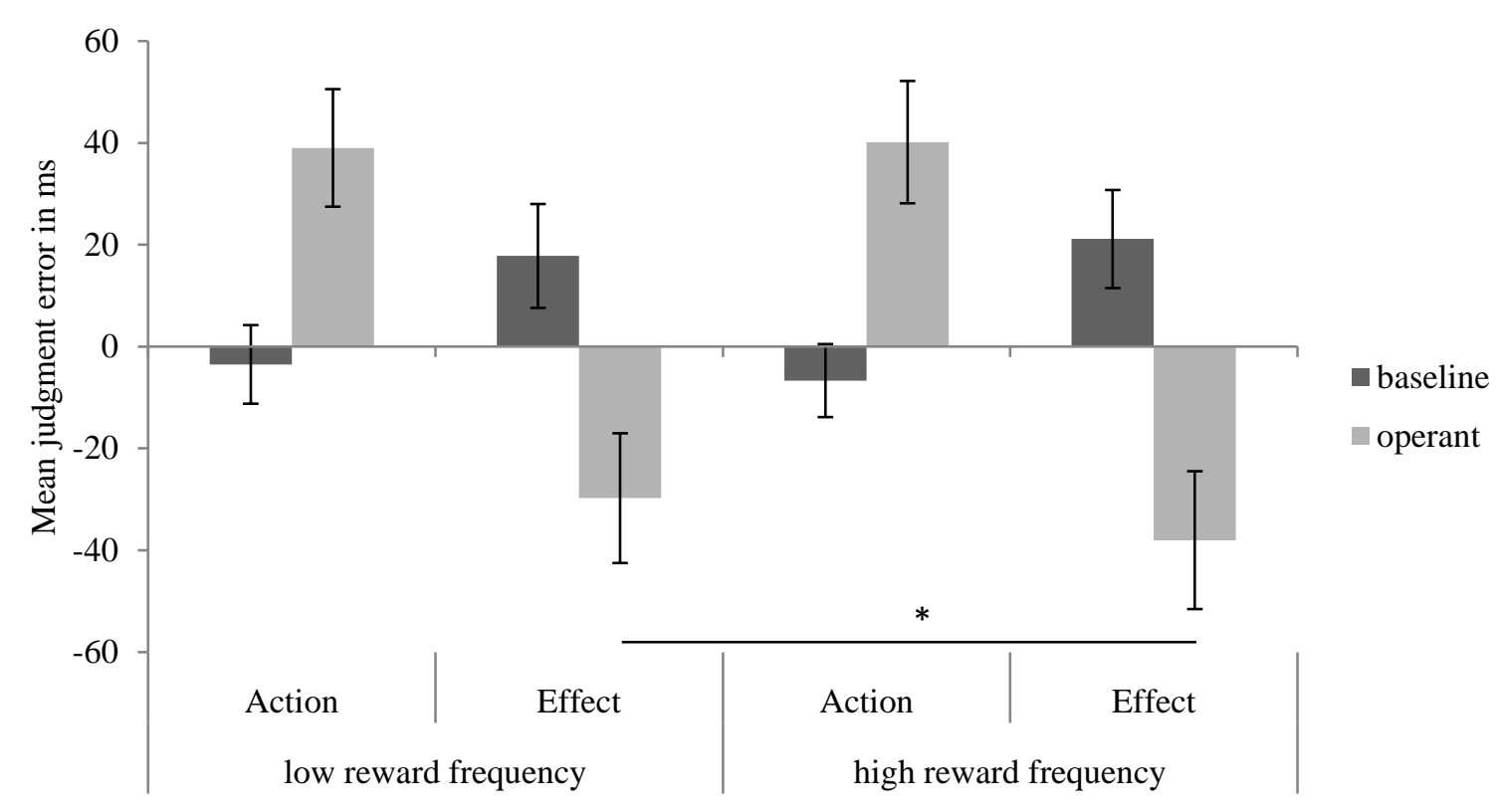

Figure 1. Mean judgment errors in ms for action and effect across type of trial and reward frequency of the key. Bars represent standard errors of the mean. * $p=.02$.

\section{Discussion}

The general importance of intentionality for the emergence of action awareness and agentic experiences has been widely established, with sense of agency being diminished when intentionality is absent. Interestingly though, little research has examined the influence of the quality of these intentions - or intentional strength - on (implicit) agency. Aiming to fill this void, in the current research we investigated how the implicit experience of self-agency changes when the operant action is enacted by stronger versus weaker intentions based on the expected value of the action. Confirming our hypothesis, temporal binding indeed increased for trials on which participants pressed a frequently rewarded key, suggesting a magnified implicit sense of agency. More specifically, the temporal onset of the outcome was significantly anticipated whereas the perception of the temporal onset of the action was not significantly altered. Thus, we were able to demonstrate that not only the presence of 
intentions, but also their motivational value alters the implicit processes of the sense of agency.

Our findings are in line with theoretical frameworks emphasizing the importance of motivational relevance in intention formation, such as the relevance of a representation framework (ROAR), which suggests that action representations carrying a stronger motivational value are more actively held in mind and thus also more likely to influence behavior and experiences (Eitam \& Higgins, 2010). Generally, subjectively more relevant stimuli evoke brain activity significantly earlier than subjectively less relevant stimuli (e.g., Schultz, 2006; Schupp et al., 2004), which facilitates intention formation and action execution. In our experiment, the action cue for the high reward frequency key would be such a motivationally relevant stimulus, activating a strong action representation and giving rise to an enhanced efference copy. This, in return, would cause a more pronounced intentional binding effect. On a neurobiological level, this can be explained in terms of a dopaminergic boost. The action cue at the beginning of a trial carries reward-related information which causes dopaminergic activation in the ventral striatum. This dopaminergic activation facilitates the transferal of information from the pre-frontal cortex to cortical motor areas, affecting voluntary action as well as the awareness thereof (Aarts et al., 2012; Schultz, 2006).

Although speculative, such an explanation in terms of increased action preparation would also be in line with earlier research on inter-individual differences in the experience of intentionality and free will beliefs in agency contexts. Specifically, past research showed correlations between intentional awareness and brain activation related to motor preparation for individuals who are assumed to enjoy high levels of internal insight (e.g., meditators; Jo et al., 2015), as well as demonstrated that these differences can affect the implicit sense of agency. A study by Lush and colleagues (2016), for example, found that intentional binding was stronger for experienced meditators who find practicing mindfulness pleasurable, than for 
controls (Lush et al., 2016). In addition, beliefs in free will have been linked to intentional binding, which is explained in terms of a motivational or cognitive orientation towards the results of action intentions (Aarts \& van den Bos, 2011; Lynn et al., 2014). Together, these findings suggest that stronger experiences of intentions have a special status in motivating people towards preparing actions and tuning them for predicting or anticipating feedback information that will result from enacting intentions. On this view, intentional strength increases the sense of agency by motivational (action preparation) as well as cognitive (effect anticipation) processes.

There is previous research that concurs with the findings reported in the present paper. Specifically, motivation manipulations have been used to increase the value of outcomes of actions, and to test the role of such motivational enhancement on intentional binding. The general gist of these studies is that outcome importance modulates binding between action and outcome under free (voluntary) as well as coercive (involuntary) conditions of behavior (Caspar et al., 2016; Moreton et al., 2017; Takahata et al., 2012; Yoshie \& Haggard, 2013). While these finding are supportive of the role of intentional strength, it is not clear whether they are due to motivational enhancement of action intentions or prior intentions. Teasing these two different accounts apart is important: They might shed light on the question whether action awareness is a direct function of the strength of intention in action and the subsequent preparation and initiation of it (Libet, 1985), or relies on a prior intention that includes information about the importance of outcomes of action. As has been argued and shown, prior intentions increase attention to, and strengthen anticipation of desired outcomes (Aarts, 2012), and readily evoke a retrospective inference process after action execution that produces postconscious thoughts about agency and behavior (Aarts et al., 2005; Wegner, 2003). On this view, prior intentions effects on intentional binding are not the result of motivational 
processes per se, but could rather be considered as cognitive effects in which humans use causal knowledge about their action and potential effects to inform themselves about agency.

Whereas our findings favor an intentional strength account for action, we would like to address a few issues that limit the interpretation of the results of our study. The first issue relates to the absence of free choice in action selection. We wanted to ensure that intentional strength rather than preferences would modulate the intentional binding effect. Since preferences would however always confound free choice, we controlled for action preferences by instructing participants which key to press on a given trial. Nonetheless, it could be argued that this absence of free choice in action selection does not allow for, or at least negatively impacts, intention formation. Research by Barlas and Obhi (2013), for example, showed decreased intentional binding when participants had limited action alternatives (Barlas \& Obhi, 2013). Nevertheless, binding, albeit decreased, did not disappear for conditions in which individuals were given moderate action selection options or no choice. Furthermore, it is important to note that the original intentional binding study (Haggard et al., 2002b) established a strong and replicable intentional binding effect, even though only one key could be pressed, and no other choice alternatives that causally linked the action to the effect existed (Haggard et al., 2002b). This suggests that for intentional binding to arise, it suffices to hold an intended action in mind and to choose the moment to act on this intention (Brass and Haggard, 2008). Thus, while action intentions were externally provided by instructions rather than self-generated, participants could choose to time their action that, in principle, represents the intention in action upon one's own will (Searle, 1983).

Another related issue that needs to be addressed concerns the question of whether externally induced action intentions can be viewed as being rooted in the intentionality of action at all. To answer this question, one needs to assume that external (instructed) and internal (self-chosen) intentions differ in their effects on behaviour. Whereas self-generated 
and externally generated intentions might differ in their phenomenological experiences, research suggests that both type of intentions can have similar effects on behaviour. Research on goal-setting theory, for example, shows that self-assigned and other-assigned goals do not necessarily result in performance differences, especially when both types of goals are important (Locke \& Latham, 1994; 2019). Furthermore, there is recent empirical evidence that externally induced action intentions can produce similar effects on intentional binding as self-generated action intentions (Wang et al., 2017). In an attempt to examine intentional binding effects in a Simon task, Wang and colleagues (2017) asked participants to respond immediately to a cue, causing a tone to occur. Thus, whereas knowledge about the causal relation between action and effect was present, both choice and timing of the action were fully pre-determined. Their findings demonstrated that even under such strong externally forced action intentions, intentional binding occurred to a similar degree as found for self-timed intention effects.

These findings raise the question of whether intentions should only be defined in terms of what, when and whether at all, or whether other factors might be more important and decisive in binding action and effect together and creating a sense of agency, such as causal beliefs and other knowledge-based predictions about action and effects (Dogge et al., 2012; van der Weiden et al., 2011). Indeed, it has been repeatedly argued that such beliefs and knowledge play a pivotal role in the way people form intentions and consider themselves as active agents that can engage in action performance to realize their intentions and goals (e.g., Ajzen, 1991; Bandura, 1977). Thus, whereas rewards might create stronger intentions for future actions during learning, the enhanced intentional strength itself might render causal beliefs more salient, supporting people to more strongly predict the effects of their actions, leading to a stronger sense of agency. 
To conclude, we observed that actions that are more rewarding led to a stronger sense of agency in an intentional binding task. These effects could not be attributed to preferences for actions or their effects. The mere fact that stronger action intentions led to stronger intentional binding suggests that intentional strength is associated with action awareness: individuals might become more readily aware of actions that are furnished with strong intentions. We do not know yet whether this intention-action-awareness relationship results from motivational or cognitive mechanisms. However, we hope and believe that the concept of intentional strength might offer an important addition to the study of intentional binding in general, and more specifically to the understanding of how intentionality is represented in the conscious awareness that people have in experiencing themselves as the agent of their own behavior. 


\section{Supplementary Materials of Chapter 2}

JASP Output of the Robustness Check concerning the Results of the Bayesian Analyses

Figure 2.1. Robustness check Bayesian t-test on overall repulsion in Experiment 1.

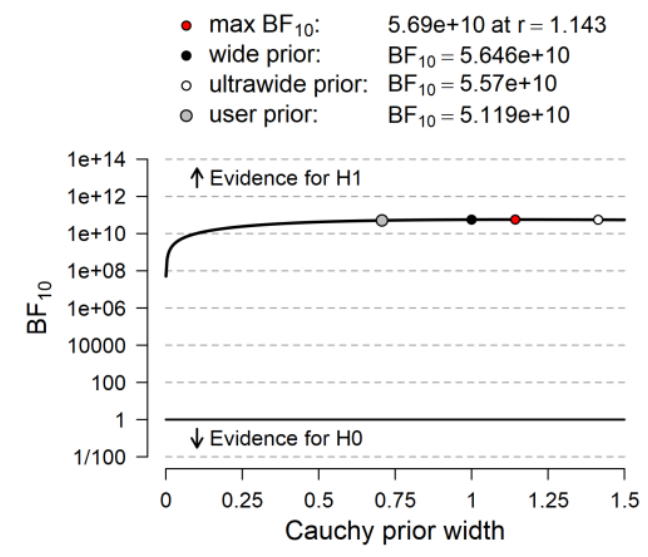

Figure 2.2. Robustness check Bayesian t-test on temporal repulsion between the conditions in Experiment 1.

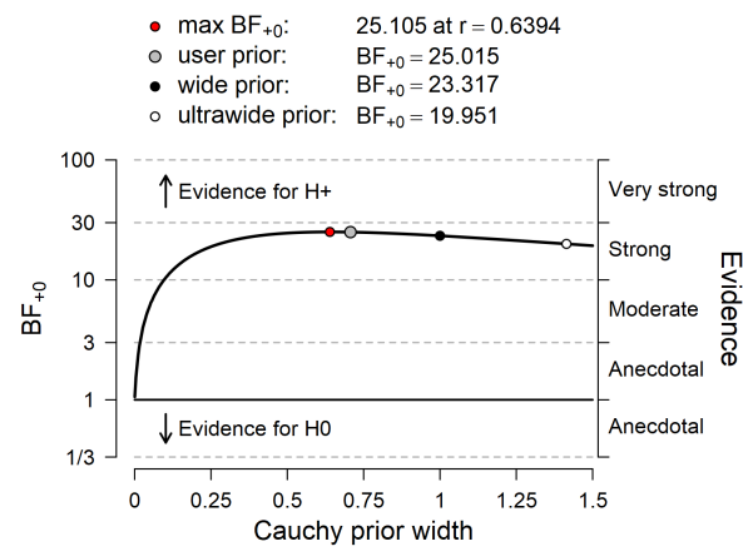


Figure 2.3. Robustness check Bayesian t-test on temporal repulsion in the control condition of Experiment 1.

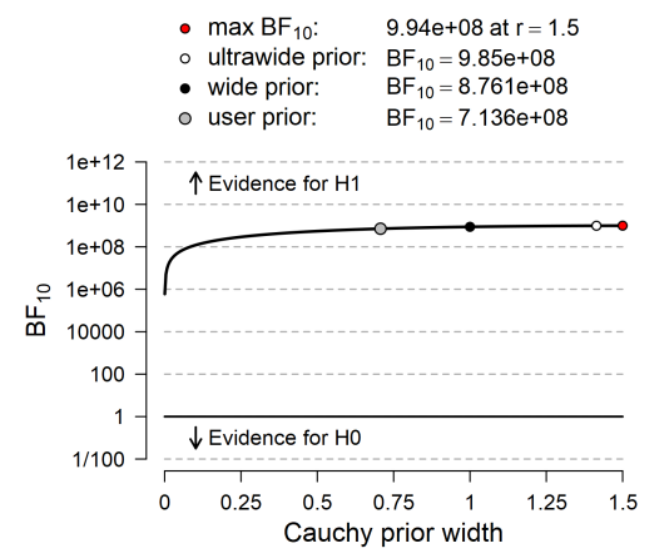

Figure 2.4. Robustness check Bayesian t-test on temporal repulsion in the causal belief condition of Experiment 1.

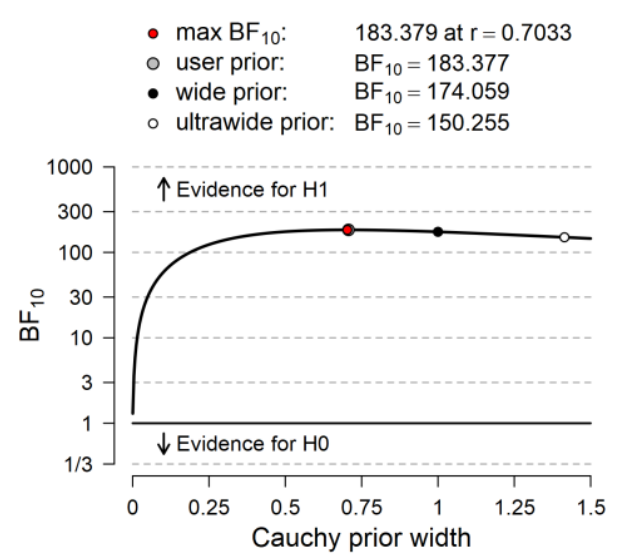


Figure 2.5. Robustness check Bayesian t-test on overall repulsion in Experiment 2.

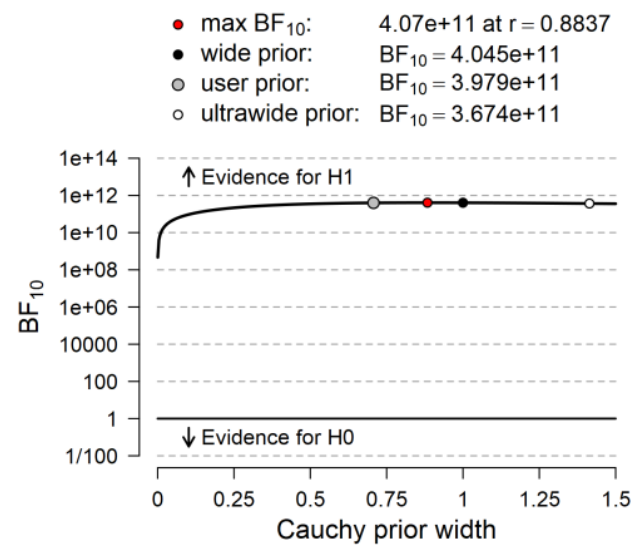

Figure 2.6. Robustness check Bayesian t-test on the difference between the control and causal belief condition in Experiment 2.

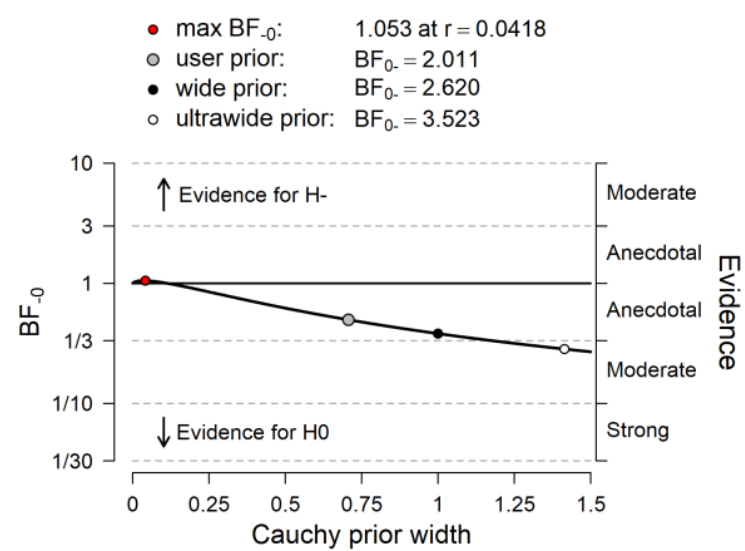


Figure 2.7. Robustness check Bayesian t-test on temporal repulsion in the control condition of Experiment 2.

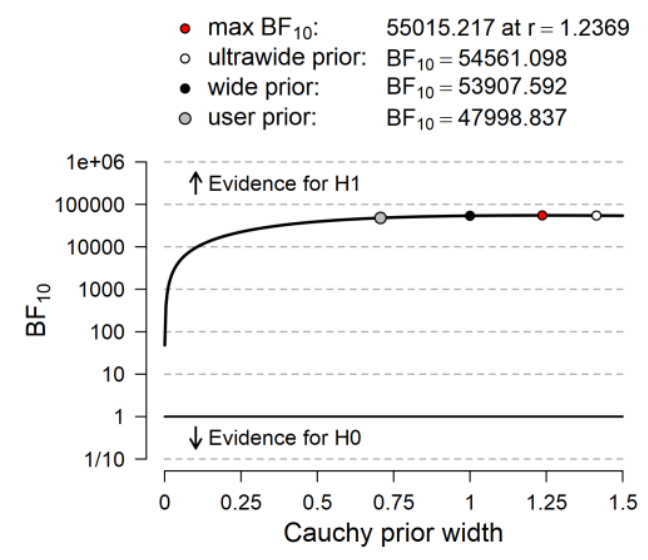

Figure 2.8. Robustness check Bayesian t-test on temporal repulsion in the causal belief condition of Experiment 2.

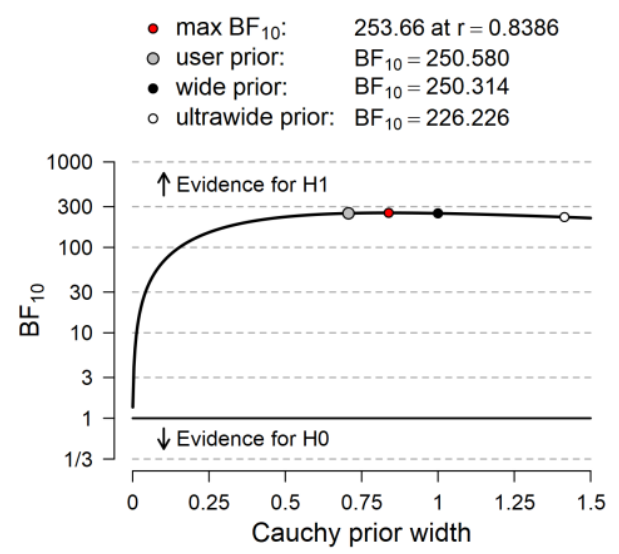


Figure 2.9. Robustness check Bayesian t-test on temporal repulsion in the control + learning condition of Experiment 2.

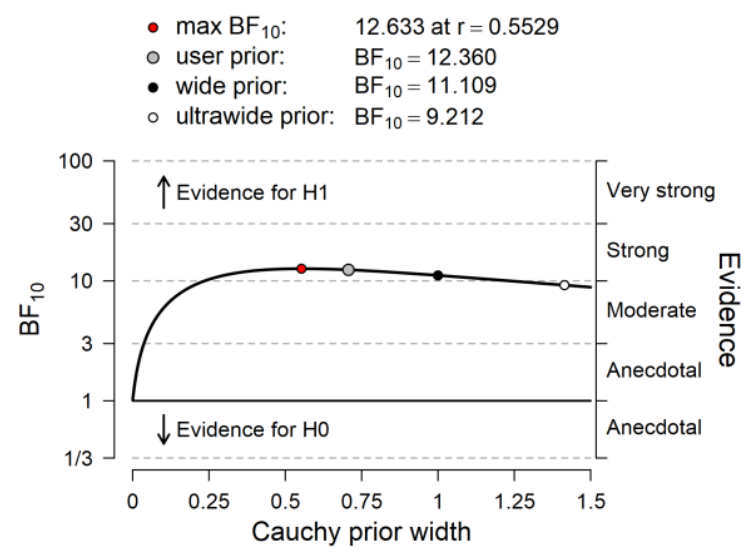

Figure 2.10. Robustness check Bayesian t-test on temporal repulsion in the causal belief + learning condition of Experiment 2.

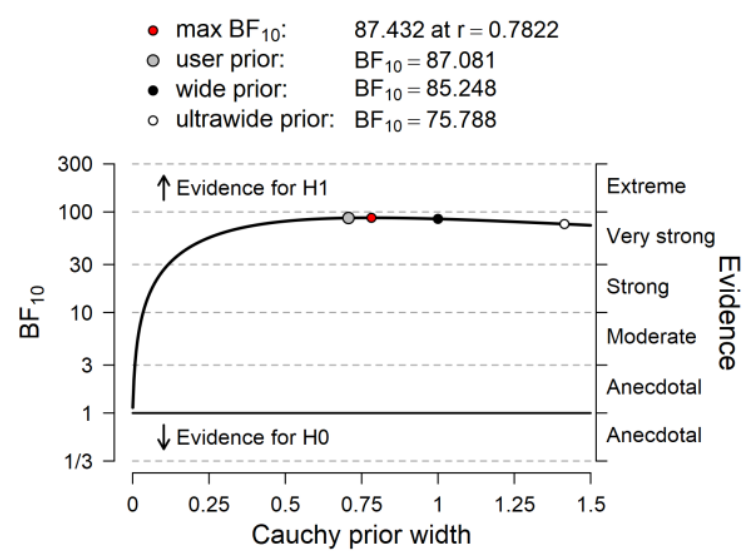




\section{Supplementary Materials of Chapter 3}

\section{Power Calculations}

Experiment 1: As to the best of our knowledge, no literature testing a comparable effect in a tactile-auditory stimulus-based paradigm was available at the point of data collection. Hence, we decided to treat each experiment as a new data point and calculated the sample size on the basis of a moderate effect. We used G*Power 3.1 (Faul et al., 2007) with the following parameters: $\mathrm{f}=.25, \alpha=.05$, power $=.80$, nonsphericity correction $\varepsilon=1$ and $\mathrm{r}=$ .5 to determine the sample size in an ANOVA: Repeated measures within (2) factors. This yielded an estimated sample size of 24 participants. Based on lab capacities, we sampled three extra participants.

Experiment 2: Experiment 1 yielded a repulsion effect that was fairly strong. However, we had no hypothesis about the cue effect size. Therefore, for Experiment 2 we again calculated the sample size according to the following parameters: $\mathrm{f}=.25, \alpha=.05$, power $=.80$, nonsphericity correction $\varepsilon=1$ and $r=.5$ to determine the sample size in an ANOVA: Repeated measures within (3) factors, yielding a sample size of 16 participants. To avoid power issues, we opted for a more conservative (doubled) sample size of at least $n=32$.

Experiment 3: The sample size of Experiment 3 was estimated using the following parameters: $\mathrm{f}=.25, \alpha=.05$, power $=.80$, nonsphericity correction $\varepsilon=1$ and $r=.5$ to determine the sample size in an ANOVA: Repeated measures within (3) factors yielded an estimated sample size of 16 participants. We again doubled the sample size and aimed to recruit a minimum of 32 participants. 
Citation: Faul, F., Erdfelder, E., Lang, A.-G., \& Buchner, A. (2007). G*Power 3: A flexible statistical power analysis program for the social, behavioral, and biomedical sciences.

Behavior Research Methods, 39, 175-191.

\section{Data Preparation and Calculation of Dependent Variables}

For each participant, mean judgment errors per block and separate shifts for the tactile and auditory stimulus were computed. Extreme judgment errors more than $+/-640 \mathrm{~ms}$ away from the actual temporal onset were excluded as inattentiveness on those trials can be assumed (Aarts et al., 2012). This resulted in the exclusion of .19\% of all trials in Experiment 1,.7\% of all trials in Experiment 2 and $.42 \%$ of all trials in Experiment 3.

Perceptual shifts were calculated by subtracting the mean judgment error of baseline trials from the mean judgment error of succession trials (tactile shift $=$ mean judgment error of succession tactile trials - mean judgment error of baseline tactile trials; tone shift $=$ mean judgment error of succession tone trials - mean judgment error of baseline tone trials). Finally, overall binding scores were computed by subtracting the shift of the second stimulus from the shift of the first stimulus (i.e., overall score $=$ tactile shift - tone shift). Positive overall binding scores thus indicated temporal compression of the interval between the tactile and auditory stimuli (temporal binding) while negative scores were indicative of temporal repulsion.

In Experiment 2 and 3, next to overall binding scores, we also calculated two separate binding scores for the two conditions.

Citation: Aarts, H., Bijleveld, E., Custers, R., Dogge, M., Deelder, M., Schutter, D., \& van Haren, N. E. (2012). Positive priming and intentional binding: Eye-blink rate predicts reward information effects on the sense of agency. Social Neuroscience, 7(1), 105-112. 


\section{Bayesian Analysis}

Figure 3.1. Robustness check Experiment 1.

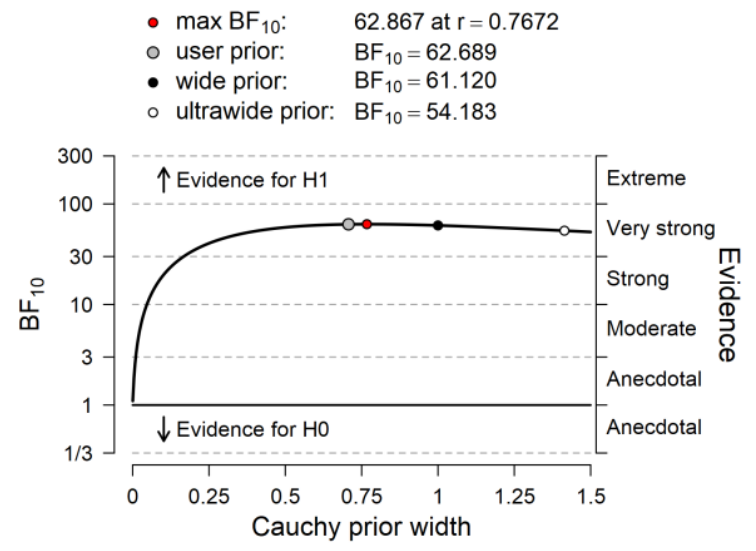

Figure 3.2. Robustness check Experiment 2 testing overall binding against zero.

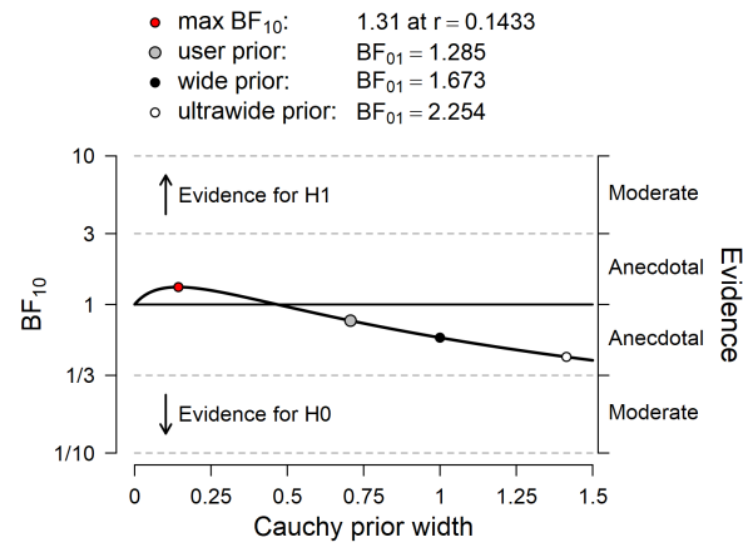


Figure 3.3. Robustness check Experiment 2 testing the difference between conditions.

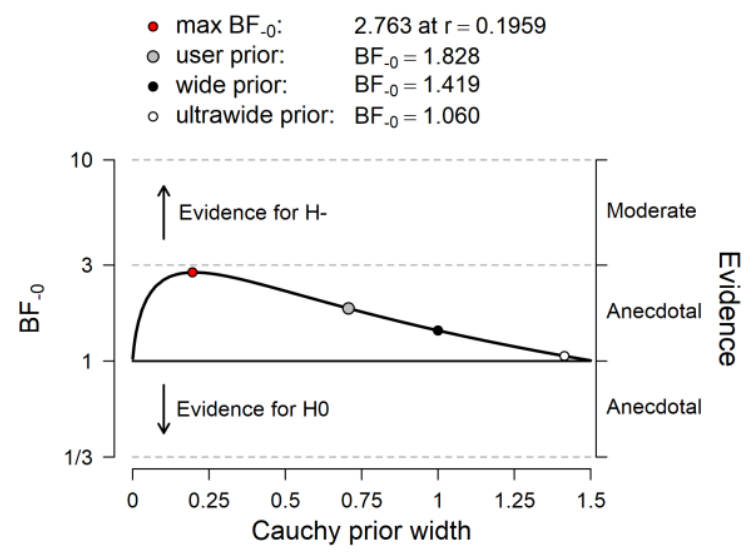

Figure 3.4. Robustness check Experiment 2 testing temporal binding score against zero (no cue control condition).

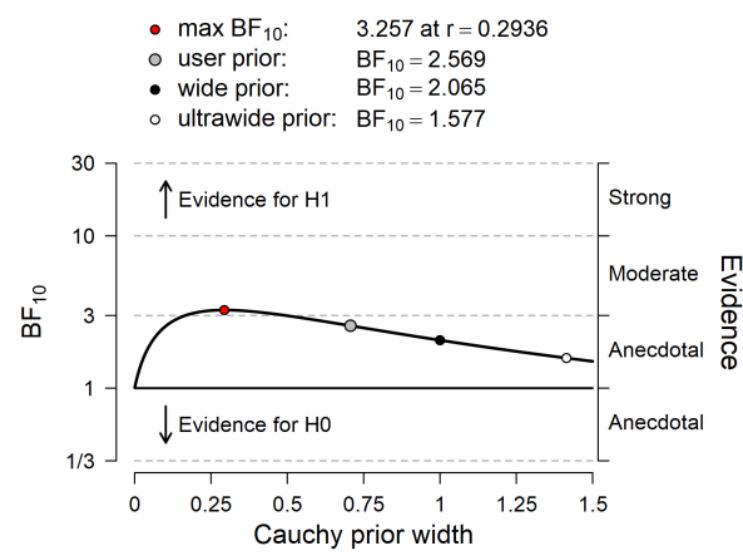


Figure 3.5. Robustness check Experiment 2 testing temporal binding score against zero (cue condition).

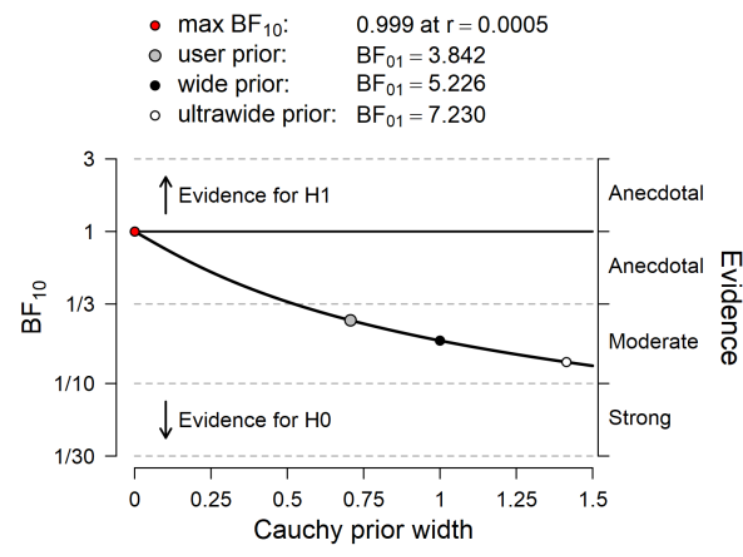

Figure 3.6. Robustness check Experiment 3 testing overall binding against zero.

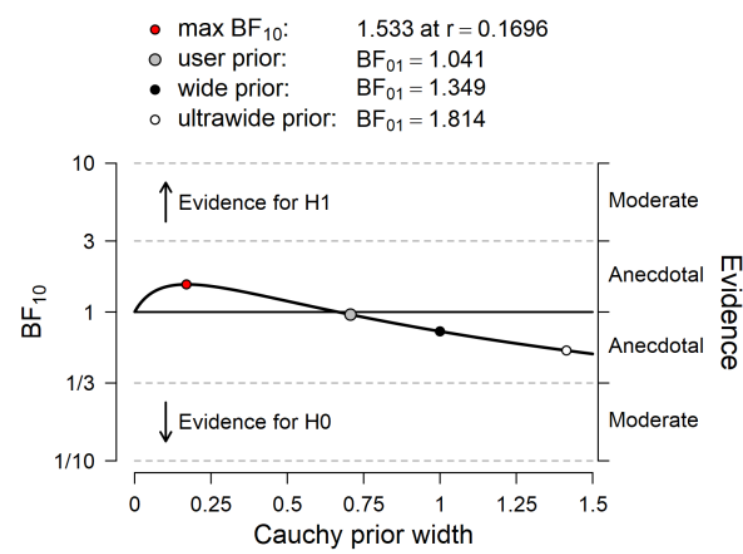


Figure 3.7. Robustness check Experiment 3 testing the difference between conditions.

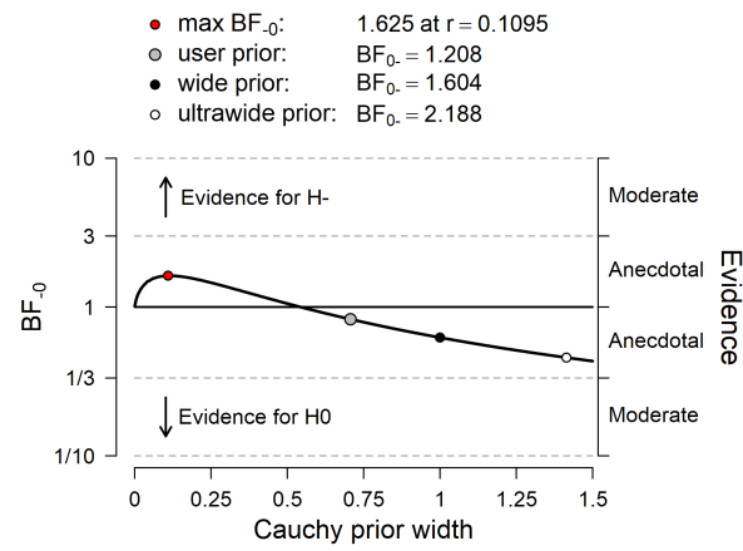

Figure 3.8. Robustness check Experiment 3 testing temporal binding score against zero (direction cue condition).

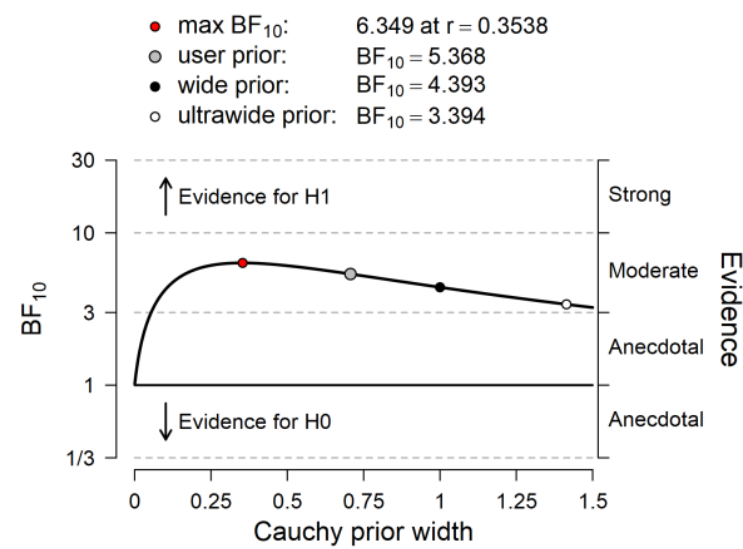


Figure 3.9. Robustness check Experiment 3 testing temporal binding score against zero (direction + countdown cue condition).

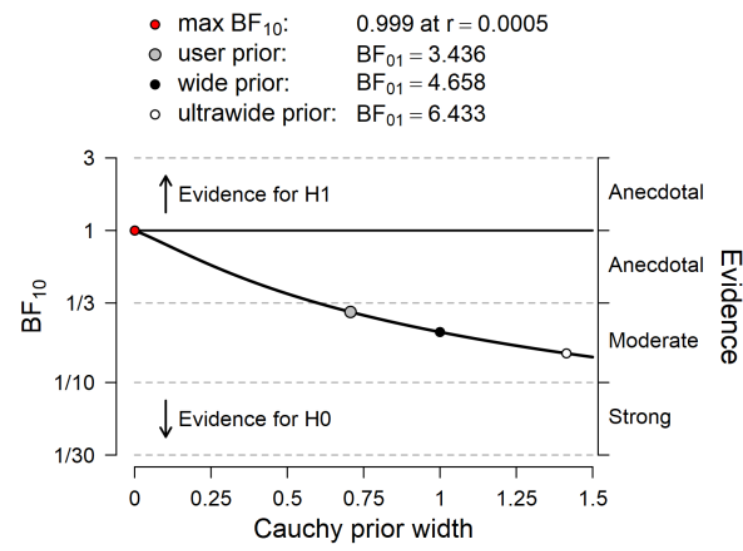




\section{Supplementary Materials of Chapter 4}

\section{Power Calculations}

Experiment 1: The sample size was determined based on an a-priori power analysis conducted in G*Power (Faul et al., 2007). The sample size was determined for a one-tailed ttest with a medium effect-size equal to $d=.5$, an alpha level of .05 and a power of .80 . The calculation yielded an estimated necessary sample size of 27 participants. Based on lab capacities and to offset possible exclusions, we ran two additional participants.

Experiment 2: An a-priori power analysis using $\mathrm{G}^{*}$ Power (Faul et al., 2007) was conducted to estimate the necessary sample size. Since there was no literature using a similar design at the point of data collection, we based our sample size on a power calculation with the following parameters: ANOVA repeated measures design (within factors),effect size $\mathrm{f}=$ .25 , alpha level equal to .05 , power $=.08$, number of groups 1 , number of measurements equal to $2, r=.5$ and nonsphericity correction epsilon equal to 1 . The calculations yielded an estimated sample equal to 34 . Based on lab capacities and to offset possible exclusions, we collected an additional eight participants. Due to technical problems, the data of three participants was lost, resulting in a final sample of 39 participants.

Experiment 3: An a-priori power analysis using G*Power (Faul et al., 2007) was conducted to estimate the necessary sample size. Since there was no literature using a similar design at the point of data collection, we based our sample size on a power calculation with the following parameters: ANOVA repeated measures design (within factors), effect size $\mathrm{f}=$ .25 , alpha level equal to .05 , power $=.08$, number of groups 1 , number of measurements equal to $2, r=.5$ and nonsphericity correction epsilon equal to 1 . The calculations yielded an estimated sample equal to 34. Based on lab capacities and to offset possible exclusions, we collected an additional seven participants. 
Citation: Faul, F., Erdfelder, E., Lang, A.-G., \& Buchner, A. (2007). G*Power 3: A flexible statistical power analysis program for the social, behavioral, and biomedical sciences. Behavior Research Methods, 39, 175-191. 


\section{JASP Output Robustness Check for the Bayesian Analysis}

Figure 4.1. Robustness check Experiment 2 testing differences in temporal binding between conditions

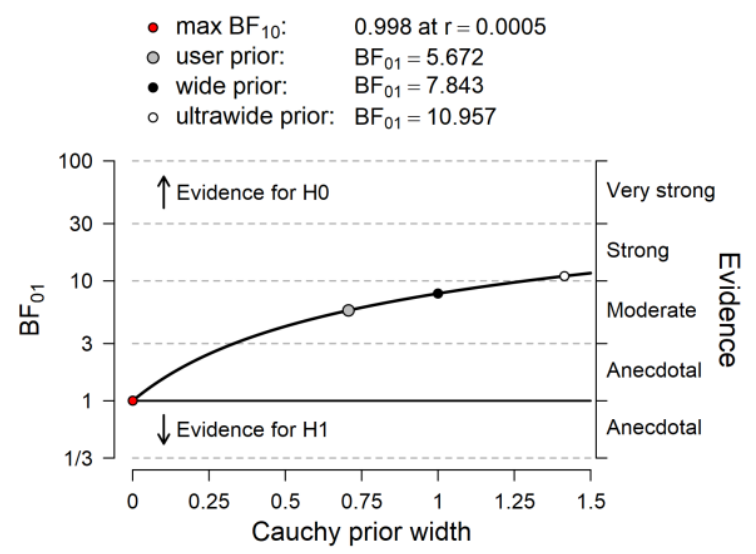

Figure 4.2. Robustness check Experiment 3 testing differences in temporal binding between conditions

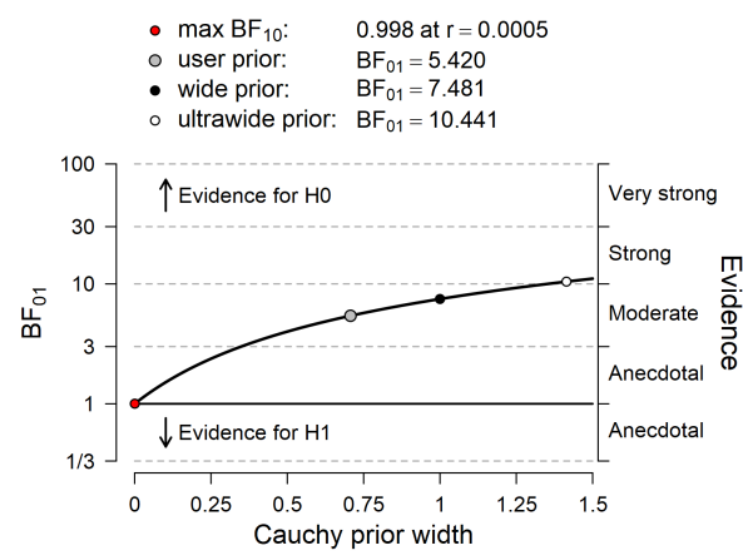




\section{Scatterplot of the Distribution of Key Presses in Experiment 3}

Figure 4.3. Ratio of x- and n-key presses per block (Action Block, Operant Action Block, Operant Effect Block)

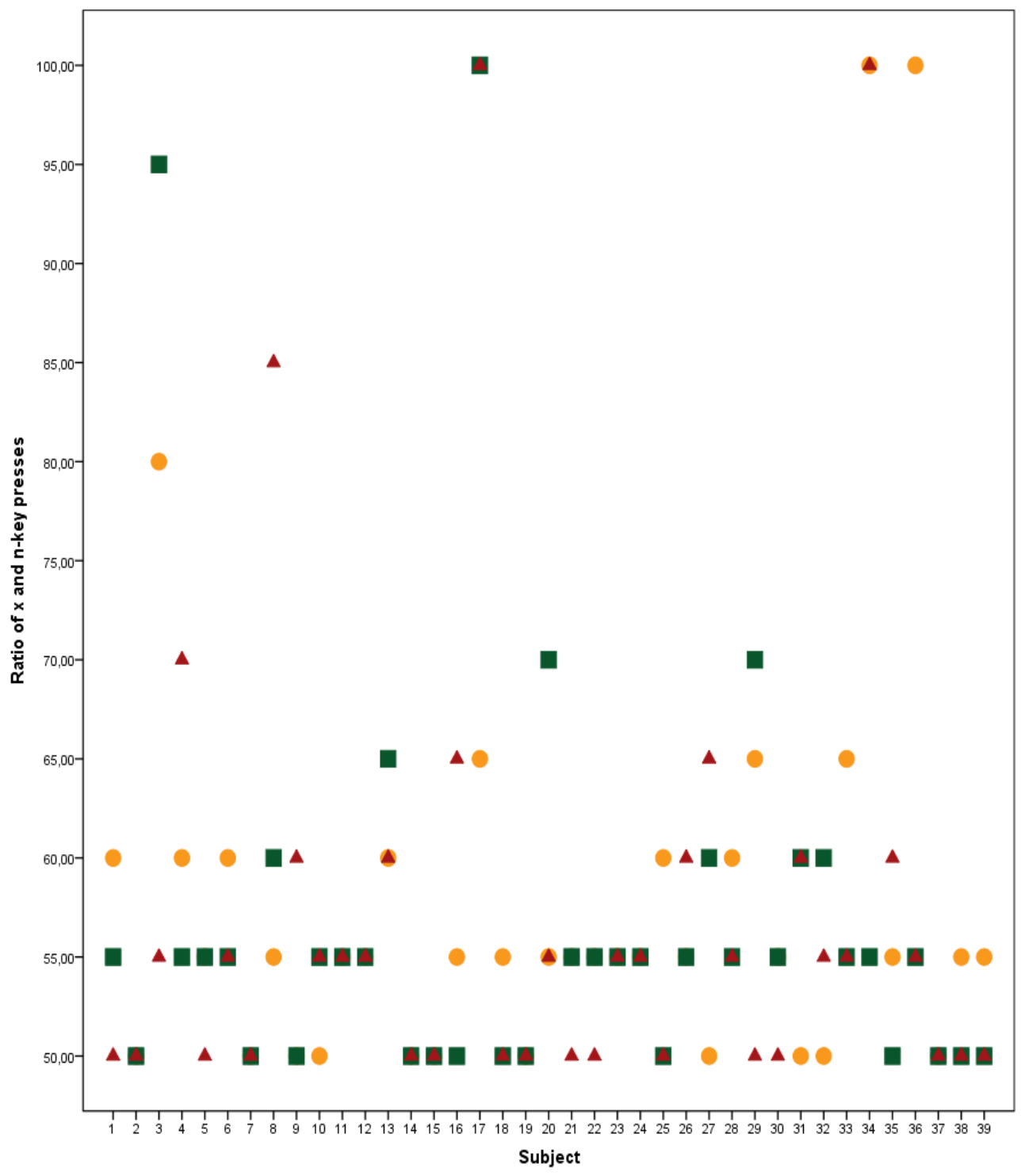

Action Block

Operant Action Block

- Operant Effect Block Subject 


\section{Contributions}

\section{Chapter 1}

Author Antusch wrote the first draft, author Aarts edited the manuscript.

\section{Chapter 2}

Authors Antusch, Custers and Aarts conceived the idea and planned the experiment. Author Antusch programmed the experiment, supervised data collection and analyzed and interpreted the data. Authors Marien aided in data interpretation. Author Antusch wrote the first draft of the manuscript. Authors Custers and Aarts edited the manuscript, author Marien provided useful comments.

\section{Chapter 3}

Authors Antusch, Custers and Aarts conceived the idea and planned the experiment. Author Antusch programmed the experiment, supervised data collection, analyzed and interpreted the data. Author Marien aided in programming. Author Custers aided in data interpretation. Author Antusch wrote the first draft of the manuscript. Author Aarts edited the manuscript, authors Custers and Marien provided useful comments.

\section{Chapter 4}

Authors Antusch, Custers and Aarts conceived the idea and planned the experiment. Author Antusch programmed the experiment, supervised data collection, analyzed and interpreted the data. Author Marien aided in programming. Author Antusch wrote the first draft of the manuscript. Author Aarts edited the manuscript, authors Custers and Marien provided useful comments. 


\section{Chapter 5}

Authors Antusch, Custers and Aarts conceived the idea and planned the experiment. Author Antusch collected, analyzed and interpreted the data. Author Custers aided in the interpretation of the results. Author Antusch wrote the first draft of the manuscript. Authors Aarts and Custers edited the manuscript. 
Dutch Summary

(Nederlandse Samenvatting) 
Nederlandse samenvatting

Intentioneel handelen en het uitvoeren van acties is een essentieel onderdeel van menselijk gedrag. Door onze acties kunnen we de wereld manipuleren en effecten veroorzaken die in overeenstemming zijn met onze doelen. Dit vermogen tot intentioneel gedrag is niet alleen belangrijk voor het uitvoeren van gemotiveerd gedrag maar verandert ook onze waarneming. Intentioneel gedrag zorgt namelijk ervoor dat we een gevoel van controle over onze acties ervaren en de effecten ervan. Dit gevoel wordt ook wel eens sense of agency genoemd. Het ervaren van een sense of agency blijkt een heel belangrijke rol te spelen in het correct ervaren van onszelf als intentionele individuen. Als de ervaring van agency verstoord is, kan dit tot ernstige problemen leiden. Het lijkt er bijvoorbeeld op dat mensen op het autisme-spectrum en mensen die psychose-gevoelig zijn een verstoorde waarneming van hun acties en de effecten ervan kunnen hebben, wat ertoe leidt dat hun ervaring van de sense of agency afwijkt. Het begrijpen van het ontstaan van sense of agency is van theoretisch en praktisch belang.

Een veelbelovende manier om sense of agency te bestuderen is door het subjectief waargenomen tijdsinterval tussen acties en effecten te meten. Als we een intentionele actie uitvoeren (bijv. een knop indrukken) om een effect te veroorzaken (bijv. een toon), blijken we die twee gebeurtenissen als dichter bij elkaar liggend in tijd waar te nemen. De actie en het daaropvolgende effect worden dus als het ware naar elkaar toegetrokken. Dit fenomeen wordt intentional binding genoemd vanwege het feit dat het effect alleen optreedt als de actie intentioneel is (Haggard et al., 2002b), maar niet als deze afgedwongen wordt door bijvoorbeeld het kunstmatig indrukken van de vinger van de proefpersoon. In dat geval wordt het tijdsinterval tussen actie en effect juist als wijder waargenomen, en lijken actie en effect elkaar soms juist af te stoten. 
Deze thesis richt zich op de vraag of intenties nu echt de cruciale component zijn voor het ontstaan van het temporal binding effect, of dat andere factoren die hier doorgaans mee gepaard gaan dit effect kunnen verklaren. We hebben hiervoor eerst de recente literatuur op het gebied van intentional binding geanalyseerd om een overzicht te krijgen van de factoren die hierin een rol zouden kunnen spelen. Daarnaast hebben we in het empirische gedeelte van deze thesis systematisch onderzocht welke ingrediënten toegevoegd moeten worden om de subjectieve waarneming van twee opeenvolgende stimuli in de tijd naar elkaar toe te laten trekken.

Het onderzoek beschreven in deze thesis toont aan dat twee opeenvolgende auditieve stimuli elkaar lijken af te stoten in de tijd, zelfs na een langdurige leerfase, hoewel deze afstoting iets verminderd lijkt te worden als mensen een causale relatie zien tussen de twee gebeurtenissen. Hetzelfde effect werd aangetoond wanneer een tactiele stimulus opgevolgd werd door een auditieve stimulus, zelfs wanneer men deze tactiele stimulus kon anticiperen en het specifieke effect van de actie kon voorspellen. Terwijl intenties dus toch cruciaal lijken voor intentional binding, lieten verdere studies zien dat het beperken van controle van intentionele acties geen effect had op het intentional binding effect, hoewel dit duidelijk effect had op de gerapporteerde waargenomen autonomie.

Deze resultaten bieden nieuwe inzichten in de rol van intentionele actie in het gevoel van controle en de sense of agency. Anders dan door sommige modellen verondersteld, blijkt dat intenties toch een cruciale component zijn voor temporal binding. Opmerkelijk is dat het ondermijnen van autonomie niet ertoe leidt dat het intentional binding effect verandert. Daartegenover staat dat acties, die met een sterkere intentie worden uitgevoerd, een sterker intentional binding effect veeroorzaken. Het lijkt dus dat motor-predictie noodzakelijk is voor het ontstaan van de integratie van actie en effect in de waarneming, maar dat intentionele processen dit proces nog verder kunnen versterken. Deze bevindingen dragen bij aan het 
begrijpen van de rol van intentionele acties in de ervaring van controle over onze acties en effecten daarvan in de buitenwereld. Toekomstig onderzoek zou deze inzichten kunnen inzetten om interventies tot gedragsverandering te creëren, of te onderzoeken hoe de sense of agency in de afwezigheid van motor-signalen kan ontstaan. 


\section{References}

Aarts, H. (2012). Goals, motivated social cognition and behavior. The SAGE handbook of social cognition, 75-79.Aarts, H., \& van den Bos, K. (2011). On the foundations of beliefs in free will: Intentional binding and unconscious priming in selfagency. Psychological Science, 22(4), 532-537.

Aarts, H., Bijleveld, E., Custers, R., Dogge, M., Deelder, M., Schutter, D., \& van Haren, N. E. (2012). Positive priming and intentional binding: Eye-blink rate predicts reward information effects on the sense of agency. Social Neuroscience, 7(1), 105-112.

Aarts, H., \& Custers, R. (2009). Habit, action, and consciousness. In Banks, W. P. (Ed.), Encyclopedia of consciousness (Vol. 1, pp. 315-328). Oxford, England: Elsevier.

Aarts, H., Custers, R., \& Marien, H. (2008a). Preparing and motivating behavior outside of awareness. Science, 319(5870), 1639-1639.

Aarts, H., Custers, R., \& Wegner, D. M. (2005). On the inference of personal authorship: Enhancing experienced agency by priming effect information. Consciousness and Cognition, 14(3), 439-458.

Aarts, H., Dijksterhuis, A., Dik, G. (2008b). Goal contagion: Inferring goals from others' actions - and what it leads to. In J.Y. Shah \& W. Gardner (Eds.), Handbook of motivation science. Guilford.

Aarts, H., Paulussen, T., \& Schaalma, H. (1997). Physical exercise habit: on the conceptualization and formation of habitual health behaviours. Health Education Research, 12(3), 363-374. 
Ajzen, I. (1991). The theory of planned behavior. Organizational Behavior and Human Decision Processes, 50(2), 179-211.

Ajzen, I., \& Fishbein, M. (1977). Attitude-behavior relations: A theoretical analysis and review of empirical research. Psychological Bulletin, 84(5), 888.

Antusch, S., Aarts, H., \& Custers, R. (2019). The Role of Intentional Strength in Shaping the Sense of Agency. Frontiers in Psychology, 10, 1124.

Antusch, S., Custers, R., Marien, H., \& Aarts, H., a, (under review). Sense of Agency in the Absence of Motor Movement: An Investigation into Temporal Binding of Tactile Sensations and Auditory Effects.

Antusch, S., Custers, R., Marien, H., \& Aarts, H., b, (under review). Intentional Action and Limitation of Personal Autonomy. Do Restrictions of Action Selection Decrease the Sense of Agency?

Antusch, S., Custers, R., Marien, H., \& Aarts, H. (2020). Intentionality and temporal binding: Do causality beliefs increase the perceived temporal attraction between events?. Consciousness and Cognition, 77, 102835.

Aschersleben, G., \& Prinz, W. (1995). Synchronizing actions with events: The role of sensory information. Perception \& Psychophysics, 57(3), 305-317.

Aston-Jones, G., \& Cohen, J. D. (2005). An integrative theory of locus coeruleusnorepinephrine function: adaptive gain and optimal performance. Annu. Rev. Neurosci., 28, 403-450.

Baldwin, D. A., \& Baird, J. A. (2001). Discerning intentions in dynamic human action. Trends in Cognitive Sciences, 5(4), 171-178. 
Bandura, A. (1977). Self-efficacy: toward a unifying theory of behavioral change. Psychological Review, 84(2), 191.

Barlas, Z., \& Obhi, S. (2013). Freedom, choice, and the sense of agency. Frontiers in Human Neuroscience, 7, 514.

Bartlett, J. E. (2018, March 14). An Introduction to JASP: A Free and User-Friendly Statistics Package. Retrieved from osf.io/7x $8 \mathrm{hj}$

Bentham, J. (1789). An Introduction to the Principles of Morals and Legislation, London, T. Volume, DOI.

Bergstrom-Lehtovirta, J., Coyle, D., Knibbe, J., \& Hornbæk, K. (2018, April). I Really did That: Sense of Agency with Touchpad, Keyboard, and On-skin Interaction. In Proceedings of the 2018 CHI Conference on Human Factors in Computing Systems (p. 378). ACM.

Bijleveld, E., \& Aarts, H. (2014). A psychological perspective on money. In The psychological science of money (pp. 3-19). Springer.

Blakemore, S. J., Frith, C. D., \& Wolpert, D. M. (1999). Spatio-temporal prediction modulates the perception of self-produced stimuli. Journal of Cognitive Neuroscience, 11(5), 551-559.

Blakemore, S. J., Wolpert, D. M., \& Frith, C. D. (2002). Abnormalities in the awareness of action. Trends in Cognitive Sciences, 6(6), 237-242.

Blakemore, S. J., Wolpert, D., \& Frith, C. (2000). Why can't you tickle yourself?. Neuroreport, 11(11), R11-R16. 
Borhani, K., Beck, B., \& Haggard, P. (2017). Choosing, doing, and controlling: implicit sense of agency over somatosensory events. Psychological Science, 28(7), 882-893.

Brandstätter, V., Lengfelder, A., \& Gollwitzer, P. M. (2001). Implementation intentions and efficient action initiation. Journal of Personality and Social Psychology, 81(5), 946.

Brass, M., \& Haggard, P. (2008). The what, when, whether model of intentional action. The Neuroscientist, 14(4), 319-325.

Bratman, M. E. (1999). Faces of intention: Selected essays on intention and agency. Cambridge University Press.

Brentano, F. (2014). Psychology from an empirical standpoint. Routledge.

Buehner, M. J. (2012). Understanding the past, predicting the future: causation, not intentional action, is the root of temporal binding. Psychological Science, 23(12), $1490-1497$.

Buehner, M. J., \& Humphreys, G. R. (2009). Causal binding of actions to their effects. Psychological Science, 20(10), 1221-1228.

Buehner, M. J., \& Humphreys, G. R. (2010). Causal contraction: spatial binding in the perception of collision events. Psychological Science, 21(1), 44-48.

Cameron, J., \& Pierce, W. D. (1994). Reinforcement, reward, and intrinsic motivation: A meta-analysis. Review of Educational Research, 64(3), 363-423.

Caspar, E. A., Christensen, J. F., Cleeremans, A., \& Haggard, P. (2016). Coercion changes the sense of agency in the human brain. Current Biology, 26(5), 585-592. 
Caspar, E. A., Vuillaume, L., Magalhães De Saldanha da Gama, P. A., \& Cleeremans, A. (2017). The influence of (dis) belief in free will on immoral behavior. Frontiers in Psychology, 8, 20.

Conradt, S. (2011, September 2). Eine Frage von Recht und Gerechtigkeit. Retrieved from: https://www.deutschlandfunk.de/eine-frage-von-recht-und gerechtigkeit.871.de.html?dram:article_id=127444

Cornelio Martinez, P. I., De Pirro, S., Vi, C. T., \& Subramanian, S. (2017, May). Agency in mid-air interfaces. In Proceedings of the 2017 CHI Conference on Human Factors in Computing Systems (pp. 2426-2439). ACM.

Cornelio Martinez, P. I., Maggioni, E., Hornbæk, K., Obrist, M., \& Subramanian, S. (2018, April). Beyond the Libet clock: modality variants for agency measurements. In Proceedings of the 2018 CHI Conference on Human Factors in Computing Systems (p. 541). ACM.

Coyle, D., Moore, J., Kristensson, P. O., Fletcher, P., \& Blackwell, A. (2012, May). I did that! Measuring users' experience of agency in their own actions. In Proceedings of the SIGCHI Conference on Human Factors in Computing Systems (pp. 2025-2034). ACM.

Cravo, A. M., Claessens, P. M., \& Baldo, M. V. (2009). Voluntary action and causality in temporal binding. Experimental Brain Research, 199(1), 95-99.

Damen, T. G., Müller, B. C., van Baaren, R. B., \& Dijksterhuis, A. (2015). Re-examining the agentic shift: The sense of agency influences the effectiveness of (self) persuasion. PloS One, 10(6), e0128635. 
Damen, T. G., Van Baaren, R. B., Brass, M., Aarts, H., \& Dijksterhuis, A. (2015). Put your plan into action: The influence of action plans on agency and responsibility. Journal of Personality and Social Psychology, 108(6), 850.

Decety, J., \& Grèzes, J. (2006). The power of simulation: imagining one's own and other's behavior. Brain Research, 1079(1), 4-14.

Deci, E. L., Koestner, R., \& Ryan, R. M. (1999). A meta-analytic review of experiments examining the effects of extrinsic rewards on intrinsic motivation. Psychological Bulletin, 125(6), 627.

Deci, E. L., \& Ryan, R. M. (1985). Intrinsic motivation and self-determination in human behavior. Plenum.

Dennett, D. C. (1993). Consciousness Explained. Penguin UK.

Dennett, D. C. (2004). Freedom evolves. Penguin UK.

Desantis, A., Hughes, G., \& Waszak, F. (2012). Intentional binding is driven by the mere presence of an action and not by motor prediction. PLoS One, 7(1), e29557.

Desantis, A., Roussel, C., \& Waszak, F. (2011). On the influence of causal beliefs on the feeling of agency. Consciousness and Cognition, 20(4), 1211-1220.

Deschrijver, E., Wiersema, J. R., \& Brass, M. (2016). The interaction between felt touch and tactile consequences of observed actions: an action-based somatosensory congruency paradigm. Social Cognitive and Affective Neuroscience, 11(7), 1162-1172.

Dewey, J. A., \& Knoblich, G. (2014). Do implicit and explicit measures of the sense of agency measure the same thing?. PloS One, 9(10), e110118. 
Dijksterhuis, A., \& Aarts, H. (2012). Control, consciousness, and agency. Aarts, H., Elliot, A. (Eds.). Goal-directed behavior. (pp. 301 - 324). Taylor \& Francis.

Dogge, M., Custers, R., \& Aarts, H. (2019a). Moving forward: On the limits of motor-based forward models. Trends in Cognitive Sciences, 23, 743-753.

Dogge, M., Hofman, D., Boersma, M., Dijkerman, H. C., \& Aarts, H. (2014). Cortical information flow during inferences of agency. Frontiers in Human Neuroscience, 8, 609.

Dogge, M., Hofman, D., Custers, R., \& Aarts, H. (2019b). Exploring the role of motor and non-motor predictive mechanisms in sensory attenuation: Perceptual and neurophysiological findings. Neuropsychologia, 124, 216-225.

Dogge, M., Schaap, M., Custers, R., Wegner, D. M., \& Aarts, H. (2012). When moving without volition: implied self-causation enhances binding strength between involuntary actions and effects. Consciousness and Cognition, 21(1), 501-506.

Dreisbach, G., \& Goschke, T. (2004). How positive affect modulates cognitive control: reduced perseveration at the cost of increased distractibility. Journal of Experimental Psychology: Learning, Memory, and Cognition, 30(2), 343.

Eagleman, D. M., \& Holcombe, A. O. (2002). Causality and the perception of time. Trends in Cognitive Sciences, 6(8), 323-325.

Ehrsson, H. H., Spence, C., \& Passingham, R. E. (2004). That's my hand! Activity in premotor cortex reflects feeling of ownership of a limb. Science, 305(5685), 875-877. 
Eitam, B., \& Higgins, E. T. (2010). Motivation in mental accessibility: Relevance of a representation (ROAR) as a new framework. Social and Personality Psychology Compass, 4(10), 951-967.

Engbert, K., \& Wohlschläger, A. (2007). Intentions and expectations in temporal binding. Consciousness and Cognition, 16(2), 255-264.

Ernst, M. O. (2007). Learning to integrate arbitrary signals from vision and touch. Journal of Vision, 7(5), 7-7.

Faul, F., Erdfelder, E., Lang, A.-G., \& Buchner, A. (2007). G*Power 3: A flexible statistical power analysis program for the social, behavioral, and biomedical sciences. Behavior Research Methods, 39, 175-191.

Fishbein, M., \& Ajzen, I. (1975). Belief. Attitude, Intention and Behavior: An Introduction to Theory and Research, 578.

Frankfurt, H. (1971). Freedom of the Will and the Concept of a Person. Journal of Philosophy, 68.

Frith, C. (2002). Attention to action and awareness of other minds. Consciousness and Cognition, 11(4), 481-487.

Frith, C. D., Blakemore, S. J., \& Wolpert, D. M. (2000). Abnormalities in the awareness and control of action. Philosophical Transactions of the Royal Society of London. Series B: Biological Sciences, 355(1404), 1771-1788.

Gallagher, S. (2000). Philosophical conceptions of the self: implications for cognitive science. Trends in Cognitive Sciences, 4(1), 14-21. 
Gneezy, U., Meier, S., \& Rey-Biel, P. (2011). When and why incentives (don't) work to modify behavior. Journal of Economic Perspectives, 25(4), 191-210.

Gollwitzer, P. M. (1993). Goal achievement: The role of intentions. European Review of Social Psychology, 4(1), 141-185.

Gollwitzer, P. M. (1999). Implementation intentions: Strong effects of simple plans. American Psychologist, 54(7), 493.

Greenwald, A. G. (1970). Sensory feedback mechanisms in performance control: with special reference to the ideo-motor mechanism. Psychological Review, 77(2), 73.

Grotheer, M., \& Kovács, G. (2014). Repetition probability effects depend on prior experiences. Journal of Neuroscience, 34(19), 6640-6646.

Haggard, P. (2017). Sense of agency in the human brain. Nature Reviews Neuroscience, 18(4), 196.

Haggard, P., \& Clark, S. (2003). Intentional action: Conscious experience and neural prediction. Consciousness and Cognition, 12(4), 695-707.

Haggard, P., \& Tsakiris, M. (2009). The experience of agency: Feelings, judgments, and responsibility. Current Directions in Psychological Science, 18(4), 242-246.

Haggard, P., Aschersleben, G., Gehrke, J., \& Prinz, W. (2002a). Action, binding, and awareness. W. Prinz, B. Hommel, (Eds.), Common mechanisms in perception and action: Attention and performance Vol. XIX (pp. 266-285). Oxford University Press.

Haggard, P., Clark, S., \& Kalogeras, J. (2002b). Voluntary action and conscious awareness. Nature Neuroscience, 5(4), 382-385. 
Haggard, P., Martin, F., Taylor-Clarke, M., Jeannerod, M., \& Franck, N. (2003). Awareness of action in schizophrenia. Neuroreport, 14(7), 1081-1085.

Halpern, D. (2015). Inside the nudge unit: How small changes can make a big difference. Random House.

Harris, C., Fiedler, K., Marien, H., \& Custers, R. (2020). Biased preferences through exploitation: How initial biases are consolidated in reward-rich environments. Journal of Experimental Psychology: General.

Hommel, B., Müsseler, J., Aschersleben, G., \& Prinz, W. (2001). The theory of event coding (TEC): A framework for perception and action planning. Behavioral and Brain Sciences, 24(5), 849-878.

Hsu, Y. F., Le Bars, S., Hämäläinen, J. A., \& Waszak, F. (2015). Distinctive representation of mispredicted and unpredicted prediction errors in human electroencephalography. Journal of Neuroscience, 35(43), 14653-14660.

Hughes, G., Desantis, A., \& Waszak, F. (2013). Mechanisms of intentional binding and sensory attenuation: The role of temporal prediction, temporal control, identity prediction, and motor prediction. Psychological Bulletin, 139(1), 133.

Hume, D. (1888). A treatise of human nature (Selby-Bigge, L.A., Ed.). Clarendon Press. (Original work published 1739)

Humphreys, G. R., \& Buehner, M. J. (2009). Magnitude estimation reveals temporal binding at super-second intervals. Journal of Experimental Psychology: Human Perception and Performance, 35(5), 1542. 
Imaizumi, S., \& Tanno, Y. (2019). Intentional binding coincides with explicit sense of agency. Consciousness and Cognition, 67, 1-15.

Iwatsuki, T., Shih, H. T., Abdollahipour, R., \& Wulf, G. (2019). More bang for the buck: autonomy support increases muscular efficiency. Psychological Research, 1-7.

Jacobsen, T., \& Schröger, E. (2001). Is there pre-attentive memory-based comparison of pitch?. Psychophysiology, 38(4), 723-727.

James, W. (1890). The perception of reality. Principles of Psychology, 2, 283-324.

JASP Team (2018). JASP (Version 0.9)[Computer software].

Jo, H. G., Hinterberger, T., Wittmann, M., \& Schmidt, S. (2015). Do meditators have higher awareness of their intentions to act?. Cortex, 65, 149-158.

Karsh, N., \& Eitam, B. (2015). I control therefore I do: Judgments of agency influence action selection. Cognition, 138, 122-131.

Karsh, N., Eitam, B., Mark, I., \& Higgins, E. T. (2016). Bootstrapping agency: How controlrelevant information affects motivation. Journal of Experimental Psychology: General, 145(10), 1333.

Kirsch, W., Kunde, W., \& Herbort, O. (2019). Intentional binding is unrelated to action intention. Journal of Experimental Psychology: Human Perception and Performance, 45(3), 378.

Koestner, R. (2008). Reaching one's personal goals: A motivational perspective focused on autonomy. Canadian Psychology/Psychologie Canadienne, 49(1), 60.

Korsgaard, C. M. (2009). Self-constitution: Agency, identity, and integrity. OUP Oxford. 
Kühn, S., Brass, M., \& Haggard, P. (2013). Feeling in control: Neural correlates of experience of agency. Cortex, 49(7), 1935-1942.

Lange, K. (2009). Brain correlates of early auditory processing are attenuated by expectations for time and pitch. Brain and Cognition, 69(1), 127-137.

Libet, B. (1985). Unconscious cerebral initiative and the role of conscious will in voluntary action. Behavioral and Brain Sciences, 8(4), 529-539.

Libet, B., Gleason, C. A., Wright, E. W., \& Pearl, D. K. (1983). Time of conscious intention to act in relation to onset of cerebral activity (readiness-potential). In Neurophysiology of Consciousness (pp. 249-268). Birkhäuser.

Locke, J. 1823. The Works of John Locke. London: Printed for T. Tegg (10 volumes)

Locke, E., \& Latham, G. (1994). Goal-setting theory. Organizational behavior 1. Essential Theories of Motivation and Leadership, 159-183. Routledge.

Locke, E. A., \& Latham, G. P. (2019). The development of goal setting theory: A half century retrospective. Motivation Science, 5(2), 93.

Lucas, C. G., \& Griffiths, T. L. (2010). Learning the form of causal relationships using hierarchical Bayesian models. Cognitive Science, 34(1), 113-147.

Lush, P., Parkinson, J., \& Dienes, Z. (2016). Illusory temporal binding in meditators. Mindfulness, 7(6), 1416-1422.

Lynn, M. T., Muhle-Karbe, P. S., Aarts, H., \& Brass, M. (2014). Priming determinist beliefs diminishes implicit (but not explicit) components of self-agency. Frontiers in Psychology, 5, 1483. 
Marien, H., Custers, R., \& Aarts, H. (2019). Studying Human Habits in Societal Context: Examining Support for a Basic Stimulus-Response Mechanism. Current Directions in Psychological Science, 28(6), 614-618.

Marteau, T. M., Ogilvie, D., Roland, M., Suhrcke, M., \& Kelly, M. P. (2011). Judging nudging: can nudging improve population health?. Bmj, 342 .

Maselli, A., Kilteni, K., López-Moliner, J., \& Slater, M. (2016). The sense of body ownership relaxes temporal constraints for multisensory integration. Scientific Reports, 6, 30628.

Mates, J., Radil, T., \& Pöppel, E. (1992). Cooperative tapping: Time control under different feedback conditions. Perception \& Psychophysics, 52(6), 691-704.

Milgram, S. (1974). Obedience to authority: An experimental view. Harper \& Row.

Mill, J. S. (1861 / 2001) Utilitarianism. Hackett Publishing.

Miller, D. T., \& Ross, M. (1975). Self-serving biases in the attribution of causality: Fact or fiction?. Psychological Bulletin, 82(2), 213.

Moore, J., \& Haggard, P. (2008). Awareness of action: Inference and prediction. Consciousness and Cognition, 17(1), 136-144.

Moore, J. W., Middleton, D., Haggard, P., \& Fletcher, P. C. (2012). Exploring implicit and explicit aspects of sense of agency. Consciousness and Cognition, 21(4), 1748-1753.

Moore, J. W., \& Obhi, S. S. (2012). Intentional binding and the sense of agency: a review. Consciousness and Cognition, 21(1), 546-561.

Moore, J. W., Schneider, S. A., Schwingenschuh, P., Moretto, G., Bhatia, K. P., \& Haggard, P. (2010). Dopaminergic medication boosts action-effect binding in Parkinson's disease. Neuropsychologia, 48(4), 1125-1132. 
Moore, J. W., Teufel, C., Subramaniam, N., Davis, G., \& Fletcher, P. C. (2013). Attribution of intentional causation influences the perception of observed movements: behavioral evidence and neural correlates. Frontiers in Psychology, 4, 23.

Moore, J. W., Wegner, D. M., \& Haggard, P. (2009). Modulating the sense of agency with external cues. Consciousness and Cognition, 18(4), 1056-1064.

Moreton, J., Callan, M. J., \& Hughes, G. (2017). How much does emotional valence of action outcomes affect temporal binding?. Consciousness and Cognition, 49, 25-34.

Moretto, G., Walsh, E., \& Haggard, P. (2011). Experience of agency and sense of responsibility. Consciousness and Cognition, 20(4), 1847-1854.

Obhi, S. S., \& Hall, P. (2011). Sense of agency and intentional binding in joint action. Experimental Brain Research, 211(3-4), 655.

Pacherie, E. (2006). Toward a dynamic theory of intentions Does Consciousness Cause Behavior? An Investigation of the Nature of Volition (pp. 145-167). MIT Press

Passingham, R. E., Bengtsson, S. L., \& Lau, H. C. (2010). Medial frontal cortex: from selfgenerated action to reflection on one's own performance. Trends in Cognitive Sciences, 14(1), 16-21.

Pfister, R., Kiesel, A., \& Hoffmann, J. (2011). Learning at any rate: Action-effect learning for stimulus-based actions. Psychological Research, 75(1), 61-65.

Pollack, D. (2004). Support for democracy in Eastern and Western Germany: an attempt to explain the differences. European Journal of Sociology/Archives Européennes de Sociologie, 45(2), 257-272. 
Poonian, S. K., \& Cunnington, R. (2013). Intentional binding in self-made and observed actions. Experimental Brain Research, 229(3), 419-427.

Prikken, M., van der Weiden, A., Baalbergen, H., Hillegers, M. H., Kahn, R. S., Aarts, H., \& van Haren, N. E. (2019). Multisensory integration underlying body-ownership experiences in schizophrenia and offspring of patients: a study using the rubber hand illusion paradigm. Journal of Psychiatry \& Neuroscience: JPN, 44(3), 177.

Prinz, W. (1997). Perception and action planning. European Journal of Cognitive Psychology, 9(2), 129-154.

Quintana, D. S., \& Williams, D. R. (2018). Bayesian alternatives for common null-hypothesis significance tests in psychiatry: a non-technical guide using JASP. BMC Psychiatry, 18(1), 178.

Renes, R. A., van Haren, N. E., Aarts, H., \& Vink, M. (2015). An exploratory fMRI study into inferences of self-agency. Social Cognitive and Affective Neuroscience, 10(5), 708-712.

Renes, R. A., Vink, M., van der Weiden, A., Prikken, M., Koevoets, M. G., Kahn, R. S., ... \& van Haren, N. E. (2016). Impaired frontal processing during agency inferences in schizophrenia. Psychiatry Research: Neuroimaging, 248, 134-141.

Rigby, C. S., Deci, E. L., Patrick, B. C., \& Ryan, R. M. (1992). Beyond the intrinsic-extrinsic dichotomy: Self-determination in motivation and learning. Motivation and Emotion, 16(3), 165-185.

Ruess, M., Thomaschke, R., \& Kiesel, A. (2017). The time course of intentional binding. Attention, Perception, \& Psychophysics, 79(4), 1123-1131. 
Ruess, M., Thomaschke, R., \& Kiesel, A. (2018). Intentional binding of visual effects. Attention, Perception, \& Psychophysics, 80(3), 713-722.

Ruys, K. I., \& Aarts, H. (2012). I didn't mean to hurt you! Unconscious origins of experienced self-agency over others' emotions. Emotion, 12(1), 132.

Ryan, R. M., \& Deci, E. L. (2006). Self-regulation and the problem of human autonomy: Does psychology need choice, self-determination, and will?. Journal of Personality, 74(6), 1557-1586.

Saito, N., Takahata, K., Murai, T., \& Takahashi, H. (2015). Discrepancy between explicit judgement of agency and implicit feeling of agency: Implications for sense of agency and its disorders. Consciousness and Cognition, 37, 1-7.

Schultz, W. (2006). Behavioral theories and the neurophysiology of reward. Annu. Rev. Psychol., 57, 87-115.

Schupp, H. T., Öhman, A., Junghöfer, M., Weike, A. I., Stockburger, J., \& Hamm, A. O. (2004). The facilitated processing of threatening faces: an ERP analysis. Emotion, 4(2), 189.

Schwarz, K. A., Weller, L., Klaffehn, A. L., \& Pfister, R. (2019). The effects of action choice on temporal binding, agency ratings, and their correlation. Consciousness and Cognition, 75, 102807.

Searle, J. R.(1983). Intentionality: An essay in the philosophy of mind. Cambridge University Press.

Serences, J. T., \& Yantis, S. (2006). Selective visual attention and perceptual coherence. Trends in Cognitive Sciences, 10(1), 38-45. 
Shamma, S. A., Elhilali, M., \& Micheyl, C. (2011). Temporal coherence and attention in auditory scene analysis. Trends in Neurosciences, 34(3), 114-123.

Sheeran, P. (2002). Intention-behavior relations: a conceptual and empirical review. European Review of Social Psychology, 12(1), 1-36.

Shimi, A., \& Logie, R. H. (2018). Feature binding in short-term memory and long-term learning. Quarterly Journal of Experimental Psychology, 1747021818807718.

Shin, Y. K., Proctor, R. W., \& Capaldi, E. J. (2010). A review of contemporary ideomotor theory. Psychological Bulletin, 136(6), 943.

Soto-Faraco, S., Spence, C., \& Kingstone, A. (2004). Congruency effects between auditory and tactile motion: extending the phenomenon of cross-modal dynamic capture. Cognitive, Affective, \& Behavioral Neuroscience, 4(2), 208-217.

Sperduti, M., Pieron, M., Leboyer, M., \& Zalla, T. (2014). Altered pre-reflective sense of agency in autism spectrum disorders as revealed by reduced intentional binding. Journal of Autism and Developmental Disorders, 44(2), 343-352.

Sunstein, C. R., \& Thaler, R. H. (2003). Libertarian paternalism is not an oxymoron. The University of Chicago Law Review, 1159-1202.

Suzuki, K., Lush, P., Seth, A. K., \& Roseboom, W. (2019). Intentional binding without intentional action. Psychological Science, 30(6), 842-853.

Synofzik, M., Vosgerau, G., \& Lindner, A. (2009). Me or not me-An optimal integration of agency cues?. Consciousness and Cognition, 18(4), 1065-1068. 
Synofzik, M., Vosgerau, G., \& Newen, A. (2008). Beyond the comparator model: a multifactorial two-step account of agency. Consciousness and Cognition, 17(1), 219239.

Takahata, K., Takahashi, H., Maeda, T., Umeda, S., Suhara, T., Mimura, M., \& Kato, M. (2012). It's not my fault: postdictive modulation of intentional binding by monetary gains and losses. PloS One, 7(12), e53421.

Tanaka, T., \& Kawabata, H. (2019). Sense of agency is modulated by interactions between action choice, outcome valence, and predictability. Current Psychology, 1-12.

Tanaka, T., Matsumoto, T., Hayashi, S., Takagi, S., \& Kawabata, H. (2019). What Makes Action and Outcome Temporally Close to Each Other: A Systematic Review and Meta-Analysis of Temporal Binding. Timing \& Time Perception, 7(3), 189-218.

Thanopoulos, V., Psarou, E., \& Vatakis, A. (2018). Robust intentional binding for causallylinked sequences of naturalistic events but not for abstract event sequences. Acta Psychologica, 190, 159-173.

Tombor, I., \& Michie, S. F. (2017). Methods of health behavior change. Oxford Research Encyclopedias-Psychology.

Tummers, L. (2019). Public policy and behavior change. Public Administration Review, 79(6), 925-930.

Turk-Browne, N. B., Scholl, B. J., \& Chun, M. M. (2008). Babies and brains: habituation in infant cognition and functional neuroimaging. Frontiers In Human Neuroscience, 2, 16. 
Vallacher, R. R., \& Wegner, D. M. (1987). What do people think they're doing? Action identification and human behavior. Psychological Review, 94(1), 3.

van der Weiden, A., Aarts, H., \& Ruys, K. I. (2010). Reflecting on the action or its outcome: Behavior representation level modulates high level outcome priming effects on selfagency experiences. Consciousness and Cognition, 19(1), 21-32.

van der Weiden, A., Aarts, H., \& Ruys, K. I. (2011). Prime and probability: Causal knowledge affects inferential and predictive effects on self-agency experiences. Consciousness and Cognition, 20(4), 1865-1871.

van Inwagen, P. (1975). The incompatibility of free will and determinism. Philosophical Studies, 27(3), 185-199.

Vastano, R., Deschrijver, E., Pozzo, T., \& Brass, M. (2018). Temporal binding effect in the action observation domain: Evidence from an action-based somatosensory paradigm. Consciousness and Cognition, 60, 1-8.

Voss, M., Moore, J., Hauser, M., Gallinat, J., Heinz, A., \& Haggard, P. (2010). Altered awareness of action in schizophrenia: a specific deficit in predicting action consequences. Brain, 133(10), 3104-3112.

Walter, S., \& Meier, B. (2014). How important is importance for prospective memory? A review. Frontiers in Psychology, 5, 657.

Wang, Y., Damen, T. G., \& Aarts, H. (2017). Uncovering effects of self-control and stimulusdriven action selection on the sense of agency. Consciousness and Cognition, 55, 245253.

Wegner, D. M. (2002). The illusion of free will. MIT Press. 
Wegner, D. M. (2003). The mind's best trick: how we experience conscious will. Trends in Cognitive Sciences, 7(2), 65-69.

Weiskrantz, L., Elliott, J., \& Darlington, C. (1971). Preliminary observations on tickling oneself. Nature, 230(5296), 598-599.

Wen, W., Yamashita, A., \& Asama, H. (2015). The influence of action-outcome delay and arousal on sense of agency and the intentional binding effect. Consciousness and Cognition, 36, 87-95.

Wenke, D., Waszak, F., \& Haggard, P. (2009). Action selection and action awareness. Psychological Research PRPF, 73(4), 602-612.

Wood, W., \& Rünger, D. (2016). Psychology of habit. Annual Review of Psychology, 67, 289314.

Yin, H. H., \& Knowlton, B. J. (2006). The role of the basal ganglia in habit formation. Nature Reviews Neuroscience, 7(6), 464-476.

Yoshie, M., \& Haggard, P. (2013). Negative emotional outcomes attenuate sense of agency over voluntary actions. Current Biology, 23(20), 2028-2032.

Zhao, K., Hu, L., Qu, F., Cui, Q., Piao, Q., Xu, H., ... \& Fu, X. (2016). Voluntary action and tactile sensory feedback in the intentional binding effect. Experimental Brain Research, 234(8), 2283-2292. 
Acknowledgements 
When we believe that we are in control of our actions and their effects, we often lose track of time - it literally seems to fly. This is expressed in the experience of a sense of agency, or in other words, the experience of being in control. While in retrospect it feels like the last 4.5 years flew by, there were moments when I doubted my own agency. Like most PhD candidates, at times I reached a point at which I was questioning whether I would ever finish this dissertation. However, by the end of it, I realized that this process, whilst producing a collection of articles that I am proud of, has also taught me lessons beyond the realms of psychology. The challenges and experiences that I faced as part of this process have helped me come a long way. Along this journey, I have had the pleasure of getting to know and receiving support from many kind and talented people. I would be remiss if I did not acknowledge their contributions in helping me complete this dissertation.

Henk, as my promoter you have been a great professional inspiration. I continue to be amazed and impressed by your fascination for research and the passion you hold for your work. Your guidance significantly shaped my ideas and gave the project a clear roadmap. Whenever I felt stuck, you helped me to get out of what I felt was a dead end. Most importantly, you were not only an academic advisor to me but also showed me support and empathy during moments of hardship.

Ruud, we go back a long time to when I was still a Research Master student working with you on my thesis-project and you were still working at the University College London. The time that I spent on the project at UCL was the beginning of my passion for the topic of agency and paved the road for my future career. You are a skilled researcher and I thoroughly enjoyed the long discussions we had about the methodology of studies. I could always count on your feedback (sometimes delayed because of your busy schedule and the three parallel appointments that you had scheduled but accidentally forgotten about) and support. It was 
also you who invoked the thought of pursuing a PhD in me. I am grateful for your and Henk's support and supervision throughout the last years.

Talking of inspiring and talented researchers, I do not want to miss the opportunity to thank all the members of Goallab (formerly known as SCAN or SPAM). The lab meetings were always a source of inspiration and interesting discussions. Tom and Hans, whenever I had a practical or theoretical question, I could turn to either of you, knowing that your input would help me further.

Spending a lot of time at work also meant long days in the office - luckily, I got to share my office with some amazing people, Myrthel and Yuru, I greatly value all the discussions we had about research and I fondly remember our conference trips. Manuel, with you joining the office, we did not only gain some much-needed calmness but also an incredibly kind and friendly mind. You truly love what you do, and I am glad that we were not only colleagues but that I now have the pleasure of calling you a friend. Then there is Chris, another bright mind whom I had the fortune of getting to know and sharing the office with. I am indebted to you for your patience in helping me with $\mathrm{R}$ when, in more than one instance, I wanted to produce customized special plots again, and of course it was nice to have another fellow German to talk to.

Finally, there is the last generation of office mates: Dan, Nil and Ilona. What a nice time we have had - you are all truly kind and compassionate people and it was my pleasure to have met you and shared the highs and lows of academia with you. I sincerely hope that you keep our office jungle alive for long.

I was lucky to do my $\mathrm{PhD}$ in a large department with a lot of other $\mathrm{PhD}$ candidates and research assistants. Many thanks to Inga, Melissa, Lianne, Onur, Jeanette, Dominique, Jonas, Nadia, Wenrui, Martine, Laurens, Anneloes, Baran, David, Peikei, Kevin, Piet, 
Femke, Miriam, and Tessa, for the PhD meetings, the motivational writing club gatherings, the fun nights out and the PhD dinners!

Then there are countless people from the support teams such as Jim, Pieter, Roy, and Monique, as well as the secretary's office who deserve an extra mention for their efforts and help!

My completion of this dissertation would have not been possible without the support of my friends and family who were always at my side. Jan, I am incredibly grateful for having you in my life, being certain that you will always have my back and help me achieve my goals. Knowing that there is someone who knows me so well and whom I can call day and night, has always been a source of energy and consolation for me. I am very thankful for your continuous support and friendship and I am glad that with Laura you have found an equally wonderful and kind partner.

Anki, while we already knew of each other's existence since our Bachelor's, it was not until a trip to Lithuania years later that we began to form a friendship. I am happy that we have stayed in touch since then - even when both our lives were stressful, and we seem to always move further away from each other again. Thank you for your extremely reflective and calm nature, which can act as a voice of reason when I am feeling lost amidst my emotions!

Lenka, I am not understating it when I say that you are one of the most genuine and loyal people I know. It makes me extremely happy that our friendship endured in spite of us having lived in different countries for most of the time and I am glad to know that you have found yourself a home in Sander and Ilya. It is with confidence that I say that I am sure that this friendship will last also now that I moved to Berlin! 
Timothy, what started with a tipsy night out in Groningen, turned into a wonderful friendship. I cherish our long conversations about everything and nothing as well as our pizza dinners in Utrecht of which I hope to have another one soon.

Whenever I needed a distraction, Ahmet, Zoltan, and Anita, you were there for me. Our dinners, movie evenings and road trips are always a delight and I look forward to many more to come! Xavier, no matter how different our worlds and values can be, I always enjoy talking to you and I value you as a loyal and honest friend.

Rashad, our conversations about philosophy, politics and culture over dinner and wine were some of my favourites - I even enjoy our heated discussions when we disagree. I consider myself lucky to know that I am always welcome at your home and dinner-table.

Tina, unbelievable that it has been seven years since we first met during the introduction to the Research Master. During that time, we grew together as friends and colleagues. Your cando attitude and the multitude of ideas that you have, have always inspired me. I fondly remember our talks about research and our private lives, the vegan dinners and the movie evenings. I hope that we meet each other again soon - whether it be in Aarhus or Berlin.

Monika, I know that you are always supportive of what I do and that you will always have an open ear for me. Our occasional flea-market strolls and museum visits were always a welcome distraction!

I would also like to express my gratitude to Thomas for your support throughout the years, Bernd for the long conversations and dinners that we had and Gaëtan who is not afraid to take on unpopular views and defend them with logic, something which I greatly admire. Nicole - not only did we share an apartment in the last two years but we also celebrated each 
other's academic achievements and supported one another when academia gave us a tough time. Thanks for being there for me during those times!

Ties, this has been a journey and a lot of lessons learnt for the both of us. Not only did you show me the world from a different perspective - one unique to you - but also mesmerized me with your incessant logic paired with a big heart and incredible empathy levels. Talking to you is always a pleasure and I am indebted to you for teaching me to believe in myself and be fearless of challenges. I think I do not need a lot of words to express my gratitude but one thing that I should not leave unacknowledged: I have been amused, sometimes mildly annoyed, but I was always immensely appreciative of the dedication with which you corrected my Dutch "word salads"!

I would like to conclude these acknowledgements by thanking my family. Julian, mittlerweile ist aus meinem "kleinen Bruder" mein "großer kleiner Bruder" geworden. Ich bin unendlich dankbar dafür, dass wir einander haben und dass wir trotz der Entfernung während der letzten Jahre noch näher zusammengewachsen sind. Wir sind beide keine Freunde großer Worte, aber ich hoffe du weißt, wie wichtig du für mich bist. Mama, Julian und ich konnten uns immer auf deine Liebe und Unterstützung verlassen. Du hast uns immer in unseren Entscheidungen bekräftigt und an das Gelingen unserer Vorhaben geglaubt. Und auch als ich vor 10 Jahren kurzerhand beschloss in die Niederlande zu ziehen, hast du mich in meinen Plänen unterstützt - auch wenn es nicht immer einfach für dich war, dass ich seitdem fast 700 $\mathrm{km}$ entfernt lebte. Ich weiß wie stolz du auf mich bist und ich bin dir unendlich dankbar für deine Liebe. 
